\title{
Anomaly and Anomaly-Free Treatment of QFTs Based on Symmetry-preserving Loop Regularization
}

\author{
Yong-Liang Ma and Yue-Liang Wu \\ Institute of Theoretical Physics, Chinese Academy of Sciences, Beijing 100080, China
}

(Dated: June 4, 2018)

\begin{abstract}
The triangle anomaly in massless and massive QED is investigated by adopting the symmetrypreserving loop regularization method proposed recently in [1]. The method is realized in the initial dimension of theory without modifying the original lagrangian, it preserves symmetries under non-Abelian gauge and Poincare transformations in spite of the existence of two intrinsic mass scales $M_{c}$ and $\mu_{s}$ which actually play the roles of UV- and IR-cut off respectively. The axialvector-vector-vector (AVV) triangle diagrams in massless and massive QED are evaluated explicitly by using the loop regularization. It is shown that when the momentum $k$ of external state is soft with $k^{2} \ll \mu_{s}^{2}, m^{2}$ ( $m$ is the mass of loop fermions) and $M_{c} \rightarrow \infty$, both massless and massive QED become anomaly free. The triangle anomaly is found to appear as quantum corrections in the case that $m^{2}, \mu_{s}^{2} \ll k^{2}$ and $M_{c} \rightarrow \infty$. Especially, it is justified that in the massless QED with $\mu_{s}=0$ and $M_{c} \rightarrow \infty$, the triangle anomaly naturally exists as quantum effects in the axial-vector current when the ambiguity caused by the trace of gamma matrices with $\gamma_{5}$ is eliminated by simply using the definition of $\gamma_{5}$. It is explicitly demonstrated how the Ward identity anomaly of currents depends on the treatment for the trace of gamma matrices, which enables us to make a clarification whether the ambiguity of triangle anomaly is caused by the regularization scheme in the perturbation calculations or by the trace of gamma matrices with $\gamma_{5}$. For comparison, an explicit calculation based on the Pauli-Villars regularization and dimensional regularization is carried out and the possible ambiguities of Ward identity anomalies caused from these two regularization schemes are carefully discussed, which include the ambiguities induced by the treatment of the trace of gamma matrices with $\gamma_{5}$ and the action of the external momentum on the amplitude before the direct calculation of the AVV diagram.
\end{abstract}




\section{Contents}

\section{Introduction}

III. Anomaly and Anomaly-free treatment for massless QED in loop regularization 10

A. Anomalv-free treatment in loop regularization
B. Anomaly of massless QED in loop regularization

IV. Anomalv-free condition in massive QED in loop regularization

A. Ward identities under the condition $\mu^{2} \neq 0$ and $M^{2} \rightarrow \infty$

B. Anomaly under the condition $\mu_{0}^{2}=0$ and $M_{0}^{2} \rightarrow \infty$

V. Anomaly in Pauli-Villars regularization

A. Anomalv of massless QED in Pauli-Villars regularization 25

B. Anomaly of massive QED in Pauli-Villars regularization 27

VI. Anomalv in dimensional regularization

A. Anomalv of massless QED in dimensional regularization 30

B. Anomaly of massive QED in dimensional regularization 35

VII. Clarifications for the long-standing ambiguities in perturbation calculations of triangl

A. Calculation of anomaly with 3.11 in massless QED

1. Calculation of anomalv in loop regularization 37

2. Calculation of anomalv by using Pauli-Villars regularization 41

3. Calculation of anomaly by using dimensional regularization 43

B. Calculation of anomaly by using (3.13) in massless QED 45

1. Calculation of anomalv in the loop regularization 46

2. Calculation of anomaly bv using Pauli-Villars regularization 49

3. Calculation of anomaly by using dimensional regularization 50

C. Calculation of anomaly with relation (3.11) in massive QED 52

1. Calculation of anomaly in the loop regularization 52 
2. Calculation of anomaly by using Pauli-Villars regularization 56

3. Calculation of anomaly by using dimensional regularization 57

D. Calculation of anomaly with relation (3.13) in massive QED 58

1. Calculation of anomalv in the loop regularization 58

2. Calculation of anomaly by using Pauli-Villars regularization 62

3. Calculation of anomaly by using dimensional regularization 63

E. On ambiguities of reducing the triangle diagram by acting external momentum 65

1. The calculation with dimensional regularization 65

2. The calculation with Pauli-Villars regularization 66

$\begin{array}{ll}\text { VIII. Conclusions and Remarks } & 67\end{array}$

$\begin{array}{ll}\text { Acknowledgments } & 70\end{array}$

A. Useful Relations of Feynman Parameter Integrals in Loop Regularization 70

B. Useful Relations in Pauli-Villars Regularization. 72

C. momentum integral in dimensional regualarization 73

$\begin{array}{ll}\text { References } & 73\end{array}$

\section{INTRODUCTION}

Symmetry has played an important role and achieved great triumphs in modern physics especially in elementary particle physics, such as the construction of standard model. Theoretically it is known from the Noether's theorem that if a system is invariant under a continue global transformation, there is a conserved current corresponding to the transformation. Although Noether's theorem is a general conclusion which was verified mathematically, while it is a classical conclusion. In quantum theory, the situation can become different and the Noether's theorem may be violated by quantum corrections, which is usually called quantum anomaly. The importance of anomaly arises from the study of $\pi^{0} \rightarrow 2 \gamma$, the decay of this process is forbidden if the classical symmetry is preserved in quantum case, which is in 
contrast with the experimental data for a large decay rate, this is the so called VeltmanSutherland paradox [2]. It implies that some of the conservation laws corresponding to the symmetries in such a process are violated by the quantum corrections. This is known as the

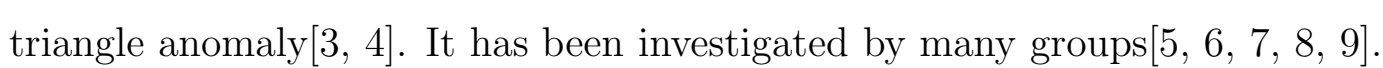

Anomaly as quantum effects becomes significantly important in quantum field theories. In perturbation theory, the anomaly has been calculated by using different regularization schemes, such as Pauli-Villars regularization 10], dimensional regularization 11] and point splitting method 12]. It has also be evaluated in language of path integral[13] and constructed from differential geometry and topological method 14]. In perturbative calculations, one needs to carefully deal with the divergences by choosing appropriate regularization schemes. In the dimensional regularization, it is known to be difficult to define $\gamma_{5}$. In the Pauli-Villars regularization, the field contents of the theory are changed and the non-Abelian gauge invariance cannot well be preserved due to the introduction of massive gauge fields.

In this paper, we shall investigate the anomaly based on the symmetry-preserving loop regularization method developed recently in ref. 11]. Unlike the Pauli-Villars and dimensional regularization schemes, the loop regularization does not change either the contents of lagrangian or the dimension of system. Though its prescription is quite similar to the Pauli-Villars regularization, while the basic concept is quite different. This is because the regularization prescription in the loop regularization is applied to the so-called irreducible loop integrals (ILIs) evaluated from the Feynman loop diagrams rather than to the propagators of additional super-heavy fields made in the Pauli-Villars regularization, which is the reason why loop regularization can satisfy a set of consistency conditions for preserving non-Abelian gauge invariance. In comparison with the dimensional regularization, the loop regularization is carried out in the four dimensional space-time, thus the so-called $\gamma_{5}$ problem in the dimensional regularization does not exist in the loop regularization. In addition, two mass scales are safely introduced in the loop regularization, one is corresponding to the characterizing energy scale $M_{c}$ which can be taken to be infinity $M_{c} \rightarrow \infty$ for underlying renormalizable quantum field theories, another is the sliding energy scale $\mu_{s}$, they actually play the roles of ultraviolet and infrared cut-off scales respectively, so that the loop regularization maintains the well-defined divergent behavior of original integrals when taking $M_{c} \rightarrow \infty$ and $\mu_{s} \rightarrow 0$. In fact, it has been shown that the two mass scales play important roles in understanding the dynamically spontaneous symmetry breaking and the meson 
spectrum [15] for low energy dynamics of QCD.

In the direct calculation of the triangle diagram, the first problem one meets is how to deal with the trace of gamma matrices. There are three possible ways to treat the trace of gamma matrices: (i) directly calculate the trace with the definition of $\gamma_{5}$; (ii) firstly by classifying the Lorentz indices of the two vector currents with another gamma matrix into one group to reduce the number of gamma matrices before the trace; (ii) firstly by classifying the Lorentz indices of one vector current and the axial-vector current with another gamma matrix into one group to reduce the number of gamma matrices before the trace. We shall show that different treatments on the trace of gamma matrices lead to different forms of Ward identity anomaly of the currents. This may be referred as the ambiguity caused by the trace of gamma matrices with $\gamma_{5}$, which is independent of regularization. It is shown that such an ambiguity can simply been eliminated by directly calculating the trace with the definition of $\gamma_{5}$, which enables us to clarify the ambiguity solely arising from the regularization schemes. In all treatments of the gamma matrices, it will be seen that the IR-cut off energy scale $\mu_{s}$ plays a crucial role in understanding anomaly. As either the massless QED or massive QED is a renormalizable quantum field theory, one can always take UV-cut off energy scale $M_{c}$ to be infinity, which does not affect the analysis of anomaly.

For massless QED, it will be demonstrated that the introduction of IR-cut off scale $\mu_{s}$ makes both the vector and axial-vector currents conserved. It is then implies that the loop regularization with $\mu_{s} \neq 0$ ensures Ward identities for both the vector and axial-vector currents. While in the absence of the IR-cut off scale, i.e., $\mu_{s}=0$, we will show that the standard form of axial anomaly is obtained in the treatment where the trace of gamma matrices was evaluated by simply using the definition of $\gamma_{5}$ in four dimensional space-time. In such a treatment, the trace of gamma matrices is unique without ambiguities. For the other treatments of currents, we will see that, in the treatment where the two gamma matrices which carry the Lorentz indices of the vector currents and the gamma matrix between them are classified into one group to reduce the number of gamma matrices, the vector Ward identities are violated and the axial-vector Ward identity is preserved. While in the treatment, where the two gamma matrices with the Lorentz indices of one vector and one axial-vector currents and the gamma between them are classified into one group to reduce the number of gamma matrices, it is found that the Ward identities for the two currents which are grouped become violated while the remaining vector Ward identity is 
kept. It is clear that such ambiguities are caused by the trace of gamma matrices with $\gamma_{5}$. Nevertheless, in both cases, by redefining the physical vector current to be conserved, the anomaly appears in the axial-vector Ward identity with a standard form.

Similarly, for massive QED, the calculation shows that in the treatment where the definition of $\gamma_{5}$ are explicitly used, two vector currents are conserved and the axial-vector Ward identity is in general violated by quantum corrections in the case that $m^{2}, \mu_{s}^{2} \ll k^{2}$ and $M_{c} \rightarrow \infty$. But when the mass $m$ or IR cut-off scale $\mu_{s}$ is sufficiently large in comparison with the momentum of external states, no anomaly will appear in massive QED. In the treatment where the two vector indices are grouped, both the vector Ward identities have anomaly terms and the axial-vector becomes conserved, but both vector and axial-vector currents can be made to be conserved and consequently no anomaly will appear in massive QED when the mass $m$ or IR cut-off scale $\mu_{s}$ is sufficiently large in comparison with the momentum of external states. In the treatment where one vector and the axial-vector currents are grouped, there is anomaly in the grouped vector and axial-vector currents while the other vector current is conserved automatically. Similarly, by a redefinition, two vector currents can be made conserved and anomaly arises in the axial-vector Ward identity. But with the same condition that the mass $m$ or IR cut-off scale $\mu_{s}$ is sufficiently large in comparison with the momentum of external states, the massive QED becomes anomaly free.

From the loop regularization, it is clearly seen that in general the triangle anomaly appears when the external momentum scale $k^{2}$ of axial-vector current becomes much larger than the mass $m$ of loop fermions or the IR cut-off scale $\mu_{s}$. An explicit calculation shows that anomaly terms arise from the convergent integrals as well as the finite parts of the superficially divergent integrals in loop regularization.

The paper is organized as follows: Section II is a brief outline of the loop regularization. In section III, the AVV diagrams in massless QED are calculated explicitly in the loop regularization with $\mu_{s} \neq 0$ and $\mu_{s}=0$. In section $\mathrm{IV}$, we calculate both the AVV and PVV diagrams in massive QED. In section V and VI, we present the calculations based on Pauli-Villars and dimensional regularization respectively with $m \neq 0$ and $m=0$. In section VII, we show how the Ward identity anomaly of currents depends on the treatment for the trace of gamma matrices and thus clarify a unique solution for Ward identity anomaly appearing in the axial-vector current. The possible ambiguities of Ward identity anomaly in the dimensional regularization and Pauli-Villars scheme are carefully investigated. Our 
conclusions and remarks will be given in section VIII. Some useful formulations are listed in Appendix.

\section{A BRIEF OUTLINE OF LOOP REGULARIZATION}

In the quantum field theory, it is inevitable to deal with the infinity problem in the momentum integral. To carry out the integral explicitly, one must first make the infinite integrals be well-defined, which is the so called regularization. Several regularization methods have been proposed, the typical ones include dimensional regularization and Pauli-Villars regularization as well as the most recently proposed symmetry-preserving loop regularization[1] .

To propose a regularization, several elements should be considered, such as the Lorentz invariance, gauge invariance, chiral properties and the behavior of divergence. In the dimensional regularization, the momentum integral of Feynman loops is performed by an analytic continuation of dimensions, it does preserve the Lorentz invariance and gauge invariance when $\gamma_{5}$ is not involved. Once $\gamma_{5}$ is concerned, the dimensional regularization faces problem since $\gamma_{5}$ is an intrinsically four dimensional object. Although a redefinition of $\gamma_{5}$ given in 11] can preserve gauge symmetry at one loop level, while such a redefinition will destroy the gauge symmetry at two loop level[16]. In the Pauli-Villars regularization, the momentum integral is carried out in four dimensions, while the field content is changed by the introduction of heavy massive fields in Pauli-Villars regularization in order to make the integral finite. Although the Abelian gauge symmetry can be preserved, while it is not applicable to the non-Abelian gauge symmetries as the introduction of heavy massive nonabelian gauge fields will destroy gauge symmetry.

It is then of interest to find out a regularization which does not modify the Lagrangian of original theory, and meanwhile preserves both the Lorentz and gauge symmetries, as well as maintains the behavior of divergent integrals by the introduction of intrinsic mass scales. The symmetry-preserving loop regularization[1] has been found to satisfy the above requirements. In the loop regularization, the key concept is the introduction of irreducible loop integrals (ILIs) which are evaluated from Feynman integrals. For instance, at the oneloop level, all Feynman integrals can be evaluated into the following irreducible loop integrals 
(ILIs)

$$
\begin{aligned}
I_{-2 a} & =\int \frac{d^{4} k}{(2 \pi)^{4}} \frac{1}{\left(k^{2}-M^{2}\right)^{2+a}} \\
I_{-2 a \mu \nu} & =\int \frac{d^{4} k}{(2 \pi)^{4}} \frac{k_{\mu} k_{\nu}}{\left(k^{2}-M^{2}\right)^{3+a}}, \quad a=-1,0,1,2, \cdots \\
I_{-2 a \mu \nu \alpha \beta} & =\int \frac{d^{4} k}{(2 \pi)^{4}} \frac{k_{\mu} k_{\nu} k_{\alpha} k_{\beta}}{\left(k^{2}-M^{2}\right)^{2+a}}
\end{aligned}
$$

As shown in [1], to maintain the Lorentz invariance and gauge invariance, the regularized ILIs should satisfy a set of consistent conditions [1]

$$
\begin{aligned}
I_{2 \mu \nu}^{R} & =\frac{1}{2} g_{\mu \nu} I_{2}^{R}, & I_{2 \mu \nu \rho \sigma}^{R}=\frac{1}{8} g_{\{\mu \nu} g_{\rho \sigma\}} I_{2}^{R} \\
I_{0 \mu \nu}^{R} & =\frac{1}{4} g_{\mu \nu} I_{0}^{R}, & I_{0 \mu \nu \rho \sigma}^{R}=\frac{1}{24} g_{\{\mu \nu} g_{\rho \sigma\}} I_{0}^{R} \\
I_{-2 \mu \nu}^{R} & =\frac{1}{6} g_{\mu \nu} I_{-2}^{R}, & I_{-2 \mu \nu \rho \sigma}^{R}=\frac{1}{48} g_{\{\mu \nu} g_{\rho \sigma\}} I_{-2}^{R}
\end{aligned}
$$

where $g_{\{\mu \nu} g_{\rho \sigma\}} \equiv g_{\mu \nu} g_{\rho \sigma}+g_{\mu \rho} g_{\nu \sigma}+g_{\mu \sigma} g_{\nu \rho}$.

The prescription of loop regularization is simple: replacing the integration variable $k^{2}$ and integration measure $\int \frac{d^{4} k}{(2 \pi)^{4}}$ by the regularized ones as [1]

$$
k^{2} \rightarrow\left[k^{2}\right]_{l} \equiv k^{2}-M_{l}^{2}, \quad \int \frac{d^{4} k}{(2 \pi)^{4}} \rightarrow \int\left[\frac{d^{4} k}{(2 \pi)^{4}}\right]_{l} \equiv \lim _{N, M_{i}^{2}} \sum_{l=0}^{N} c_{l}^{N} \int \frac{d^{4} k}{(2 \pi)^{4}}
$$

with conditions

$$
\lim _{N, M_{i}^{2}} \sum_{l=0}^{N} c_{l}^{N}\left(M_{l}^{2}\right)^{n}=0, \quad c_{0}^{N}=1 \quad(i=0,1, \cdots, N \quad \text { and } n=0,1, \cdots)
$$

where $c_{l}^{N}$ is the coefficients determined by the above conditions. The regularized ILIs are then given by [1]

$$
\begin{aligned}
I_{-2 a}^{R} & =\lim _{N, M_{i}^{2}} \sum_{l=0}^{N} c_{l}^{N} \int \frac{d^{4} k}{(2 \pi)^{4}} \frac{1}{\left(k^{2}-M_{l}^{2}\right)^{2+a}} \\
I_{-2 a \mu \nu}^{R} & =\lim _{N, M_{i}^{2}} \sum_{l=0}^{N} c_{l}^{N} \int \frac{d^{4} k}{(2 \pi)^{4}} \frac{k_{\mu} k_{\nu}}{\left(k^{2}-M_{l}^{2}\right)^{3+a}}, \quad a=-1,0,1,2, \cdots \\
I_{-2 a \mu \nu \rho \sigma}^{R} & =\lim _{N, M_{i}^{2}} \sum_{l=0}^{N} c_{l}^{N} \int \frac{d^{4} k}{(2 \pi)^{4}} \frac{k_{\mu} k_{\nu} k_{\rho} k_{\sigma}}{\left(k^{2}-M_{l}^{2}\right)^{2+a}}
\end{aligned}
$$

which can be shown to satisfy the consistency conditions.

An explicit and simple solution of the above equations has been found in [1]

$$
M_{l}^{2}=\mu_{s}^{2}+l M_{R}^{2}, \quad c_{l}^{N}=(-1)^{l} \frac{N !}{(N-l) ! l !}
$$


Here $M_{R}$ is an arbitrary mass scale and $N$ represents the regulator number. It should be noticed that this is different from Pauli-Villars regularization scheme which introduces additional propagators of new fields. Considering the consistent conditions given in (2.2), we only need to evaluate $I_{2}^{R}, I_{0}^{R}$ and $I_{-2}^{R}$. Their explicit forms are given by 1$]$

$$
\begin{aligned}
I_{2}^{R} & =-\frac{i}{16 \pi^{2}}\left\{M_{c}^{2}-\mu^{2}\left[\ln \frac{M_{c}^{2}}{\mu^{2}}-\gamma_{\omega}+1+y_{2}\left(\frac{\mu^{2}}{M_{c}^{2}}\right)\right]\right\} \\
I_{0}^{R} & =\frac{i}{16 \pi^{2}}\left[\ln \frac{M_{c}^{2}}{\mu^{2}}-\gamma_{\omega}+y_{0}\left(\frac{\mu^{2}}{M_{c}^{2}}\right)\right] \\
I_{-2}^{R} & =-\frac{i}{16 \pi^{2}} \frac{1}{2 \mu^{2}}\left[1-y_{-2}\left(\frac{\mu^{2}}{M_{c}^{2}}\right)\right]
\end{aligned}
$$

with $\mu^{2}=\mu_{s}^{2}+M^{2}, \gamma_{w}=\gamma_{E}=0.5772 \cdots$, and

$$
\begin{aligned}
& y_{-2}(x)=1-e^{-x} \\
& y_{0}(x)=\int_{0}^{x} d \sigma \frac{1-e^{-\sigma}}{\sigma}, \quad y_{1}(x)=\frac{e^{-x}-1+x}{x} \\
& y_{2}(x)=y_{0}(x)-y_{1}(x), \quad M_{c}^{2}=\lim _{N, M_{R}} M_{R}^{2} / \ln N
\end{aligned}
$$

Where $\mu_{s}$ sets an IR 'cutoff' at $M^{2}=0$ and $M_{c}$ provides an UV 'cutoff'. More generally speaking, $M_{c}$ and $\mu_{s}$ play the role of characterizing and sliding energy scales respectively. For renormalizable quantum field theories, $M_{c}$ can be taken to be infinity $\left(M_{c} \rightarrow \infty\right)$. $\mu_{s}$ can safely runs to $\mu_{s}=0$ in a theory without infrared divergence. In fact, taking $M_{l} \rightarrow \infty$ (or $\left.M_{R} \rightarrow \infty\right)$ and $\mu_{s}=0$ in the regularization is to recover the initial integral. Also once $M_{R}$ and $N$ are taken to be infinity, the regularized theory becomes independent of regulators. For a detailed description and proof on loop regularization, it is referred to the references in [1].

As an illustration, we make an evaluation for a fermionic loop in massless QED. In massless QED, the electronic loop is given by

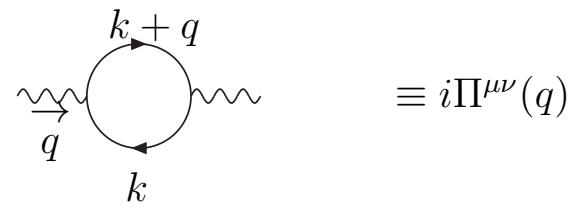

Fig1. Self-energy diagram of photon in quantum electrodynamics

One can write $i \Pi^{\mu \nu}(q)$ explicitly as

$$
i \Pi^{\mu \nu}(q)=(-i e)^{2}(-1) \int \frac{d^{4} k}{(2 \pi)^{4}} \operatorname{tr}\left[\gamma^{\mu} \frac{i}{\not k} \gamma^{\nu} \frac{i}{\not k+\not q}\right]
$$


By using the Feynman parametrization, we can combine the denominators and get the form

$$
i \Pi^{\mu \nu}(q)=-e^{2} \int_{0}^{1} d x \int \frac{d^{4} k}{(2 \pi)^{4}} \operatorname{tr}\left\{\frac{\gamma_{\mu} \not k \gamma_{\nu}(\not 1+\not \not k)}{\left[(k+x q)^{2}-M^{2}\right]^{2}}\right\}
$$

where $M^{2}=-x(1-x) q^{2}$. After making a simple evaluation for Dirac algebra and shifting the integration variable, we have

$$
i \Pi^{\mu \nu}(q)=-4 e^{2} \int_{0}^{1} d x\left[\left(2 I_{2, \mu \nu}-g_{\mu \nu} I_{2}\right)+2 x(1-x)\left(q^{2} g_{\mu \nu}-q_{\mu} q_{\nu}\right) I_{0}\right]
$$

where $I_{2, \mu \nu}, I_{2}$ and $I_{0}$ are defined in (2.1). Note that the shifting of the integration variable is allowed as the loop regularization preserves the translational and Lorentz invariance.

It is seen that the logarithmically divergent integral preserves the gauge symmetry while the quadratically divergent part violates the gauge symmetry. To preserve the gauge symmetry, the regularized quadratically divergent integral should satisfy the relation

$$
2 I_{2, \mu \nu}^{R}-g_{\mu \nu} I_{2}^{R}=0
$$

With the prescription of loop regularization, turning to the Euclidean space, the regularized ILIs are given by

$$
\begin{aligned}
I_{2, \mu \nu}^{R} & =-i \sum_{l=0}^{N} c_{l}^{N} \int \frac{d^{4} k}{(2 \pi)^{2}} \frac{k_{\mu} k_{\nu}}{\left(k^{2}+M_{l}^{2}\right)^{2}} \\
I_{2}^{R} & =-i \sum_{l=0}^{N} c_{l}^{N} \int \frac{d^{4} k}{(2 \pi)^{2}} \frac{1}{\left(k^{2}+M_{l}^{2}\right)} \\
I_{0}^{R} & =i \sum_{l=0}^{N} c_{l}^{N} \int \frac{d^{4} k}{(2 \pi)^{2}} \frac{1}{\left(k^{2}+M_{l}^{2}\right)^{2}}
\end{aligned}
$$

Their explicit forms are giving in eqs. (2.7) and (2.8).

\section{ANOMALY AND ANOMALY-FREE TREATMENT FOR MASSLESS QED IN LOOP REGULARIZATION}

We begin with the massless spinor electrodynamics with the lagrangian

$$
\mathcal{L}=\bar{\psi} \gamma^{\mu}\left(i \partial_{\mu}-e \mathcal{A}_{\mu}\right) \psi
$$

In the following calculation we will neglect the coupling constant $e$. The vector current $V_{\mu}(x)$ and axial-vector current $A_{\mu}(x)$ are defined as

$$
V_{\mu}(x)=\bar{\psi}(x) \gamma_{\mu} \psi(x), \quad A_{\mu}(x)=\bar{\psi}(x) \gamma_{\mu} \gamma_{5} \psi(x)
$$


Classically, the above currents are conserved, that is

$$
\partial^{\mu} V_{\mu}(x)=0, \quad \partial^{\mu} A_{\mu}(x)=0
$$

To investigate the quantum corrections, considering the following Green function

$$
T_{\mu \nu \lambda}^{A V V}(p, q ;(p+q))=\int d^{4} x_{1} d^{4} x_{2} e^{i p x_{1}+i q x_{2}}\left\langle 0\left|T\left[V_{\mu}\left(x_{1}\right) V_{\nu}\left(x_{2}\right) A_{\lambda}(0)\right]\right| 0\right\rangle
$$

The corresponding classical Ward identity (3.3) leads to the following relations

$$
\begin{aligned}
& p^{\mu} T_{\mu \nu \lambda}^{A V V}(p, q ;(p+q))=0 \\
& q^{\nu} T_{\mu \nu \lambda}^{A V V}(p, q ;(p+q))=0 \\
& (p+q)^{\lambda} T_{\mu \nu \lambda}^{A V V}(p, q ;(p+q))=0
\end{aligned}
$$

We now calculate the quantum corrections at one loop level. Diagrammatically, $T^{A V V}$ can be represented as follows with its cross one

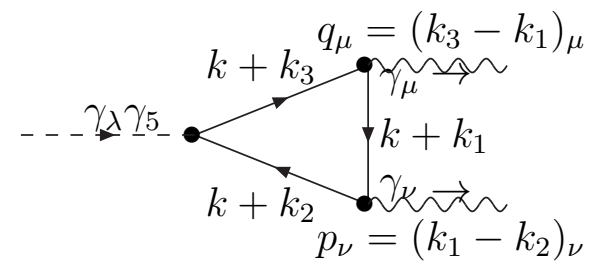

Fig.2. One loop diagrammatical representation of correction to $T^{A V V}$.

One can easily write down the corresponding loop contributions $T_{\lambda \mu \nu}^{(1), A V V}$ from the above diagram

$$
\begin{aligned}
T_{\lambda \mu \nu}^{(1), A V V} & =(-1) \int \frac{d^{4} k}{(2 \pi)^{4}} \operatorname{tr}\left\{\gamma_{\lambda} \gamma_{5} \frac{i}{\left(\not k+\not k_{2}\right)} \gamma_{\nu} \frac{i}{\left(\not k+\not k k_{1}\right)} \gamma_{\mu} \frac{i}{\left(\not k+\not k k_{3}\right)}\right\} \\
& =-i \int \frac{d^{4} k}{(2 \pi)^{4}} \frac{\left(k+k_{2}\right)_{\alpha}\left(k+k_{1}\right)_{\beta}\left(k+k_{3}\right)_{\xi}}{\left(k+k_{2}\right)^{2}\left(k+k_{1}\right)^{2}\left(k+k_{3}\right)^{2}} \operatorname{tr}\left\{\gamma_{5} \gamma_{\lambda} \gamma_{\alpha} \gamma_{\nu} \gamma_{\beta} \gamma_{\mu} \gamma_{\xi}\right\}
\end{aligned}
$$

For the trace of gamma matrix, there are several ways to deal with it. Firstly, by using relation

$$
\gamma_{\mu} \gamma_{\nu} \gamma_{\alpha}=g_{\mu \nu} \gamma_{\alpha}-g_{\mu \alpha} \gamma_{\nu}+g_{\nu \alpha} \gamma_{\mu}-i \epsilon_{\mu \nu \alpha \beta} \gamma_{5} \gamma_{\beta}
$$

we can reduce the number of gamma matrix in the trace to make the trace simpler. Even in the case, there are several ways to classify the gamma matrices. If we select

$$
\gamma_{\nu} \gamma_{\beta} \gamma_{\mu}=g_{\nu \beta} \gamma_{\mu}-g_{\mu \nu} \gamma_{\beta}+g_{\mu \beta} \gamma_{\nu}-i \epsilon_{\nu \beta \mu \sigma} \gamma_{5} \gamma_{\sigma}
$$


we have

$$
\begin{aligned}
\operatorname{tr}\left\{\gamma_{5} \gamma_{\lambda} \gamma_{\alpha} \gamma_{\nu} \gamma_{\beta} \gamma_{\mu} \gamma_{\xi}\right\}= & 4 i\left\{g_{\mu \nu} \epsilon_{\lambda \alpha \beta \xi}-g_{\nu \beta} \epsilon_{\lambda \alpha \mu \xi}-g_{\mu \beta} \epsilon_{\lambda \alpha \nu \xi}\right. \\
& \left.-g_{\lambda \alpha} \epsilon_{\nu \beta \mu \xi}+g_{\alpha \xi} \epsilon_{\nu \beta \mu \lambda}-g_{\lambda \xi} \epsilon_{\nu \beta \mu \alpha}\right\}
\end{aligned}
$$

While if we choose

$$
\gamma_{\lambda} \gamma_{\alpha} \gamma_{\nu}=g_{\lambda \alpha} \gamma_{\nu}-g_{\lambda \nu} \gamma_{\alpha}+g_{\alpha \nu} \gamma_{\lambda}-i \epsilon_{\lambda \alpha \nu \sigma} \gamma_{5} \gamma_{\sigma}
$$

we then arrive at the following result

$$
\begin{aligned}
\operatorname{tr}\left\{\gamma_{5} \gamma_{\lambda} \gamma_{\alpha} \gamma_{\nu} \gamma_{\beta} \gamma_{\mu} \gamma_{\xi}\right\}= & 4 i\left\{g_{\nu \lambda} \epsilon_{\alpha \beta \mu \xi}-g_{\alpha \lambda} \epsilon_{\nu \beta \mu \xi}-g_{\alpha \nu} \epsilon_{\lambda \beta \mu \xi}\right. \\
& \left.-g_{\mu \xi} \epsilon_{\lambda \alpha \nu \beta}+g_{\beta \xi} \epsilon_{\lambda \alpha \nu \mu}-g_{\beta \mu} \epsilon_{\lambda \alpha \nu \xi}\right\}
\end{aligned}
$$

where we have used the trace formula

$$
\operatorname{Tr}\left\{\gamma_{5} \gamma_{\mu} \gamma_{\nu} \gamma_{\alpha} \gamma_{\beta}\right\}=-4 i \epsilon_{\mu \nu \alpha \beta}, \quad \epsilon_{0123}=1
$$

This means that the different classifications of gamma matrices may yield different forms for the tensor structures even they are from the same identity. Namely, although the different forms of the trace should lead to the same result for the given Lorentz indices, while they do not respect to the same symmetry properties of the Lorentz indices for a general case. We will explicitly show their influences on the forms of anomaly in the following sections. This may be referred as the ambiguities caused by the trace of gamma matrices with $\gamma_{5}$, which is independent of any regularization.

As the first step, we shall eliminate such kind of ambiguities before applying for any regularization schemes. For that, we find a unique solution by treating all the three currents symmetrically with adopting the definition of $\gamma_{5}$

$$
\gamma_{5}=\frac{i}{4 !} \epsilon_{\mu \nu \alpha \beta} \gamma_{\mu} \gamma_{\nu} \gamma_{\alpha} \gamma_{\beta}
$$

By repeatedly using the relation $\gamma_{\rho} \gamma_{\sigma}=2 g_{\rho \sigma}-\gamma_{\sigma} \gamma_{\rho}$ to reduce the gamma matrices, eventually we have

$$
\begin{aligned}
\operatorname{Tr}\left\{\gamma_{5} \gamma_{\lambda} \gamma_{\alpha} \gamma_{\nu} \gamma_{\beta} \gamma_{\mu} \gamma_{\xi}\right\}= & \frac{i}{4 !} \epsilon_{\mu \nu \alpha \beta} \operatorname{Tr}\left\{\gamma_{\mu} \gamma_{\nu} \gamma_{\alpha} \gamma_{\beta} \gamma_{\lambda} \gamma_{\alpha} \gamma_{\nu} \gamma_{\beta} \gamma_{\mu} \gamma_{\xi}\right\} \\
= & 4 i\left\{\epsilon_{\lambda \alpha \beta \xi} g_{\mu \nu}-\epsilon_{\lambda \alpha \nu \beta} g_{\mu \xi}+\epsilon_{\lambda \alpha \nu \mu} g_{\beta \xi}-\epsilon_{\lambda \alpha \nu \xi} g_{\mu \beta}-\epsilon_{\lambda \alpha \beta \mu} g_{\nu \xi}\right. \\
& -\epsilon_{\lambda \alpha \mu \xi} g_{\nu \beta}+\epsilon_{\lambda \nu \beta \mu} g_{\alpha \xi}-\epsilon_{\lambda \nu \beta \xi} g_{\alpha \mu}+\epsilon_{\lambda \nu \mu \xi} g_{\alpha \beta}-\epsilon_{\lambda \beta \mu \xi} g_{\alpha \nu} \\
& \left.-\epsilon_{\alpha \nu \beta \mu} g_{\lambda \xi}+\epsilon_{\alpha \nu \beta \xi} g_{\lambda \mu}-\epsilon_{\alpha \nu \mu \xi} g_{\lambda \beta}+\epsilon_{\alpha \beta \mu \xi} g_{\lambda \nu}+\epsilon_{\nu \mu \beta \xi} g_{\lambda \alpha}\right\}
\end{aligned}
$$


which is the most general form respecting all the symmetries of the Lorentz indices and eliminates the ambiguities caused by the trace of gamma matrices with $\gamma_{5}$.

In this section, we shall concentrate on the most general case by treating all the Lorentz indices symmetrically. With the above relation (3.16), after performing the Dirac algebra, the amplitude $T_{\lambda \mu \nu}^{(1), A V V}$ can be written as follows

$$
\begin{aligned}
T_{\lambda \mu \nu}^{(1),\{A V V\}}= & T_{L, \lambda \mu \nu}^{(1),\{A V V\}}+T_{C, \lambda \mu \nu}^{(1),\{A V V\}} \\
T_{L, \lambda \mu \nu}^{(1),\{A V V\}}= & 4 \int \frac{d^{4} k}{(2 \pi)^{4}}\{ \\
& \times\left\{-\epsilon_{\lambda \alpha \nu \beta}\left(k+k_{2}\right)_{\alpha}\left(k+k_{1}\right)_{\beta}\left(k+k_{3}\right)_{\mu}-\epsilon_{\lambda \alpha \nu \rho}\left(k+k_{2}\right)_{\alpha}\left(k+k_{1}\right)_{\mu}\left(k+k_{3}\right)_{\rho}\right. \\
& -\epsilon_{\lambda \alpha \beta \mu}\left(k+k_{2}\right)_{\alpha}\left(k+k_{1}\right)_{\beta}\left(k+k_{3}\right)_{\nu}+\epsilon_{\lambda \alpha \beta \rho} g_{\mu \nu}\left(k+k_{2}\right)_{\alpha}\left(k+k_{1}\right)_{\beta}\left(k+k_{3}\right)_{\rho} \\
& -\epsilon_{\lambda \alpha \mu \rho}\left(k+k_{2}\right)_{\alpha}\left(k+k_{1}\right)_{\nu}\left(k+k_{3}\right)_{\rho}-\epsilon_{\lambda \nu \beta \rho}\left(k+k_{2}\right)_{\mu}\left(k+k_{1}\right)_{\beta}\left(k+k_{3}\right)_{\rho} \\
& -\epsilon_{\lambda \beta \mu \rho}\left(k+k_{2}\right)_{\nu}\left(k+k_{1}\right)_{\beta}\left(k+k_{3}\right)_{\rho}-\epsilon_{\alpha \nu \beta \mu}\left(k+k_{2}\right)_{\alpha}\left(k+k_{1}\right)_{\beta}\left(k+k_{3}\right)_{\lambda} \\
& +\epsilon_{\alpha \nu \beta \rho} g_{\lambda \mu}\left(k+k_{2}\right)_{\alpha}\left(k+k_{1}\right)_{\beta}\left(k+k_{3}\right)_{\rho}-\epsilon_{\alpha \nu \mu \rho}\left(k+k_{2}\right)_{\alpha}\left(k+k_{1}\right)_{\lambda}\left(k+k_{3}\right)_{\rho} \\
& \left.+\epsilon_{\alpha \beta \mu \rho} g_{\lambda \nu}\left(k+k_{2}\right)_{\alpha}\left(k+k_{1}\right)_{\beta}\left(k+k_{3}\right)_{\rho}+\epsilon_{\nu \mu \beta \rho}\left(k+k_{2}\right)_{\lambda}\left(k+k_{1}\right)_{\beta}\left(k+k_{3}\right)_{\rho}\right\} \\
& \times\left[\frac{1}{\left(k+k_{1}\right)^{2}\left(k+k_{2}\right)^{2}\left(k+k_{3}\right)^{2}}\right] \\
& +\frac{\epsilon_{\lambda \alpha \nu \mu}}{2}\left[\frac{\left(k+k_{2}\right)_{\alpha}}{\left(k+k_{2}\right)^{2}\left(k+k_{3}\right)^{2}}+\frac{\left(k+k_{2}\right)_{\alpha}}{\left(k+k_{2}\right)^{2}\left(k+k_{1}\right)^{2}}\right] \\
& +\frac{\epsilon_{\lambda \nu \beta \mu}}{2}\left[\frac{\left(k+k_{1}\right)_{\beta}}{\left(k+k_{1}\right)^{2}\left(k+k_{3}\right)^{2}}+\frac{\left(k+k_{1}\right)_{\beta}}{\left(k+k_{2}\right)^{2}\left(k+k_{1}\right)^{2}}\right] \\
& \left.+\frac{\epsilon_{\lambda \nu \mu \rho}}{2}\left[\frac{\left(k+k_{3}\right)_{\rho}}{\left(k+k_{1}\right)^{2}\left(k+k_{3}\right)^{2}}+\frac{\left(k+k_{3}\right)_{\rho}}{\left(k+k_{2}\right)^{2}\left(k+k_{3}\right)^{2}}\right]\right\} \\
T_{C, \lambda \mu \nu}^{(1),\{A V V\}}= & -2 \epsilon_{\lambda \alpha \nu \mu} \int \frac{d^{4} k}{(2 \pi)^{4}} \frac{\left(k_{3}-k_{1}\right)^{2}\left(k+k_{2}\right)_{\alpha}}{\left(k+k_{1}\right)^{2}\left(k+k_{2}\right)^{2}\left(k+k_{3}\right)^{2}} \\
& -2 \epsilon_{\lambda \nu \beta \mu} \int \frac{d^{4} k}{(2 \pi)^{4}} \frac{\left(k_{3}-k_{2}\right)^{2}\left(k+k_{1}\right)_{\beta}}{\left(k+k_{1}\right)^{2}\left(k+k_{2}\right)^{2}\left(k+k_{3}\right)^{2}} \\
& -2 \epsilon_{\lambda \nu \mu \rho} \int \frac{d^{4} k}{(2 \pi)^{4}} \frac{\left(k_{1}-k_{2}\right)^{2}\left(k+k_{3}\right)_{\rho}}{\left(k+k_{1}\right)^{2}\left(k+k_{2}\right)^{2}\left(k+k_{3}\right)^{2}} \\
& -18)
\end{aligned}
$$

where we have used the identity

$$
\left(k+k_{i}\right) \cdot\left(k+k_{j}\right)=\frac{1}{2}\left(k+k_{i}\right)^{2}+\frac{1}{2}\left(k+k_{j}\right)^{2}-\frac{1}{2}\left(k_{i}-k_{j}\right)^{2}
$$

Applying the loop regularization to the amplitude, as it maintains the translational invariance and respects symmetric integration rules, by shifting the integration variable and making some algebra, the regularized amplitude is found to have the form

$$
T_{\lambda \mu \nu}^{R,(1),\{A V V\}}=T_{0, \lambda \mu \nu}^{R,(1),\{A V V\}}+T_{-2, \lambda \mu \nu}^{R,(1),\{A V V\}}
$$




$$
\begin{aligned}
T_{0, \lambda \mu \nu}^{R,(1),\{A V V\}}= & 2 \int_{0}^{1} d x\left[\epsilon_{\lambda \alpha \nu \mu}\left(k_{2}-k_{1}\right)_{\alpha} I_{0}^{R}\left(x, \mu_{1}\right)+\epsilon_{\lambda \alpha \nu \mu}(2 x-1)\left(k_{3}-k_{2}\right)_{\alpha} I_{0}^{R}\left(x, \mu_{2}\right)\right. \\
& \left.+\epsilon_{\lambda \mu \alpha \nu}\left(k_{3}-k_{1}\right)_{\alpha} I_{0}^{R}\left(x, \mu_{3}\right)\right] \\
& -2 \epsilon_{\lambda \mu \nu \alpha} \int_{0}^{1} d x_{1} \int_{0}^{x_{1}} d x_{2}\left(-2 k_{3}-2 k_{2}+4 k_{1}\right)_{\alpha} I_{0}^{R}\left(x_{i}, \mu\right) \\
T_{-2, \lambda \mu \nu}^{R,(1),\{A V V\}}= & -8 \int_{0}^{1} d x_{1} \int_{0}^{x_{1}} d x_{2} \\
& \times\left\{\epsilon_{\lambda \alpha \nu \beta}\left(-\Delta+k_{2}\right)_{\alpha}\left(-\Delta+k_{1}\right)_{\beta}\left(-\Delta+k_{3}\right)_{\mu}\right. \\
& +\epsilon_{\lambda \alpha \nu \rho}\left(-\Delta+k_{2}\right)_{\alpha}\left(-\Delta+k_{1}\right)_{\mu}\left(-\Delta+k_{3}\right)_{\rho} \\
& +\epsilon_{\lambda \alpha \beta \mu}\left(-\Delta+k_{2}\right)_{\alpha}\left(-\Delta+k_{1}\right)_{\beta}\left(-\Delta+k_{3}\right)_{\nu} \\
& +\epsilon_{\lambda \alpha \mu \rho}\left(-\Delta+k_{2}\right)_{\alpha}\left(-\Delta+k_{1}\right)_{\nu}\left(-\Delta+k_{3}\right)_{\rho} \\
& +\epsilon_{\lambda \nu \beta \rho}\left(-\Delta+k_{2}\right)_{\mu}\left(-\Delta+k_{1}\right)_{\beta}\left(-\Delta+k_{3}\right)_{\rho} \\
& +\epsilon_{\lambda \beta \mu \rho}\left(-\Delta+k_{2}\right)_{\nu}\left(-\Delta+k_{1}\right)_{\beta}\left(-\Delta+k_{3}\right)_{\rho} \\
& +\epsilon_{\alpha \nu \beta \mu}\left(-\Delta+k_{2}\right)_{\alpha}\left(-\Delta+k_{1}\right)_{\beta}\left(-\Delta+k_{3}\right)_{\lambda} \\
& +\epsilon_{\alpha \nu \mu \rho}\left(-\Delta+k_{2}\right)_{\alpha}\left(-\Delta+k_{1}\right)_{\lambda}\left(-\Delta+k_{3}\right)_{\rho} \\
& -\epsilon_{\nu \mu \beta \rho}\left(-\Delta+k_{2}\right)_{\lambda}\left(-\Delta+k_{1}\right)_{\beta}\left(-\Delta+k_{3}\right)_{\rho} \\
& +\frac{\epsilon_{\lambda \alpha \nu \mu}}{2}\left(k_{3}-k_{1}\right)^{2}\left(-\Delta+k_{2}\right)_{\alpha}+\frac{\epsilon_{\lambda \nu \beta \mu}}{2}\left(k_{3}-k_{2}\right)^{2}\left(-\Delta+k_{1}\right)_{\beta} \\
& \left.+\frac{\epsilon_{\lambda \nu \mu \rho}}{2}\left(k_{2}-k_{1}\right)^{2}\left(-\Delta+k_{3}\right)_{\rho}\right\}_{-2}^{R}\left(x_{i}, \mu\right) \\
& -\Delta
\end{aligned}
$$

where $x$ and $x_{i}$ are the Feynman parameters and $\mu^{2}=\mu_{s}^{2}+M^{2}$ and $\mu_{i}^{2}=\mu_{s}^{2}+M_{i}^{2}$. We have introduced the following definitions which will also be used in the calculations below

$$
\begin{aligned}
\Delta & =(1-x) k_{1}+\left(x_{1}-x_{2}\right) k_{2}+x_{2} k_{3} \\
\Delta_{1} & =(1-x) k_{1}+x k_{2} \\
\Delta_{2} & =(1-x) k_{2}+x k_{3} \\
\Delta_{3} & =(1-x) k_{1}+x k_{3} \\
-M^{2} & =\left(x_{1}-x_{2}\right)\left(1-x_{1}\right)\left(k_{1}-k_{2}\right)^{2} \\
& +x_{2}\left(1-x_{1}\right)\left(k_{3}-k_{1}\right)^{2}+x_{2}\left(x_{1}-x_{2}\right)\left(k_{3}-k_{2}\right)^{2} \\
-M_{1}^{2} & =x(1-x)\left(k_{1}-k_{2}\right)^{2} \\
-M_{2}^{2} & =x(1-x)\left(k_{3}-k_{2}\right)^{2} \\
-M_{3}^{2} & =x(1-x)\left(k_{3}-k_{1}\right)^{2}
\end{aligned}
$$

To this step, all the momentum integrals, both the divergent and convergent ones, have been 
carried out explicitly.

Next, we will check all three ward identities. By using the definitions of $\Delta$ and $\Delta_{i}$ and introducing the integrals

$$
\begin{aligned}
& I_{-2,(i j)}\left(\mu_{s}^{2}\right)=\frac{i}{32 \pi^{2}} \int_{0}^{1} d x_{1} \int_{0}^{x_{1}} d x_{2} \frac{x_{2}^{i}\left(x_{1}-x_{2}\right)^{j}}{-M^{2}-\mu_{s}^{2}}\left[1-y_{-2}\left(\frac{\mu_{s}^{2}+M^{2}}{M_{c}^{2}}\right)\right] \\
& I_{0,(00)}\left(\mu_{s}^{2}\right) \equiv \frac{i}{16 \pi^{2}}\left\{\int_{0}^{1} d x_{1} \int_{0}^{x_{1}} d x_{2}\left[\ln \left(\frac{M_{c}^{2}}{M^{2}+\mu_{s}^{2}}\right)-\gamma_{\omega}+y_{0}\left(\frac{\mu_{s}^{2}+M^{2}}{M_{c}^{2}}\right)\right]\right. \\
& \left.-\int_{0}^{1} d x x\left[\ln \left(\frac{M_{c}^{2}}{M_{3}^{2}+\mu_{s}^{2}}\right)-\gamma_{\omega}+y_{0}\left(\frac{\mu_{s}^{2}+M_{3}^{2}}{M_{c}^{2}}\right)\right]\right\} \\
& I_{0,(00)}^{\prime}\left(\mu_{s}^{2}\right) \equiv \frac{i}{16 \pi^{2}}\left\{\int_{0}^{1} d x_{1} \int_{0}^{x_{1}} d x_{2}\left[\ln \left(\frac{M_{c}^{2}}{M^{2}+\mu_{s}^{2}}\right)-\gamma_{\omega}+y_{0}\left(\frac{\mu_{s}^{2}+M^{2}}{M_{c}^{2}}\right)\right]\right. \\
& \left.-\int_{0}^{1} d x x\left[\ln \left(\frac{M_{c}^{2}}{M_{1}^{2}+\mu_{s}^{2}}\right)-\gamma_{\omega}+y_{0}\left(\frac{\mu_{s}^{2}+M_{1}^{2}}{M_{c}^{2}}\right)\right]\right\}
\end{aligned}
$$

we obtain

$$
\begin{aligned}
& \left(k_{1}-k_{2}\right)_{\nu} T_{\lambda \mu \nu}^{R,(1),\{A V V\}}=4 \epsilon_{\lambda \mu \nu \alpha}\left(k_{1}-k_{2}\right)_{\nu}\left(k_{3}-k_{1}\right)_{\alpha} I_{0,(00)} \\
& -8 \epsilon_{\lambda \mu \nu \alpha}\left(k_{1}-k_{2}\right)_{\nu}\left(k_{3}-k_{1}\right)_{\alpha} \\
& \times\left\{\left(k_{1}-k_{2}\right)^{2}\left[2 I_{-2,(01)}-2 I_{-2,(02)}-\frac{1}{2} I_{-2,(00)}\right]\right. \\
& \left.+\left(k_{1}-k_{2}\right) \cdot\left(k_{3}-k_{1}\right)\left[2 I_{-2,(11)}-I_{-2,(10)}\right]\right\} \\
& \left(k_{3}-k_{1}\right)_{\mu} T_{\lambda \mu \nu}^{R,(1),\{A V V\}}=-4 \epsilon_{\lambda \nu \alpha \mu}\left(k_{1}-k_{2}\right)_{\alpha}\left(k_{3}-k_{1}\right)_{\mu} I_{0,(00)}^{\prime} \\
& +8 \epsilon_{\lambda \nu \alpha \mu}\left(k_{1}-k_{2}\right)_{\alpha}\left(k_{3}-k_{1}\right)_{\mu} \\
& \times\left\{\left(k_{1}-k_{2}\right) \cdot\left(k_{3}-k_{1}\right)\left[2 I_{-2,(11)}-I_{-2,(01)}\right]\right. \\
& \left.+\left(k_{3}-k_{1}\right)^{2}\left[2 I_{-2,(10)}-2 I_{-2,(20)}-\frac{1}{2} I_{-2,(00)}\right]\right\} \\
& \left(k_{3}-k_{2}\right)_{\lambda} T_{\lambda \mu \nu}^{R,(1),\{A V V\}}=-4 \epsilon_{\mu \nu \lambda \alpha}\left(k_{3}-k_{1}\right)_{\lambda}\left(k_{1}-k_{2}\right)_{\alpha} I_{0,(00)}^{\prime} \\
& -4 \epsilon_{\mu \nu \lambda \alpha}\left(k_{3}-k_{1}\right)_{\lambda}\left(k_{1}-k_{2}\right)_{\alpha} I_{0,(00)} \\
& -4 \epsilon_{\mu \nu \lambda \alpha}\left(k_{3}-k_{1}\right)_{\lambda}\left(k_{1}-k_{2}\right)_{\alpha} \\
& \times\left\{\left(k_{3}-k_{1}\right)^{2} I_{-2,(00)}+\left(k_{1}-k_{2}\right)^{2} I_{-2,(00)}\right. \\
& \left.+2\left(k_{1}-k_{2}\right) \cdot\left(k_{3}-k_{1}\right)\left[I_{-2,(10)}+I_{-2,(01)}\right]\right\}
\end{aligned}
$$

Note that $I_{0,(00)}$ is the difference between two logarithemically divergent integrals, consequently the above result becomes finite. The explicit expressions and relations for $I_{-2,(i j)}$ and $I_{0,(00)}$ can be read off from App. A. by taking $m^{2}=0$.

In the following subsections, we consider two interesting treatments corresponding to anomaly-free and anomaly in the loop regularization. 


\section{A. Anomaly-free treatment in loop regularization}

By adopting the explicit relations given in App. A, we obtain:

$$
\begin{aligned}
\left(k_{1}-k_{2}\right)_{\nu} T_{\lambda \mu \nu}^{R,(1),\{A V V\}}= & 4 \epsilon_{\lambda \mu \nu \alpha}\left(k_{1}-k_{2}\right)_{\nu}\left(k_{3}-k_{1}\right)_{\alpha} \\
& \times\left\{\frac{i}{16 \pi^{2}}\left[e^{-\mu_{s}^{2} / M_{c}^{2}} \int_{0}^{1} d x_{1} \int_{0}^{x_{1}} d x_{2} e^{-M^{2} / M_{c}^{2}}-\frac{1}{2} Y\left((p+q)^{2}, q^{2}\right)\right]\right. \\
& -q^{2} I_{-2,(10)}-p^{2} I_{-2,(01)}+2 \mu_{s}^{2} I_{-2,(00)} \\
& -2\left\{p^{2} I_{-2,(01)}-\frac{1}{2} p^{2} I_{-2,(00)}\right. \\
& -2\left[\frac{-i}{64 \pi^{2}} e^{-\mu_{s}^{2} / M_{c}^{2}} \int_{0}^{1} d x_{1} \int_{0}^{x_{1}} d x_{2} e^{-M^{2} / M_{c}^{2}}-\frac{\mu_{s}^{2}}{2} I_{-2,(00)}+\frac{q^{2}}{4} I_{-2,(10)}\right. \\
& \left.\left.\left.+\frac{3 p^{2}}{4} I_{-2,(01)}\right]+\left[\frac{-i}{64 \pi^{2}} Y\left((p+q)^{2}, q^{2}\right)+\frac{p^{2}}{2} I_{-2,(00)}\right]\right\}\right\}
\end{aligned}
$$

where the first part in the bracket comes from the difference of two logarithemically divergent integrals and the others come from the convergent integrals. It is easy to check that all terms cancel each other, namely

$$
\left(k_{1}-k_{2}\right)_{\nu} T_{\lambda \mu \nu}^{R,(1),\{A V V\}}=0
$$

Similarly, we have

$$
\left(k_{3}-k_{1}\right)_{\mu} T_{\lambda \mu \nu}^{R,(1),\{A V V\}}=0
$$

Both (3.37) and (3.38) show that, in loop regularization, with the symmetric treatment of all the three currents, the vector currents are conserved.

For the axial-vector current, by using the same method, we can arrive at the following result

$$
\begin{aligned}
\left(k_{3}-k_{2}\right)_{\lambda} T_{\lambda \mu \nu}^{R,(1),\{A V V\}} & =16 \mu_{s}^{2} \epsilon_{\mu \nu \lambda \alpha}\left(k_{1}-k_{2}\right)_{\lambda}\left(k_{3}-k_{1}\right)_{\alpha} I_{-2,(00)}\left(0, \mu_{s}^{2}\right) \\
& +\frac{i}{2 \pi^{2}} \epsilon_{\mu \nu \lambda \alpha}\left(k_{1}-k_{2}\right)_{\lambda}\left(k_{3}-k_{1}\right)_{\alpha} \int_{0}^{1} d x_{1} \int_{0}^{x_{1}} d x_{2} e^{-\left(\mu_{s}^{2}+M^{2}\right) / M_{c}^{2}}
\end{aligned}
$$

Once considering the conditions for massless QED that: $p^{2}=\left(k_{1}-k_{2}\right)^{2}=0, q^{2}=$ $\left(k_{3}-k_{1}\right)^{2}=0,(p+q)^{2}=2 p \cdot q$ is soft with $\mu_{s}^{2} \gg(p+q)^{2}$, we then observe that, to leading order electromagnetism [17], in the loop regularization with $\mu_{s} \neq 0$, the axial-vector current is also conserved for soft initial state, such as pion, with $\mu_{s}^{2} \gg(p+q)^{2}$. More generally, as long as taking the conditions that $p^{2}, q^{2},(p+q)^{2} \ll \mu_{s}^{2} \ll M_{c}^{2} \rightarrow \infty$, the axial-vector current also becomes conserved

$$
\left(k_{3}-k_{2}\right)_{\lambda} T_{\lambda \mu \nu}^{R,(1),\{A V V\}}=0
$$


This is because, under this condition, by considering the behavior of $y_{-2}$ in (2.11), and the definition (3.30), the Ward identity (3.39) becomes

$$
\begin{aligned}
\left(k_{3}-k_{2}\right)_{\lambda} T_{\lambda \mu \nu}^{R,(1),\{A V V\}}= & 16 \mu_{s}^{2} \epsilon_{\mu \nu \lambda \alpha}\left(k_{1}-k_{2}\right)_{\lambda}\left(k_{3}-k_{1}\right)_{\alpha}\left[\int_{0}^{1} d x_{1} \int_{0}^{x_{1}} d x_{2} \frac{-i}{32 \pi^{2}\left(\mu_{s}^{2}+M^{2}\right)}\right. \\
& \left.+O\left(\left(\mu_{s}^{2}+M^{2}\right) / M_{c}^{2}\right)\right] \\
& +\frac{i}{2 \pi^{2}} \epsilon_{\mu \nu \lambda \alpha}\left(k_{1}-k_{2}\right)_{\lambda}\left(k_{3}-k_{1}\right)_{\alpha} \int_{0}^{1} d x_{1} \int_{0}^{x_{1}} d x_{2} e^{-\left(\mu_{s}^{2}+M^{2}\right) / M_{c}^{2}} \\
= & -\frac{i}{4 \pi^{2}} \epsilon_{\mu \nu \lambda \alpha}\left(k_{1}-k_{2}\right)_{\lambda}\left(k_{3}-k_{1}\right)_{\alpha}\left[1+O\left(\frac{p \cdot q}{\mu_{s}^{2}}\right)+O\left(\frac{\mu_{s}^{2}}{M_{c}^{2}}\right)\right] \\
& +\frac{i}{4 \pi^{2}} \epsilon_{\mu \nu \lambda \alpha}\left(k_{1}-k_{2}\right)_{\lambda}\left(k_{3}-k_{1}\right)_{\alpha}\left[1+O\left(\frac{\mu_{s}^{2}}{M_{c}^{2}}\right)+O\left(\frac{p \cdot q}{M_{c}^{2}}\right)\right] \\
= & \frac{i}{4 \pi^{2}} \epsilon_{\mu \nu \lambda \alpha}\left(k_{1}-k_{2}\right)_{\lambda}\left(k_{3}-k_{1}\right)_{\alpha} O\left(\frac{p \cdot q}{\mu_{s}^{2}}, \frac{\mu_{s}^{2}}{M_{c}^{2}}, \frac{p \cdot q}{M_{c}^{2}}\right) \\
= & 0 \quad \text { for } p \cdot q \ll \mu_{s}^{2}, \quad M_{c}^{2} \rightarrow \infty
\end{aligned}
$$

Here we have only considered, similar to the discussions in the literature 17], the leading order contribution and ignored the higher order terms arising from $M^{2} / \mu_{s}^{2}$ in the soft limit that $p^{2}=0, q^{2}=0$ and $(p+q)^{2}=2 p \cdot q \ll \mu_{s}^{2}$.

With the above explicit calculations, we can now arrive at the conclusion that in loop regularization with the introduction of nonzero IR cut-off scale $\mu_{s} \neq 0$ for massless QED, the Ward identities of both vector and axial-vector currents become conserved in the conditions: $p^{2}, q^{2},(p+q)^{2} \ll \mu_{s}^{2} \ll M_{c}^{2} \rightarrow \infty$.

\section{B. Anomaly of massless QED in loop regularization}

We now consider the case that $\mu_{s}=0$. Making a similar evaluation, we obtain the following results

$$
\begin{aligned}
& \left(k_{3}-k_{1}\right)_{\mu} T_{\lambda \mu \nu}^{R,(1),\{A V V\}}=0 \\
& \left(k_{1}-k_{2}\right)_{\nu} T_{\lambda \mu \nu}^{R,(1),\{A V V\}}=0 \\
& \left(k_{3}-k_{2}\right)_{\lambda} T_{\lambda \mu \nu}^{R,(1),\{A V V\}}=\frac{i}{2 \pi^{2}} \epsilon_{\mu \nu \lambda \alpha}\left(k_{1}-k_{2}\right)_{\lambda}\left(k_{3}-k_{1}\right)_{\alpha} \int_{0}^{1} d x_{1} \int_{0}^{x_{1}} d x_{2} e^{-M^{2} / M_{c}^{2}}
\end{aligned}
$$

Taking $M_{c} \rightarrow \infty$, we yield

$$
\begin{aligned}
& \left(k_{3}-k_{1}\right)_{\mu} T_{\lambda \mu \nu}^{R,(1),\{A V V\}}=0 \\
& \left(k_{1}-k_{2}\right)_{\nu} T_{\lambda \mu \nu}^{R,(1),\{A V V\}}=0 \\
& \left(k_{3}-k_{2}\right)_{\lambda} T_{\lambda \mu \nu}^{R,(1),\{A V V\}}=\frac{i}{4 \pi^{2}} \epsilon_{\mu \nu \lambda \alpha}\left(k_{1}-k_{2}\right)_{\lambda}\left(k_{3}-k_{1}\right)_{\alpha}
\end{aligned}
$$


By including the cross diagrams, we finally obtain the Ward identities with anomaly of axial-vector current

$$
\begin{aligned}
& \left(k_{3}-k_{1}\right)_{\mu} T_{\lambda \mu \nu}^{R,\{A V V\}}=0 \\
& \left(k_{1}-k_{2}\right)_{\nu} T_{\lambda \mu \nu}^{R,\{A V V\}}=0 \\
& \left(k_{3}-k_{2}\right)_{\lambda} T_{\lambda \mu \nu}^{R,\{A V V\}}=\frac{i}{2 \pi^{2}} \epsilon_{\mu \nu \alpha \beta}\left(k_{1}-k_{2}\right)_{\alpha}\left(k_{3}-k_{1}\right)_{\beta}
\end{aligned}
$$

Their operator forms are given by

$$
\begin{aligned}
\partial_{\mu} V_{\mu}(x) & =0 \\
\partial_{\mu} A_{\mu}(x) & =\frac{e^{2}}{4 \pi^{2}} \epsilon_{\mu \nu \alpha \beta} \partial^{\alpha} \mathcal{A}^{\mu}(x) \partial^{\beta} \mathcal{A}^{\nu}(x) \\
& =\frac{e^{2}}{8 \pi^{2}} F^{\mu \nu}(x) \widetilde{F}_{\mu \nu}(x)
\end{aligned}
$$

where $\widetilde{F}_{\mu \nu}(x)=\frac{1}{2} \epsilon_{\mu \nu \alpha \beta} F^{\alpha \beta}(x)$ and the coupling constant $e$ was restored. It should be noticed that the coefficient of $\partial^{\alpha} \mathcal{A}^{\mu}(x) \partial^{\beta} \mathcal{A}^{\nu}(x)$ is $e^{2} /\left(4 \pi^{2}\right)$ rather than $e^{2} /\left(2 \pi^{2}\right)$ as the derivative operator can act on both the vector fields $\mathcal{A}_{\mu}(x)$ and $\mathcal{A}_{\nu}(x)$.

In conclusion, it is seen from the above analysis that the IR regulator $\mu_{s}$ plays an important role in understanding the anomaly. Once introducing the IR cut-off scale $\mu_{s}$ in loop regularization so that it satisfies the condition $p^{2}, q^{2},(p+q)^{2} \ll \mu_{s}^{2} \ll M_{c}^{2} \rightarrow \infty$, then both the vector current and axial-vector current are conserved and no anomaly appears. Namely, loop regularization with sufficient large IR cut-off scale $\mu_{s}$ becomes a completely symmetrypreserving regularization. In the absence of the IR cut-off scale $\left(\mu_{s}=0\right)$ for massless QED, the loop regularization leads to the well-known triangle anomaly for the axial-vector current. It is also seen that, in loop regularization, when the trace of gamma matrices are manipulated with the definition of $\gamma_{5}$ directly, the vector currents are automatically preserved, only the axial-vector Ward identity is violated by quantum corrections. That comes to the correct standard form of anomaly.

\section{ANOMALY-FREE CONDITION IN MASSIVE QED IN LOOP REGULAR- IZATION}

Considering the massive spinor electrodynamics with the lagrangian

$$
\mathcal{L}=\bar{\psi} \gamma^{\mu}\left(i \partial_{\mu}-e \mathcal{A}_{\mu}\right) \psi-m \bar{\psi} \psi
$$


Classically, the ward identities of the vector and axial-vector currents are

$$
\partial_{\mu} V_{\mu}(x)=0, \quad \partial_{\mu} A_{\mu}(x)=2 i m P(x)
$$

where

$$
P(x)=\bar{\psi} \gamma_{5} \psi
$$

To calculate the quantum corrections in perturbation theory, besides the amplitude (3.8), one should consider the amplitude

$$
T_{\mu \nu}^{P V V}(p, q ;(p+q))=\int d^{4} x_{1} d^{4} x_{2} e^{i p x_{1}+i q x_{2}}\left\langle 0\left|T\left[V_{\mu}\left(x_{1}\right) V_{\nu}\left(x_{2}\right) P(0)\right]\right| 0\right\rangle
$$

The classical Ward identities (3.3) for massive case become

$$
\begin{aligned}
& p^{\mu} T_{\mu \nu \lambda}^{A V V}(p, q ;(p+q))=0 \\
& q_{\nu} T_{\mu \nu \lambda}^{A V V}(p, q ;(p+q))=0 \\
& (p+q)_{\lambda} T_{\mu \nu \lambda}^{A V V}(p, q ;(p+q))=2 m T_{\mu \nu}^{P V V}
\end{aligned}
$$

The corresponding diagrams and their cross ones are shown as follows
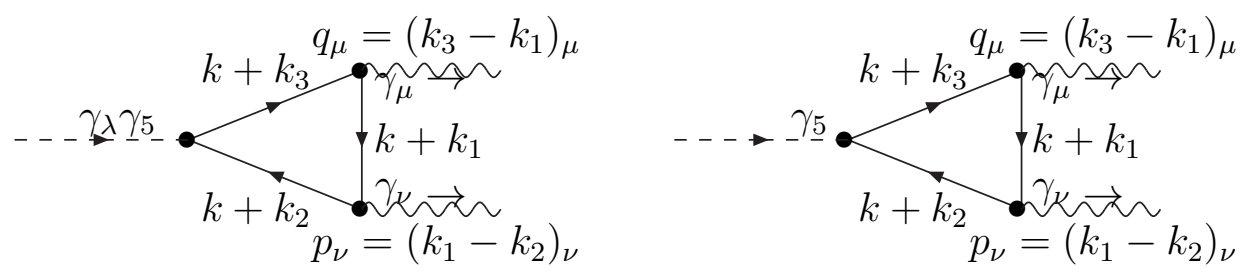

Fig.3. One loop diagrammatical representation of correction to $T^{A V V}$ and $T^{P V V}$.

One can easily write down the corresponding $T_{\lambda \mu \nu}^{A V V}$ and $T_{\lambda \mu \nu}^{P V V}$ from the above diagrams

$$
\begin{aligned}
T_{\lambda \mu \nu}^{(1), A V V} & =(-1) \int \frac{d^{4} k}{(2 \pi)^{4}} \operatorname{tr}\left\{\gamma_{\lambda} \gamma_{5} \frac{i}{\left(\not k+\not k k_{2}\right)-m} \gamma_{\nu} \frac{i}{\left(\not k+\not k k_{1}\right)-m} \gamma_{\mu} \frac{i}{\left(\not k+\not k k_{3}\right)-m}\right\} \\
T_{\mu \nu}^{(1), P V V} & =(-1) \int \frac{d^{4} k}{(2 \pi)^{4}} \operatorname{tr}\left\{\gamma_{5} \frac{i}{(\not k+\not k 2)-m} \gamma_{\nu} \frac{i}{\left(\not k+\not k k_{1}\right)-m} \gamma_{\mu} \frac{i}{\left(\not k+\not k k_{3}\right)-m}\right\}
\end{aligned}
$$

Repeating the same calculations done for massless QED, we yield

$$
\begin{aligned}
T_{\lambda \mu \nu}^{R,(1),\{A V V\}}= & T_{0, \lambda \mu \nu}^{R,(1),\{A V V\}}+T_{-2, \lambda \mu \nu}^{R,(1),\{A V V\}} \\
T_{0, \lambda \mu \nu}^{R,(1),\{A V V\}}= & 2 \int_{0}^{1} d x\left[\epsilon_{\lambda \alpha \nu \mu}\left(k_{2}-k_{1}\right)_{\alpha} I_{0}^{R}\left(x, \mu_{1}\right)+\epsilon_{\lambda \alpha \nu \mu}(2 x-1)\left(k_{3}-k_{2}\right)_{\alpha} I_{0}^{R}\left(x, \mu_{2}\right)\right. \\
& \left.-\epsilon_{\lambda \mu \alpha \nu}\left(k_{3}-k_{1}\right)_{\alpha} I_{0}^{R}\left(x, \mu_{3}\right)\right]
\end{aligned}
$$




$$
\begin{aligned}
T_{-2, \lambda \mu \nu}^{R,(1),\{A V V\}=} & -2 \epsilon_{\lambda \mu \nu \alpha} \int_{0}^{1} d x_{1} \int_{1} \int_{0}^{x_{1}} d x_{2}\left(-2 k_{3}-2 k_{2}+4 k_{1}\right)_{\alpha} I_{0}^{R}\left(x_{i}, \mu\right) \\
& \times\left\{\epsilon_{\lambda \alpha \nu \beta}\left(-\Delta+k_{2}\right)_{\alpha}\left(-\Delta+k_{1}\right)_{\beta}\left(-\Delta+k_{3}\right)_{\mu}\right. \\
& +\epsilon_{\lambda \alpha \nu \rho}\left(-\Delta+k_{2}\right)_{\alpha}\left(-\Delta+k_{1}\right)_{\mu}\left(-\Delta+k_{3}\right)_{\rho} \\
& +\epsilon_{\lambda \alpha \beta \mu}\left(-\Delta+k_{2}\right)_{\alpha}\left(-\Delta+k_{1}\right)_{\beta}\left(-\Delta+k_{3}\right)_{\nu} \\
& +\epsilon_{\lambda \alpha \mu \rho}\left(-\Delta+k_{2}\right)_{\alpha}\left(-\Delta+k_{1}\right)_{\nu}\left(-\Delta+k_{3}\right)_{\rho} \\
& +\epsilon_{\lambda \nu \beta \rho}\left(-\Delta+k_{2}\right)_{\mu}\left(-\Delta+k_{1}\right)_{\beta}\left(-\Delta+k_{3}\right)_{\rho} \\
& +\epsilon_{\lambda \beta \mu \rho}\left(-\Delta+k_{2}\right)_{\nu}\left(-\Delta+k_{1}\right)_{\beta}\left(-\Delta+k_{3}\right)_{\rho} \\
& +\epsilon_{\alpha \nu \beta \mu}\left(-\Delta+k_{2}\right)_{\alpha}\left(-\Delta+k_{1}\right)_{\beta}\left(-\Delta+k_{3}\right)_{\lambda} \\
& +\epsilon_{\alpha \nu \mu \rho}\left(-\Delta+k_{2}\right)_{\alpha}\left(-\Delta+k_{1}\right)_{\lambda}\left(-\Delta+k_{3}\right)_{\rho} \\
& -\epsilon_{\nu \mu \beta \rho}\left(-\Delta+k_{2}\right)_{\lambda}\left(-\Delta+k_{1}\right)_{\beta}\left(-\Delta+k_{3}\right)_{\rho} \\
& +\frac{\epsilon_{\lambda \alpha \nu \mu}}{2}\left(k_{3}-k_{1}\right)^{2}\left(-\Delta+k_{2}\right)_{\alpha}+\frac{\epsilon_{\lambda \nu \beta \mu}}{2}\left(k_{3}-k_{2}\right)^{2}\left(-\Delta+k_{1}\right)_{\beta} \\
& \left.+\frac{\epsilon_{\lambda \nu \mu \rho}}{2}\left(k_{2}-k_{1}\right)^{2}\left(-\Delta+k_{3}\right)_{\rho}\right\}_{-2}^{R}\left(x_{i}, \mu\right) \\
&
\end{aligned}
$$

which has the same form as the massless QED case except the mass of internal fermion is introduced. In above, $x$ and $x_{i}$ are Feynman parameters and

$$
\mu_{i}^{2}=\mu_{s}^{2}+m^{2}+M_{i}^{2}
$$

By using the definitions (3.30)-(3.32), we have

$$
\begin{aligned}
& \left(k_{1}-k_{2}\right)_{\nu} T_{\lambda \mu \nu}^{R,(1),\{A V V\}}=4 \epsilon_{\lambda \mu \nu \alpha}\left(k_{1}-k_{2}\right)_{\nu}\left(k_{3}-k_{1}\right)_{\alpha} I_{0,(00)} \\
& -8 \epsilon_{\lambda \mu \nu \alpha}\left(k_{1}-k_{2}\right)_{\nu}\left(k_{3}-k_{1}\right)_{\alpha} \\
& \times\left\{\left(k_{1}-k_{2}\right)^{2}\left[2 I_{-2,(01)}-2 I_{-2,(02)}-\frac{1}{2} I_{-2,(00)}\right]\right. \\
& \left.+\left(k_{1}-k_{2}\right) \cdot\left(k_{3}-k_{1}\right)\left[2 I_{-2,(11)}-I_{-2,(10)}\right]\right\} \\
& \left(k_{3}-k_{1}\right)_{\mu} T_{\lambda \mu \nu}^{R,(1),\{A V V\}}=-4 \epsilon_{\lambda \nu \alpha \mu}\left(k_{1}-k_{2}\right)_{\alpha}\left(k_{3}-k_{1}\right)_{\mu} I_{0,(00)}^{\prime} \\
& +8 \epsilon_{\lambda \nu \alpha \mu}\left(k_{1}-k_{2}\right)_{\alpha}\left(k_{3}-k_{1}\right)_{\mu} \\
& \times\left\{\left(k_{1}-k_{2}\right) \cdot\left(k_{3}-k_{1}\right)\left[2 I_{-2,(11)}-I_{-2,(01)}\right]\right. \\
& \left.+\left(k_{3}-k_{1}\right)^{2}\left[2 I_{-2,(10)}-2 I_{-2,(20)}-\frac{1}{2} I_{-2,(00)}\right]\right\} \\
& \left(k_{3}-k_{2}\right)_{\lambda} T_{\lambda \mu \nu}^{R,(1),\{A V V\}}=-4 \epsilon_{\mu \nu \lambda \alpha}\left(k_{3}-k_{1}\right)_{\lambda}\left(k_{1}-k_{2}\right)_{\alpha} I_{0,(00)}^{\prime}
\end{aligned}
$$




$$
\begin{aligned}
& -4 \epsilon_{\mu \nu \lambda \alpha}\left(k_{3}-k_{1}\right)_{\lambda}\left(k_{1}-k_{2}\right)_{\alpha} I_{0,(00)} \\
& -4 \epsilon_{\mu \nu \lambda \alpha}\left(k_{3}-k_{1}\right)_{\lambda}\left(k_{1}-k_{2}\right)_{\alpha} \\
& \times\left\{\left(k_{3}-k_{1}\right)^{2} I_{-2,(00)}+\left(k_{1}-k_{2}\right)^{2} I_{-2,(00)}\right. \\
& \left.+2\left(k_{1}-k_{2}\right) \cdot\left(k_{3}-k_{1}\right)\left[I_{-2,(10)}+I_{-2,(01)}\right]\right\}
\end{aligned}
$$

which have the same forms as (3.33)-(3.35) except the mass of internal fermion. In above the quantities $I_{-2,(i j)}$ and $I_{0,(00)}$ are defined in App. A. We now consider two cases with $\mu_{s}^{2} \neq 0$ and $\mu_{s}^{2}=0$.

\section{A. Ward identities under the condition $\mu_{s}^{2} \neq 0$ and $M_{c}^{2} \rightarrow \infty$}

Adopting the relations given in App.A., one can easily write the above Ward identities as

$$
\begin{aligned}
\left(k_{1}-k_{2}\right)_{\nu} T_{\lambda \mu \nu}^{R,(1),\{A V V\}}= & 4 \epsilon_{\lambda \mu \nu \alpha}\left(k_{1}-k_{2}\right)_{\nu}\left(k_{3}-k_{1}\right)_{\alpha} \\
& \times\left\{\frac{i}{16 \pi^{2}}\left[e^{-\left(m^{2}+\mu_{s}^{2}\right) / M_{c}^{2}} \int_{0}^{1} d x_{1} \int_{0}^{x_{1}} d x_{2} e^{-M^{2} / M_{c}^{2}}-\frac{1}{2} Y\left((p+q)^{2}, q^{2}\right)\right]\right. \\
& -q^{2} I_{-2,(10)}-p^{2} I_{-2,(01)}+2\left(\mu_{s}^{2}+m^{2}\right) I_{-2,(00)} \\
& -2\left\{p^{2} I_{-2,(01)}-\frac{1}{2} p^{2} I_{-2,(00)}\right. \\
& -2\left[\frac{-i}{64 \pi^{2}} e^{-\left(m^{2}+\mu_{s}^{2}\right) / M_{c}^{2}} \int_{0}^{1} d x_{1} \int_{0}^{x_{1}} d x_{2} e^{-M^{2} / M_{c}^{2}}-\frac{\mu_{s}^{2}}{2} I_{-2,(00)}\right. \\
+ & \left.\left.\left.\frac{q^{2}}{4} I_{-2,(10)}+\frac{3 p^{2}}{4} I_{-2,(01)}\right]+\left[\frac{-i}{64 \pi^{2}} Y\left((p+q)^{2}, q^{2}\right)+\frac{q^{2}}{2} I_{-2,(00)}\right]\right\}\right\}
\end{aligned}
$$

Again the first part in the bracket comes from the difference of two logarithemically divergent integrals and the others come from the convergent integrals. It is seen that all terms cancel each other, i.e.,

$$
\left(k_{1}-k_{2}\right)_{\nu} T_{\lambda \mu \nu}^{R,(1),\{A V V\}}=0
$$

Similarly, one has

$$
\left(k_{3}-k_{1}\right)_{\mu} T_{\lambda \mu \nu}^{R,(1),\{A V V\}}=0
$$

which shows that for massive QED the situation is same as the massless case, the vector currents remain conserved with the explicit use of the definition of $\gamma_{5}$ in the gamma trace. 
For the axial-vector current, we yield

$$
\begin{aligned}
\left(k_{3}-k_{2}\right)_{\lambda} T_{\lambda \mu \nu}^{R,(1),\{A V V\}}= & -16\left(\mu_{s}^{2}+m^{2}\right) \epsilon_{\mu \nu \lambda \alpha}\left(k_{3}-k_{1}\right)_{\lambda}\left(k_{1}-k_{2}\right)_{\alpha} I_{-2,(00)}\left(\mu_{s}^{2}\right) \\
& -\frac{i}{2 \pi^{2}} \epsilon_{\mu \nu \lambda \alpha}\left(k_{3}-k_{1}\right)_{\lambda}\left(k_{1}-k_{2}\right)_{\alpha} \\
& \times e^{-\left(m^{2}+\mu_{s}^{2}\right) / M_{c}^{2}} \int_{0}^{1} d x_{1} \int_{0}^{x_{1}} d x_{2} e^{-M^{2} / M_{c}^{2}} \\
= & -16 \mu_{s}^{2} \epsilon_{\mu \nu \lambda \alpha}\left(k_{3}-k_{1}\right)_{\lambda}\left(k_{1}-k_{2}\right)_{\alpha} I_{-2,(00)}\left(\mu_{s}^{2}\right) \\
& -\frac{i}{2 \pi^{2}} \epsilon_{\mu \nu \lambda \alpha}\left(k_{3}-k_{1}\right)_{\lambda}\left(k_{1}-k_{2}\right)_{\alpha} \\
& \times e^{-\left(m^{2}+\mu_{s}^{2}\right) / M_{c}^{2}} \int_{0}^{1} d x_{1} \int_{0}^{x_{1}} d x_{2} e^{-M^{2} / M_{c}^{2}} \\
& -16 m^{2} \epsilon_{\mu \nu \lambda \alpha}\left(k_{3}-k_{1}\right)_{\lambda}\left(k_{1}-k_{2}\right)_{\alpha} I_{-2,(00)}\left(\mu_{s}^{2}\right)
\end{aligned}
$$

We now turn to evaluate the PVV diagram. Its loop amplitude is given by

$$
\begin{aligned}
T_{\mu \nu}^{(1), P V V}= & 4 m \int \frac{d^{4} k}{(2 \pi)^{4}} \frac{1}{\left[\left(k+k_{2}\right)^{2}-m^{2}\right]\left[\left(k+k_{1}\right)^{2}-m^{2}\right]\left[\left(k+k_{2}\right)^{2}-m^{2}\right]} \\
& \times\left\{\epsilon_{\alpha \nu \beta \mu}\left(k+k_{2}\right)_{\alpha}\left(k+k_{1}\right)_{\beta}+\epsilon_{\nu \alpha \mu \beta}\left(k+k_{1}\right)_{\alpha}\left(k+k_{3}\right)_{\beta}\right. \\
& \left.+\epsilon_{\alpha \nu \mu \beta}\left(k+k_{2}\right)_{\alpha}\left(k+k_{3}\right)_{\beta}\right\}
\end{aligned}
$$

By using the Feynman parameterization method, it can be rewritten as

$$
\begin{aligned}
T_{\mu \nu}^{(1), P V V}= & 8 m \int_{0}^{1} d x_{1} \int_{0}^{x_{1}} d x_{2} \int \frac{d^{4} k}{(2 \pi)^{4}} \epsilon_{\mu \nu \alpha \beta}\left\{\left\{\left(k+k_{2}\right)_{\alpha}\left(k+k_{1}\right)_{\beta}+\left(k+k_{1}\right)_{\alpha}\left(k+k_{3}\right)_{\beta}\right.\right. \\
& \left.\left.-\left(k+k_{2}\right)_{\alpha}\left(k+k_{3}\right)_{\beta}\right\} \frac{1}{\left[(k+\Delta)^{2}-M^{2}\right]^{3}}\right\}
\end{aligned}
$$

Applying the loop regularization method and making a simple algebraic calculation, we arrive at the following result

$$
\begin{aligned}
T_{\mu \nu}^{R,(1), P V V} & =8 m \sum_{l=0}^{N} c_{l}^{N} \int_{0}^{1} d x_{1} \int_{0}^{x_{1}} d x_{2} \int \frac{d^{4} k}{(2 \pi)^{4}} \epsilon_{\mu \nu \alpha \beta}\left\{\left(k_{1}-k_{2}\right)_{\alpha}\left(k_{3}-k_{1}\right)_{\beta}\right\} \frac{1}{\left[k^{2}-M_{l}^{2}\right]^{3}} \\
& =8 m \epsilon_{\mu \nu \alpha \beta}\left(k_{1}-k_{2}\right)_{\alpha}\left(k_{3}-k_{1}\right)_{\beta} \int_{0}^{1} d x_{1} \int_{0}^{x_{1}} d x_{2} I_{-2}^{R}(\mu) \\
& =8 m \epsilon_{\mu \nu \alpha \beta}\left(k_{1}-k_{2}\right)_{\alpha}\left(k_{3}-k_{1}\right)_{\beta} I_{-2,(00)}
\end{aligned}
$$

with the initial condition $M_{0}^{2}=M^{2}$.

In comparison with eq.(3.39), we obtain the relation between two amplitudes

$$
\begin{aligned}
\left(k_{3}-k_{2}\right)_{\lambda} T_{\lambda \mu \nu}^{R,(1),\{A V V\}}= & -16 \mu_{s}^{2} \epsilon_{\mu \nu \lambda \alpha}\left(k_{3}-k_{1}\right)_{\lambda}\left(k_{1}-k_{2}\right)_{\alpha} I_{-2,(00)}\left(\mu_{s}^{2}\right) \\
& -\frac{i}{2 \pi^{2}} \epsilon_{\mu \nu \lambda \alpha}\left(k_{3}-k_{1}\right)_{\lambda}\left(k_{1}-k_{2}\right)_{\alpha} \\
& \times e^{-\left(m^{2}+\mu_{s}^{2}\right) / M_{c}^{2}} \int_{0}^{1} d x_{1} \int_{0}^{x_{1}} d x_{2} e^{-M^{2} / M_{c}^{2}} \\
& +2 m T_{\mu \nu}^{R,(1), P V V}
\end{aligned}
$$


which implies that the classical axial-vector Ward identity is in general violated by quantum corrections.

Similar to the case in massless QED, if taking the two vector states to be massless, i.e., $p^{2}=q^{2}=0$ and the axial-vector state to be soft with $m^{2}, p \cdot q \ll \mu_{s}^{2} \ll M_{c}^{2} \rightarrow \infty$, as explained in the massless case, we have

$$
\begin{aligned}
& \left(k_{3}-k_{1}\right)_{\mu} T_{\lambda \mu \nu}^{R,(1),\{A V V\}}=0 \\
& \left(k_{1}-k_{2}\right)_{\nu} T_{\lambda \mu \nu}^{R,(1),\{A V V\}}=0 \\
& \left(k_{3}-k_{2}\right)_{\lambda} T_{\lambda \mu \nu}^{R,(1),\{A V V\}}=2 m T_{\mu \nu}^{R,(1), P V V}
\end{aligned}
$$

which indicates that the Ward identities become preserved in this case.

In the case that $\mu_{s} \gg m$, the quantum corrections of the axial-vector Ward identity itself approaches to vanish

$$
\begin{aligned}
\left(k_{3}-k_{2}\right)_{\lambda} T_{\lambda \mu \nu}^{R,(1),\{A V V\}} & =2 m T_{\mu \nu}^{R,(1), P V V} \\
& =-\frac{i}{4 \pi^{2}} \frac{m^{2}}{\mu_{s}^{2}+m^{2}} \epsilon_{\mu \nu \alpha \beta}\left(k_{3}-k_{1}\right)_{\alpha}\left(k_{1}-k_{2}\right)_{\beta} \rightarrow 0
\end{aligned}
$$

Generally speaking, in the limit $p^{2}, q^{2},(p+q)^{2}, m^{2} \ll \mu_{s}^{2} \ll M_{c}^{2} \rightarrow \infty$, both the vector and axial-vector currents become anomaly-free. Namely the Ward identities for both currents are preserved in the quantum corrections. Obviously, to arrive at this conclusion, the IR cut-off scale $\mu_{s}$ plays an important role.

\section{B. Anomaly under the condition $\mu_{s}^{2}=0$ and $M_{c}^{2} \rightarrow \infty$}

In the case that $\mu_{s}^{2}=0$, the relevant Ward identities have the following forms

$$
\begin{aligned}
\left(k_{3}-k_{1}\right)_{\mu} T_{\lambda \mu \nu}^{R,(1),\{A V V\}}= & 0 \\
\left(k_{1}-k_{2}\right)_{\nu} T_{\lambda \mu \nu}^{R,(1),\{A V V\}}= & 0 \\
\left(k_{3}-k_{2}\right)_{\lambda} T_{\lambda \mu \nu}^{R,(1),\{A V V\}=} & -\frac{i}{2 \pi^{2}} \epsilon_{\mu \nu \lambda \alpha}\left(k_{3}-k_{1}\right)_{\lambda}\left(k_{1}-k_{2}\right)_{\alpha} \int_{0}^{1} d x_{1} \int_{0}^{x_{1}} d x_{2} e^{-\left(m^{2}+M^{2}\right) / M_{c}^{2}} \\
& +2 m T_{\mu \nu}^{R,(1), P V V}
\end{aligned}
$$

which indicates that in the loop regularization with $\mu_{s}=0$ the vector Ward identities are preserved, while the axial-vector current is no longer conserved. 
Taking the limit $M_{c}^{2} \rightarrow \infty$, the above results are simplified to be

$$
\begin{aligned}
& \left(k_{3}-k_{1}\right)_{\mu} T_{\lambda \mu \nu}^{R,(1),\{A V V\}}=0 \\
& \left(k_{1}-k_{2}\right)_{\nu} T_{\lambda \mu \nu}^{R,(1),\{A V V\}}=0 \\
& \left(k_{3}-k_{2}\right)_{\lambda} T_{\lambda \mu \nu}^{R,(1),\{A V V\}}=2 m T_{\mu \nu}^{R,(1), P V V}-\frac{i}{4 \pi^{2}} \epsilon_{\mu \nu \lambda \alpha}\left(k_{3}-k_{1}\right)_{\lambda}\left(k_{1}-k_{2}\right)_{\alpha}
\end{aligned}
$$

which arrives at the well-known anomaly in the axial-vector Ward identity.

The above Ward identities can in general be rewritten in form, by considering eq.(4.21), as

$$
\begin{aligned}
\left(k_{3}-k_{1}\right)_{\mu} T_{\lambda \mu \nu}^{R,(1),\{A V V\}} & =0 \\
\left(k_{1}-k_{2}\right)_{\nu} T_{\lambda \mu \nu}^{R,(1),\{A V V\}} & =0 \\
\left(k_{3}-k_{2}\right)_{\lambda} T_{\lambda \mu \nu}^{R,(1),\{A V V\}} & =2 m T_{\mu \nu}^{R,(1), P V V}-\frac{i}{4 \pi^{2}} \epsilon_{\mu \nu \alpha \beta}\left(k_{3}-k_{1}\right)_{\alpha}\left(k_{1}-k_{2}\right)_{\beta} \\
& =(I(m, p, q)-1) \frac{i}{4 \pi^{2}} \epsilon_{\mu \nu \alpha \beta}\left(k_{3}-k_{1}\right)_{\alpha}\left(k_{1}-k_{2}\right)_{\beta}
\end{aligned}
$$

where the integral $I(m, p, q)$ is defined from eq.(4.21) as

$$
I(m, p, q)=\int_{0}^{1} d x_{1} \int_{0}^{x_{1}} d x_{2} \frac{2 m^{2}}{m^{2}+M^{2}}
$$

In the case that the external vector states are massless with on-mass shell conditions: $p^{2}=0$ and $q^{2}=0$ and the axial-vector state is soft with condition $(p+q)^{2}=2 p \cdot q \ll m^{2}$, to leading order electromagnetiam[17], the above integral can simply be performed

$$
I(m, p, q)=\int_{0}^{1} d x_{1} \int_{0}^{x_{1}} d x_{2} \frac{2 m^{2}}{m^{2}+M^{2}}=2 \int_{0}^{1} d x_{1} \int_{0}^{x_{1}} d x_{2}=1
$$

Thus

$$
\left(k_{3}-k_{2}\right)_{\lambda} T_{\lambda \mu \nu}^{R,(1),\{A V V\}}=(I(m, p, q)-1) \frac{i}{4 \pi^{2}} \epsilon_{\mu \nu \alpha \beta}\left(k_{3}-k_{1}\right)_{\alpha}\left(k_{1}-k_{2}\right)_{\beta}=0
$$

Namely the quantum corrections for both vector and axial-vector become vanishing for massive QED when taking the general condition $m^{2} \gg p^{2}, q^{2}, p \cdot q$.

Considering now the alternative case that $p^{2}=0, q^{2}=0$, and $(p+q)^{2} \gg m^{2}$. In this case, the integral $I(m, p, q)$ approaches to zero

$$
I(m, p, q)=\int_{0}^{1} d x_{1} \int_{0}^{x_{1}} d x_{2} \frac{2 m^{2}}{x_{2}\left(x_{2}-x_{1}\right)(p+q)^{2}+m^{2}} \rightarrow 0, \quad(p+q)^{2} \gg m^{2}
$$

which coincides with massless QED at $\mu_{s}=0$. In this case, the axial-vector Ward identity gets anomaly. 
By including the cross diagrams, the Ward identities are given by

$$
\begin{aligned}
& \left(k_{3}-k_{1}\right)_{\mu} T_{\lambda \mu \nu}^{R,\{A V V\}}=0 \\
& \left(k_{1}-k_{2}\right)_{\nu} T_{\lambda \mu \nu}^{R,\{A V V\}}=0 \\
& \left(k_{3}-k_{2}\right)_{\lambda} T_{\lambda \mu \nu}^{R,\{A V V\}}=2 m T_{\mu \nu}^{R, P V V}-\frac{i}{2 \pi^{2}} \epsilon_{\mu \nu \alpha \beta}\left(k_{3}-k_{1}\right)_{\alpha}\left(k_{1}-k_{2}\right)_{\beta}
\end{aligned}
$$

Their operator forms are

$$
\begin{aligned}
\partial_{\mu} V_{\mu}(x) & =0 \\
\partial_{\mu} A_{\mu}(x) & =2 i m P(x)+\frac{e^{2}}{8 \pi^{2}} F^{\mu \nu}(x) \widetilde{F}_{\mu \nu}(x)
\end{aligned}
$$

which is the standard form of triangle anomaly.

It is seen that for massive QED the IR scale $\mu_{s}^{2}$ also plays an important role in understanding the triangle anomaly.

\section{ANOMALY IN PAULI-VILLARS REGULARIZATION}

\section{A. Anomaly of massless QED in Pauli-Villars regularization}

In spinor electrodynamics treated by Pauli-Villars regularization, the fermionic part of the regularized lagrangian is 10$]$

$$
\mathcal{L}_{f}^{R}=\bar{\psi} \gamma^{\mu}\left(i \partial_{\mu}-e \mathcal{A}_{\mu}\right) \psi+\sum_{i=1}^{2} C_{i}\left\{\bar{\psi}_{i} \gamma^{\mu}\left(i \partial_{\mu}-e \mathcal{A}_{\mu}\right) \psi_{i}-m_{i} \bar{\psi}_{i} \psi_{i}\right\}
$$

where $C_{i}$ and mass parameters $m_{i}$ are respectively specified as

$$
\begin{aligned}
& C_{1}=1, \quad C_{2}=-2 \\
& m_{1}^{2}=2 \Lambda^{2}, \quad m_{2}^{2}=\Lambda^{2}
\end{aligned}
$$

Extending the expressions in eqs.(3.20) and (3.21) by adding the mass terms, one arrives at the regularized amplitudes in the Pauli-Villars scheme

$$
\begin{aligned}
T_{\lambda \mu \nu}^{R,(1),\{A V V\}}= & T_{0, \lambda \mu \nu}^{R,(1),\{A V V\}}+T_{-2, \lambda \mu \nu}^{R,(1),\{A V V\}} \\
T_{0, \lambda \mu \nu}^{R,(1),\{A V V\}}= & 2 \sum_{i=0}^{2} C_{i} \int_{0}^{1} d x\left\{\epsilon_{\lambda \alpha \nu \mu}\left(k_{2}-k_{1}\right)_{\alpha} \int \frac{d^{4} k}{(2 \pi)^{4}} \frac{1}{\left[k^{2}-M_{1 i}^{2}\right]^{2}}\right. \\
& +\epsilon_{\lambda \alpha \nu \mu}(2 x-1)\left(k_{3}-k_{2}\right)_{\alpha} \int \frac{d^{4} k}{(2 \pi)^{4}} \frac{1}{\left[k^{2}-M_{2 i}^{2}\right]^{2}}
\end{aligned}
$$




$$
\begin{aligned}
& \left.+\epsilon_{\lambda \mu \alpha \nu}\left(k_{3}-k_{1}\right)_{\alpha} \int \frac{d^{4} k}{(2 \pi)^{4}} \frac{1}{\left[k^{2}-M_{3 i}^{2}\right]^{2}}\right\} \\
& -2 \epsilon_{\lambda \mu \nu \alpha} \int_{0}^{1} d x_{1} \int_{0}^{x_{1}} d x_{2}\left(-2 k_{3}-2 k_{2}+4 k_{1}\right)_{\alpha} \int \frac{d^{4} k}{(2 \pi)^{4}} \frac{k^{2}}{\left[k^{2}-M_{i}^{2}\right]^{3}} \\
& T_{-2, \lambda \mu \nu}^{R,(1),\{A V V\}}=-8 \sum_{i=0}^{2} C_{i} \int_{0}^{1} d x_{1} \int_{0}^{x_{1}} d x_{2} \\
& \times\left\{\epsilon_{\lambda \alpha \nu \beta}\left(-\Delta+k_{2}\right)_{\alpha}\left(-\Delta+k_{1}\right)_{\beta}\left(-\Delta+k_{3}\right)_{\mu}\right. \\
& +\epsilon_{\lambda \alpha \nu \rho}\left(-\Delta+k_{2}\right)_{\alpha}\left(-\Delta+k_{1}\right)_{\mu}\left(-\Delta+k_{3}\right)_{\rho} \\
& +\epsilon_{\lambda \alpha \beta \mu}\left(-\Delta+k_{2}\right)_{\alpha}\left(-\Delta+k_{1}\right)_{\beta}\left(-\Delta+k_{3}\right)_{\nu} \\
& +\epsilon_{\lambda \alpha \mu \rho}\left(-\Delta+k_{2}\right)_{\alpha}\left(-\Delta+k_{1}\right)_{\nu}\left(-\Delta+k_{3}\right)_{\rho} \\
& +\epsilon_{\lambda \nu \beta \rho}\left(-\Delta+k_{2}\right)_{\mu}\left(-\Delta+k_{1}\right)_{\beta}\left(-\Delta+k_{3}\right)_{\rho} \\
& +\epsilon_{\lambda \beta \mu \rho}\left(-\Delta+k_{2}\right)_{\nu}\left(-\Delta+k_{1}\right)_{\beta}\left(-\Delta+k_{3}\right)_{\rho} \\
& +\epsilon_{\alpha \nu \beta \mu}\left(-\Delta+k_{2}\right)_{\alpha}\left(-\Delta+k_{1}\right)_{\beta}\left(-\Delta+k_{3}\right)_{\lambda} \\
& +\epsilon_{\alpha \nu \mu \rho}\left(-\Delta+k_{2}\right)_{\alpha}\left(-\Delta+k_{1}\right)_{\lambda}\left(-\Delta+k_{3}\right)_{\rho} \\
& -\epsilon_{\nu \mu \beta \rho}\left(-\Delta+k_{2}\right)_{\lambda}\left(-\Delta+k_{1}\right)_{\beta}\left(-\Delta+k_{3}\right)_{\rho} \\
& +\frac{\epsilon_{\lambda \alpha \nu \mu}}{2}\left(k_{3}-k_{1}\right)^{2}\left(-\Delta+k_{2}\right)_{\alpha}+\frac{\epsilon_{\lambda \nu \beta \mu}}{2}\left(k_{3}-k_{2}\right)^{2}\left(-\Delta+k_{1}\right)_{\beta} \\
& \left.+\frac{\epsilon_{\lambda \nu \mu \rho}}{2}\left(k_{1}-k_{2}\right)^{2}\left(-\Delta+k_{3}\right)_{\rho}\right\} \int \frac{d^{4} k}{(2 \pi)^{4}} \frac{1}{\left[k^{2}-M_{i}^{2}\right]^{3}}
\end{aligned}
$$

with the initial condition $c_{0}=1$ and $m_{0}=0$ and

$$
\begin{aligned}
-M_{i}^{2} & =\left(x_{1}-x_{2}\right)\left(1-x_{1}\right)\left(k_{2}-k_{1}\right)^{2}+\left(k_{1}-k_{3}\right)^{2} x_{2}\left(1-x_{1}\right) \\
& +\left(k_{3}-k_{2}\right)^{2} x_{2}\left(x_{1}-x_{2}\right)-m_{i}^{2} \\
-M_{1 i}^{2} & =x(1-x)\left(k_{1}-k_{2}\right)^{2}-m_{i}^{2} \\
-M_{2 i}^{2} & =x(1-x)\left(k_{3}-k_{2}\right)^{2}-m_{i}^{2} \\
-M_{3 i}^{2} & =x(1-x)\left(k_{3}-k_{1}\right)^{2}-m_{i}^{2}
\end{aligned}
$$

By adopting the useful relations given in App. B and also in App. A, we have

$$
\begin{aligned}
\left(k_{3}-k_{1}\right)_{\mu} T_{\lambda \mu \nu}^{R,(1),\{A V V\}} & =0 \\
\left(k_{1}-k_{2}\right)_{\nu} T_{\lambda \mu \nu}^{R,(1),\{A V V\}} & =0 \\
\left(k_{3}-k_{2}\right)_{\lambda} T_{\lambda \mu \nu}^{R,(1),\{A V V\}} & =\frac{i}{4 \pi^{2}} \sum_{i=0}^{2} C_{i} \epsilon_{\mu \nu \lambda \alpha}\left(k_{1}-k_{2}\right)_{\lambda}\left(k_{3}-k_{1}\right)_{\alpha}+\sum_{i=0}^{2} C_{i}\left[2 m_{i} T_{\mu \nu}^{(1), P V V}\left(m_{i}\right)\right] \\
& =\sum_{i=0}^{2} C_{i}\left[2 m_{i} T_{\mu \nu}^{(1), P V V}\left(m_{i}\right)\right]
\end{aligned}
$$


which shows that in Pauli-Villars regularization with the general trace relation (3.16) from the definition of $\gamma_{5}$, the vector currents are conserved. Note that such a conclusion is not from the cancellation between the triangle diagram of the original fermion and the regulator super-heavy fermion, it is because both the vector currents in the original triangle and the regulator triangle are conserved separately. While partial cancellation occurs in the axialvector Ward identity.

For axial-vector Ward identity, it needs to further evaluate the term $\sum_{i=0}^{2} C_{i}\left[2 m_{i} T_{\mu \nu}^{(1), P V V}\left(m_{i}\right)\right]$. After some algebra, we have

$$
\begin{aligned}
\sum_{i=0}^{2} C_{i}\left[2 m_{i} T_{\mu \nu}^{(1), P V V}\left(m_{i}\right)\right] & =16 \epsilon_{\mu \nu \alpha \beta}\left(k_{1}-k_{2}\right)_{\alpha}\left(k_{3}-k_{1}\right)_{\beta} \int_{0}^{1} d x_{1} \int_{0}^{x_{1}} d x_{2} \frac{i}{32 \pi^{2}} \sum_{l=1}^{2} C_{i} \frac{m_{i}^{2}}{-M_{i}^{2}} \\
& =\frac{i}{4 \pi^{2}} \epsilon_{\mu \nu \alpha \beta}\left(k_{1}-k_{2}\right)_{\alpha}\left(k_{3}-k_{1}\right)_{\beta}
\end{aligned}
$$

where $m_{1}^{2}$ and $m_{2}^{2}$ have been taken to be infinity large, so that

$$
\frac{m_{i}^{2}}{M_{i}^{2}} \rightarrow 1
$$

By considering the cross diagram, we finally obtain

$$
\left(k_{3}-k_{2}\right)_{\lambda} T_{\lambda \mu \nu}^{R,\{A V V\}}=-\frac{i}{2 \pi^{2}} \epsilon_{\mu \nu \alpha \beta}\left(k_{1}-k_{2}\right)_{\alpha}\left(k_{3}-k_{1}\right)_{\beta}
$$

It is seen that the source of anomaly in Pauli-Villars regularization actually arises from the heavy regulator fermion loops. It is then unclear whether anomaly exists in the original theory or is caused by the regularization scheme.

\section{B. Anomaly of massive QED in Pauli-Villars regularization}

In massive QED, the fermionic part of the regularized lagrangian via Pauli-Villars regularization is $[10]$

$$
\mathcal{L}_{f}^{R}=\bar{\psi} \gamma^{\mu}\left(i \partial_{\mu}-e \mathcal{A}_{\mu}\right) \psi-m \bar{\psi} \psi+\sum_{i=1}^{2} C_{i}\left\{\bar{\psi}_{i} \gamma^{\mu}\left(i \partial_{\mu}-e \mathcal{A}_{\mu}\right) \psi_{i}-m_{i} \bar{\psi}_{i} \psi_{i}\right\}
$$

where $C_{i}$ and mass parameters $m_{i}$ are respectively specified as

$$
\begin{aligned}
& C_{1}=1, \quad C_{2}=-2 \\
& m_{1}^{2}=m^{2}+2 \Lambda^{2}, \quad m_{2}^{2}=m^{2}+\Lambda^{2}
\end{aligned}
$$


With a similar evaluation as the massless case, the regularized amplitudes are given by:

$$
\begin{aligned}
& T_{\lambda \mu \nu}^{R,(1),\{A V V\}}=T_{0, \lambda \mu \nu}^{R,(1),\{A V V\}}+T_{-2, \lambda \mu \nu}^{R,(1),\{A V V\}} \\
& T_{0, \lambda \mu \nu}^{R,(1),\{A V V\}}=2 \sum_{i=0}^{2} C_{i} \int_{0}^{1} d x\left[\epsilon_{\lambda \alpha \mu \nu}\left(k_{2}-k_{1}\right)_{\alpha} \int \frac{d^{4} k}{(2 \pi)^{4}} \frac{1}{\left[k^{2}-M_{1 i}^{2}\right]^{2}}\right. \\
& +\epsilon_{\lambda \alpha \nu \mu}(2 x-1)\left(k_{3}-k_{2}\right)_{\alpha} \int \frac{d^{4} k}{(2 \pi)^{4}} \frac{1}{\left[k^{2}-M_{2 i}^{2}\right]^{2}} \\
& \left.+\epsilon_{\lambda \mu \alpha \nu}\left(k_{3}-k_{1}\right)_{\alpha} \int \frac{d^{4} k}{(2 \pi)^{4}} \frac{1}{\left[k^{2}-M_{3 i}^{2}\right]^{2}}\right] \\
& -2 \epsilon_{\lambda \mu \nu \alpha} \int_{0}^{1} d x_{1} \int_{0}^{x_{1}} d x_{2}\left(-2 k_{3}-2 k_{2}+4 k_{1}\right)_{\alpha} \int \frac{d^{4} k}{(2 \pi)^{4}} \frac{k^{2}}{\left[k^{2}-M_{i}^{2}\right]^{3}} \\
& T_{-2, \lambda \mu \nu}^{R,(1),\{A V V\}}=-8 \sum_{i=0}^{2} C_{i} \int_{0}^{1} d x_{1} \int_{0}^{x_{1}} d x_{2} \\
& \times\left\{\epsilon_{\lambda \alpha \mu \beta}\left(-\Delta+k_{2}\right)_{\alpha}\left(-\Delta+k_{1}\right)_{\beta}\left(-\Delta+k_{3}\right)_{\nu}\right. \\
& +\epsilon_{\lambda \alpha \mu \rho}\left(-\Delta+k_{2}\right)_{\alpha}\left(-\Delta+k_{1}\right)_{\nu}\left(-\Delta+k_{3}\right)_{\rho} \\
& +\epsilon_{\lambda \alpha \beta \nu}\left(-\Delta+k_{2}\right)_{\alpha}\left(-\Delta+k_{1}\right)_{\beta}\left(-\Delta+k_{3}\right)_{\mu} \\
& +\epsilon_{\lambda \alpha \nu \rho}\left(-\Delta+k_{2}\right)_{\alpha}\left(-\Delta+k_{1}\right)_{\mu}\left(-\Delta+k_{3}\right)_{\rho} \\
& +\epsilon_{\lambda \mu \beta \rho}\left(-\Delta+k_{2}\right)_{\nu}\left(-\Delta+k_{1}\right)_{\beta}\left(-\Delta+k_{3}\right)_{\rho} \\
& +\epsilon_{\lambda \beta \nu \rho}\left(-\Delta+k_{2}\right)_{\mu}\left(-\Delta+k_{1}\right)_{\beta}\left(-\Delta+k_{3}\right)_{\rho} \\
& +\epsilon_{\alpha \nu \beta \nu}\left(-\Delta+k_{2}\right)_{\alpha}\left(-\Delta+k_{1}\right)_{\beta}\left(-\Delta+k_{3}\right)_{\lambda} \\
& +\epsilon_{\alpha \mu \nu \rho}\left(-\Delta+k_{2}\right)_{\alpha}\left(-\Delta+k_{1}\right)_{\lambda}\left(-\Delta+k_{3}\right)_{\rho} \\
& -\epsilon_{\alpha \beta \nu \rho}\left(-\Delta+k_{2}\right)_{\alpha}\left(-\Delta+k_{1}\right)_{\beta}\left(-\Delta+k_{3}\right)_{\rho} \\
& -\epsilon_{\mu \nu \beta \rho}\left(-\Delta+k_{2}\right)_{\lambda}\left(-\Delta+k_{1}\right)_{\beta}\left(-\Delta+k_{3}\right)_{\rho} \\
& +\frac{\epsilon_{\lambda \alpha \mu \nu}}{2}\left(k_{3}-k_{1}\right)^{2}\left(-\Delta+k_{2}\right)_{\alpha}+\frac{\epsilon_{\lambda \mu \beta \nu}}{2}\left(k_{3}-k_{2}\right)^{2}\left(-\Delta+k_{1}\right)_{\beta} \\
& \left.+\frac{\epsilon_{\lambda \mu \nu \rho}}{2}\left(k_{2}-k_{1}\right)^{2}\left(-\Delta+k_{3}\right)_{\rho}\right\} \int \frac{d^{4} k}{(2 \pi)^{4}} \frac{1}{\left[k^{2}-M_{i}^{2}\right]^{3}}
\end{aligned}
$$

which has the same form as the massless QED case except the mass of internal fermion is introduced with the initial condition $c_{0}=1$ and $m_{0}=m$.

Again adopting the useful relations presented in App. B and App. A, we obtain the Ward identities as follows

$$
\begin{aligned}
& \left(k_{3}-k_{1}\right)_{\mu} T_{\lambda \mu \nu}^{R,(1),\{A V V\}}=0 \\
& \left(k_{1}-k_{2}\right)_{\nu} T_{\lambda \mu \nu}^{R,(1),\{A V V\}}=0
\end{aligned}
$$




$$
\begin{aligned}
\left(k_{3}-k_{2}\right)_{\lambda} T_{\lambda \mu \nu}^{R,(1),\{A V V\}} & =\frac{i}{4 \pi^{2}} \sum_{i=0}^{2} C_{i} \epsilon_{\mu \nu \lambda \alpha}\left(k_{1}-k_{2}\right)_{\lambda}\left(k_{3}-k_{1}\right)_{\alpha}+\sum_{i=0}^{2} C_{i}\left[2 m_{i} T_{\mu \nu}^{(1), P V V}\left(m_{i}\right)\right] \\
& =\sum_{i=0}^{2} C_{i}\left[2 m_{i} T_{\mu \nu}^{(1), P V V}\left(m_{i}\right)\right]
\end{aligned}
$$

which shows that, in the general relation of the trace of gamma matrices (3.16), the situation is the same as the massless case, the vector currents are conserved automatically, while the axial-vector Ward identity is violated by the quantum corrections.

Here $m_{1}^{2}$ and $m_{2}^{2}$ are the masses of heavy fields and taken to be infinity large. By considering (5.11), we have

$$
\left(k_{3}-k_{2}\right)_{\lambda} T_{\lambda \mu \nu}^{R,(1),\{A V V\}}=2 m T_{\mu \nu}^{(1), P V V}-\frac{i}{4 \pi^{2}} \epsilon_{\mu \nu \alpha \beta}\left(k_{3}-k_{1}\right)_{\alpha}\left(k_{1}-k_{2}\right)_{\beta}
$$

With the same discussions made in the loop regularization for massive QED at $\mu_{s}=0$, the above Ward identity can generally be written as

$$
\left(k_{3}-k_{2}\right)_{\lambda} T_{\lambda \mu \nu}^{(1),\{A V V\}}=(I(m, p, q)-1) \frac{i}{4 \pi^{2}} \epsilon_{\mu \nu \alpha \beta}\left(k_{3}-k_{1}\right)_{\alpha}\left(k_{1}-k_{2}\right)_{\beta}
$$

where $I(m, p, q)$ is define in (4.36).

For two massless vector external states and one soft axial-vector state: $p^{2}=0, q^{2}=0$, $(p+q)^{2}$ is soft, one see from eq.(4.37) that in this case both vector and axial-vector receive no quantum corrections in massive QED.

For the alternative case that $p^{2}=0, q^{2}=0$, and $(p+q)^{2} \gg m^{2}$, thus eq.(4.39) holds, which implies that in this case the axial-vector becomes anomaly. Though Pauli-Villars scheme leads to the same conclusions as the loop regularization at $\mu_{s}=0$, while the anomaly in Pauli-Villars scheme is attribute to the regulator fermion loops rather than the physical fermion loops in the original theory.

\section{ANOMALY IN DIMENSIONAL REGULARIZATION}

Since $\gamma_{5}$ is an intrinsical four dimensional object, to calculate anomaly with dimensional regularization, one can not naively extend it to neither $n \neq 4$ nor non-integer dimensions. In dimensional regularization, $\gamma_{5}$ problem is usually considered by using the definition in $n$ dimensions as follows [11]

$$
\gamma_{5}=i \gamma_{0} \gamma_{1} \gamma_{2} \gamma_{3}
$$


By this definition, then considering the extension of the commutation relation of gamma matrix in $n$ dimension space

$$
\left\{\gamma_{\mu}, \gamma_{\nu}\right\}=2 g_{\mu \nu}
$$

It is not difficult to prove the following commutation relations

$$
\begin{aligned}
& \left\{\gamma_{\mu}^{\|}, \gamma_{5}\right\}=0, \quad \gamma_{\mu}^{\|} \text {is in the first four dimensions } \\
& {\left[\gamma_{\mu}^{\perp}, \gamma_{5}\right]=0, \quad \gamma_{\mu}^{\perp} \text { is in the extended dimensions }}
\end{aligned}
$$

To do the momentum integral with dimensional regularization, only the integration variable is extended to $n$ dimensions while other quantities still live in four dimensions. In the $n$ dimensions, the loop momentum $k$ is decomposed into two parts

$$
k=k_{\|}+k_{\perp}
$$

where $k_{\|}$and $k_{\perp}$ are the components of $k$ in dimensions $0,1,2,3$ and in other $n-4$ dimensions respectively.

\section{A. Anomaly of massless QED in dimensional regularization}

Applying the above considerations to the amplitude defined in (3.8), we then also divide the amplitude into two parts corresponding to $k_{\|}$and $k_{\perp}$

$$
\begin{aligned}
T_{\lambda \mu \nu}^{R,(1), A V V}= & T_{\|, \lambda \mu \nu}^{R,(1), A V V}+T_{\perp, \lambda \mu \nu}^{R,(1), A V V} \\
T_{\|, \lambda \mu \nu}^{R,(1), A V V} \equiv & -i \int \frac{d^{n} k}{(2 \pi)^{n}} \operatorname{tr}\left\{\frac{\gamma_{5} \gamma_{\lambda}\left(\not k_{\|} \| \not k_{2}\right) \gamma_{\nu}\left(\not k_{\|}+\not k_{1}\right) \gamma_{\mu}\left(\not k_{\|}+\not k_{3}\right)}{\left(k+k_{2}\right)^{2}\left(k+k_{1}\right)^{2}\left(k+k_{3}\right)^{2}}\right\} \\
T_{\perp, \lambda \mu \nu}^{R,(1), A V V} \equiv & -4 \epsilon^{\mu \nu \lambda \alpha} \int \frac{d^{n} k}{(2 \pi)^{n}} \frac{\left(k_{\|}+k_{3}\right)_{\alpha} k_{\perp}^{2}}{\left(k+k_{2}\right)^{2}\left(k+k_{1}\right)^{2}\left(k+k_{3}\right)^{2}} \\
& -4 \epsilon^{\mu \nu \lambda \alpha} \int \frac{d^{n} k}{(2 \pi)^{n}} \frac{k_{\perp}^{2}\left(k_{\|}+k_{1}\right)_{\alpha}}{\left(k+k_{1}\right)^{2}\left(k+k_{1}\right)^{2}\left(k+k_{3}\right)^{2}} \\
& \left.-4 \epsilon^{\mu \nu \lambda \alpha} \int \frac{d^{n} k}{(2 \pi)^{n}} \frac{k_{\perp}^{2}\left(k_{\|}+k_{2}\right)_{\alpha}}{\left(k+k_{1}\right)^{2}\left(k+k_{1}\right)^{2}\left(k+k_{3}\right)^{2}}\right\}
\end{aligned}
$$

where we have used eqs.(6.3), (6.4) and (6.5) and only kept the non-zero terms.

For the terms depending on $k_{\perp}^{2}$, by using the Feynman parametrization and App. C., we find that

$$
T_{\perp, \lambda \mu \nu}^{R,(1), A V V}=\frac{1}{3} \frac{i}{4 \pi^{2}} \epsilon_{\mu \nu \lambda \alpha}\left(k_{3}+k_{2}-2 k_{1}\right)_{\alpha}
$$


So that

$$
\begin{aligned}
\left(k_{3}-k_{1}\right)_{\mu} T_{\perp, \lambda \mu \nu}^{R,(1), A V V} & =-\frac{1}{3} \frac{i}{4 \pi^{2}} \epsilon_{\mu \nu \lambda \alpha}\left(k_{3}-k_{1}\right)_{\mu}\left(k_{1}-k_{2}\right)_{\alpha} \\
\left(k_{1}-k_{2}\right)_{\nu} T_{\perp, \lambda \mu \nu}^{R,(1), A V V} & =\frac{1}{3} \frac{i}{4 \pi^{2}} \epsilon_{\mu \nu \lambda \alpha}\left(k_{1}-k_{2}\right)_{\nu}\left(k_{3}-k_{1}\right)_{\alpha} \\
\left(k_{3}-k_{2}\right)_{\lambda} T_{\perp, \lambda \mu \nu}^{R,(1), A V V} & =\frac{2}{3} \frac{i}{4 \pi^{2}} \epsilon_{\mu \nu \lambda \alpha}\left(k_{3}-k_{1}\right)_{\lambda}\left(k_{2}-k_{1}\right)_{\alpha}
\end{aligned}
$$

For the terms independent of $k_{\perp}^{2}$, they are four dimensional objects. By using the relation (3.16) for the trace of gamma matrices, we obtain

$$
\begin{aligned}
& T_{\|, \lambda \mu \nu}^{R,(1),\{A V V\}}=T_{\|, L, \lambda \mu \nu}^{R,(1),\{A V V\}}+T_{\|, C, \lambda \mu \nu}^{R,(1),\{A V V\}} \\
& T_{\|, L, \lambda \mu \nu}^{R,(1),\{A V V\}}=4 \int \frac{d^{4} k}{(2 \pi)^{4}}\left\{\left\{-\epsilon_{\lambda \alpha \nu \beta}\left(k_{\|}+k_{2}\right)_{\alpha}\left(k_{\|}+k_{1}\right)_{\beta}\left(k_{\|}+k_{3}\right)_{\mu}\right.\right. \\
& -\epsilon_{\lambda \alpha \nu \rho}\left(k_{\|}+k_{2}\right)_{\alpha}\left(k_{\|}+k_{1}\right)_{\mu}\left(k_{\|}+k_{3}\right)_{\rho} \\
& -\epsilon_{\lambda \alpha \beta \mu}\left(k_{\|}+k_{2}\right)_{\alpha}\left(k_{\|}+k_{1}\right)_{\beta}\left(k_{\|}+k_{3}\right)_{\nu} \\
& +\epsilon_{\lambda \alpha \beta \rho} g_{\mu \nu}\left(k_{\|}+k_{2}\right)_{\alpha}\left(k_{\|}+k_{1}\right)_{\beta}\left(k_{\|}+k_{3}\right)_{\rho} \\
& -\epsilon_{\lambda \alpha \mu \rho}\left(k_{\|}+k_{2}\right)_{\alpha}\left(k_{\|}+k_{1}\right)_{\nu}\left(k_{\|}+k_{3}\right)_{\rho} \\
& -\epsilon_{\lambda \nu \beta \rho}\left(k_{\|}+k_{2}\right)_{\mu}\left(k_{\|}+k_{1}\right)_{\beta}\left(k_{\|}+k_{3}\right)_{\rho} \\
& -\epsilon_{\lambda \beta \mu \rho}\left(k_{\|}+k_{2}\right)_{\nu}\left(k_{\|}+k_{1}\right)_{\beta}\left(k_{\|}+k_{3}\right)_{\rho} \\
& -\epsilon_{\alpha \nu \beta \mu}\left(k_{\|}+k_{2}\right)_{\alpha}\left(k_{\|}+k_{1}\right)_{\beta}\left(k_{\|}+k_{3}\right)_{\lambda} \\
& +\epsilon_{\alpha \nu \beta \rho} g_{\lambda \mu}\left(k_{\|}+k_{2}\right)_{\alpha}\left(k_{\|}+k_{1}\right)_{\beta}\left(k_{\|}+k_{3}\right)_{\rho} \\
& -\epsilon_{\alpha \nu \mu \rho}\left(k_{\|}+k_{2}\right)_{\alpha}\left(k_{\|}+k_{1}\right)_{\lambda}\left(k_{\|}+k_{3}\right)_{\rho} \\
& +\epsilon_{\alpha \beta \mu \rho} g_{\lambda \nu}\left(k_{\|}+k_{2}\right)_{\alpha}\left(k_{\|}+k_{1}\right)_{\beta}\left(k_{\|}+k_{3}\right)_{\rho} \\
& \left.+\epsilon_{\nu \mu \beta \rho}\left(k_{\|}+k_{2}\right)_{\lambda}\left(k_{\|}+k_{1}\right)_{\beta}\left(k_{\|}+k_{3}\right)_{\rho}\right\} \\
& \times\left[\frac{1}{\left(k+k_{1}\right)^{2}\left(k+k_{2}\right)^{2}\left(k+k_{3}\right)^{2}}\right] \\
& +\frac{\epsilon_{\lambda \alpha \nu \mu}}{2}\left[\frac{\left(k_{\|}+k_{2}\right)_{\alpha}}{\left(k+k_{2}\right)^{2}\left(k+k_{3}\right)^{2}}+\frac{\left(k_{\|}+k_{2}\right)_{\alpha}}{\left(k+k_{2}\right)^{2}\left(k+k_{1}\right)^{2}}\right] \\
& +\frac{\epsilon_{\lambda \nu \beta \mu}}{2}\left[\frac{\left(k_{\|}+k_{1}\right)_{\beta}}{\left(k+k_{1}\right)^{2}\left(k+k_{3}\right)^{2}}+\frac{\left(k_{\|}+k_{1}\right)_{\beta}}{\left(k+k_{2}\right)^{2}\left(k+k_{1}\right)^{2}}\right] \\
& \left.+\frac{\epsilon_{\lambda \nu \mu \rho}}{2}\left[\frac{\left(k_{\|}+k_{3}\right)_{\rho}}{\left(k+k_{1}\right)^{2}\left(k+k_{3}\right)^{2}}+\frac{\left(k_{\|}+k_{3}\right)_{\rho}}{\left(k+k_{2}\right)^{2}\left(k+k_{3}\right)^{2}}\right]\right\} \\
& T_{\|, C, \lambda \mu \nu}^{R,(1),\{A V V\}}=-2 \epsilon_{\lambda \alpha \nu \mu} \int \frac{d^{4} k}{(2 \pi)^{4}} \frac{\left(k_{3}-k_{1}\right)^{2}\left(k_{\|}+k_{2}\right)_{\alpha}}{\left(k+k_{1}\right)^{2}\left(k+k_{2}\right)^{2}\left(k+k_{3}\right)^{2}} \\
& -2 \epsilon_{\lambda \nu \beta \mu} \int \frac{d^{4} k}{(2 \pi)^{4}} \frac{\left(k_{3}-k_{2}\right)^{2}\left(k_{\|}+k_{1}\right)_{\beta}}{\left(k+k_{1}\right)^{2}\left(k+k_{2}\right)^{2}\left(k+k_{3}\right)^{2}}
\end{aligned}
$$




$$
-2 \epsilon_{\lambda \nu \mu \rho} \int \frac{d^{4} k}{(2 \pi)^{4}} \frac{\left(k_{1}-k_{2}\right)^{2}\left(k_{\|}+k_{3}\right)_{\rho}}{\left(k+k_{1}\right)^{2}\left(k+k_{2}\right)^{2}\left(k+k_{3}\right)^{2}}
$$

In the dimensional regularization, we come to the following relation

$$
\begin{aligned}
\int \frac{d^{n} k}{(2 \pi)^{n}} \frac{k_{\| \mu} k_{\| \nu}}{\left[k^{2}-M^{2}\right]^{3}} & =\frac{1}{n} g_{\mu \nu} \int \frac{d^{n} k}{(2 \pi)^{n}} \frac{k_{\|}^{2}}{\left[k^{2}-M^{2}\right]^{3}} \\
& =\frac{1}{n} g_{\mu \nu} \int \frac{d^{n} k}{(2 \pi)^{n}} \frac{k^{2}-k_{\perp}^{2}}{\left[k^{2}-M^{2}\right]^{3}} \\
& =\frac{1}{n} g_{\mu \nu} \int \frac{d^{n} k}{(2 \pi)^{n}}\left\{\frac{1}{\left[k^{2}-M^{2}\right]^{2}}+\frac{M^{2}}{\left[k^{2}-M^{2}\right]^{3}}-\frac{k_{\perp}^{2}}{\left[k^{2}-M^{2}\right]^{3}}\right\} \\
& =\frac{1}{n} g_{\mu \nu} \int \frac{d^{n} k}{(2 \pi)^{n}}\left\{\frac{1}{\left[k^{2}-M^{2}\right]^{2}}\right\}
\end{aligned}
$$

Denoting the infinitesimal constant $\varepsilon$ in the dimensional regularization as $\varepsilon=4-n$, we have

$$
\begin{aligned}
\int \frac{d^{n} k}{(2 \pi)^{n}} \frac{k_{\| \mu} k_{\| \nu}}{\left[k^{2}-M^{2}\right]^{3}} & =\frac{1}{4-\varepsilon} g_{\mu \nu} \int \frac{d^{n} k}{(2 \pi)^{n}}\left\{\frac{1}{\left[k^{2}-M^{2}\right]^{2}}\right\} \\
& =\frac{1}{4} g_{\mu \nu} \int \frac{d^{n} k}{(2 \pi)^{n}}\left\{\frac{1}{\left[k^{2}-M^{2}\right]^{2}}\right\}+\frac{\varepsilon}{16} g_{\mu \nu} \int \frac{d^{n} k}{(2 \pi)^{n}}\left\{\frac{1}{\left[k^{2}-M^{2}\right]^{2}}\right\} \\
& =\frac{1}{4} g_{\mu \nu} \int \frac{d^{n} k}{(2 \pi)^{n}}\left\{\frac{1}{\left[k^{2}-M^{2}\right]^{2}}\right\}+\frac{\varepsilon}{16} g_{\mu \nu} \frac{i}{16 \pi^{2}}\left\{\frac{2}{\varepsilon}+\cdots\right\} \\
& =\frac{1}{4} g_{\mu \nu} \int \frac{d^{n} k}{(2 \pi)^{n}}\left\{\frac{1}{\left[k^{2}-M^{2}\right]^{2}}\right\}+\frac{1}{8} g_{\mu \nu} \frac{i}{16 \pi^{2}}+\mathcal{O}(\varepsilon)
\end{aligned}
$$

We will not consider the last term in the following calculation since it will vanish when one sets $\varepsilon \rightarrow 0$ which is required by the dimensional regularization.

After Feynman paramerization and some algebra, the parallel momentum part of the regularized amplitude $T_{\|, \lambda \mu \nu}^{R,(1),\{A V V\}}$ can be written as:

$$
\begin{aligned}
T_{\|, \lambda \mu \nu}^{R,(1),\{A V V\}}= & T_{\|, 0, \lambda \mu \nu}^{R,(1),\{A V V\}}+T_{\|,-2, \lambda \mu \nu}^{R,(1),\{A V V\}} \\
T_{\|, 0, \lambda \mu \nu}^{R,(1),\{A V V\}}= & 2 \int_{0}^{1} d x\left\{\epsilon_{\lambda \alpha \nu \mu}\left(k_{2}-k_{1}\right)_{\alpha} \frac{i}{16 \pi^{2}}\left[-\ln M_{1}^{2}\right]\right. \\
& +\epsilon_{\lambda \alpha \nu \mu}(2 x-1)\left(k_{3}-k_{2}\right)_{\alpha} \frac{i}{16 \pi^{2}}\left[-\ln M_{2}^{2}\right] \\
& \left.+\epsilon_{\lambda \mu \alpha \nu}\left(k_{3}-k_{1}\right)_{\alpha} \frac{i}{16 \pi^{2}}\left[-\ln M_{3}^{2}\right]\right\} \\
& -2 \epsilon_{\lambda \mu \nu \alpha}\left(-2 k_{3}-2 k_{2}+4 k_{1}\right)_{\alpha} \int_{0}^{1} d x_{1} \int_{0}^{x_{1}} d x_{2} \frac{i}{16 \pi^{2}}\left[-\ln M^{2}\right] \\
T_{\|,-2, \lambda \mu \nu}^{R,(1),\{A V V\}}= & -8 \int_{0}^{1} d x_{1} \int_{0}^{x_{1}} d x_{2} \\
& \times\left\{\epsilon_{\lambda \alpha \nu \beta}\left(-\Delta+k_{2}\right)_{\alpha}\left(-\Delta+k_{1}\right)_{\beta}\left(-\Delta+k_{3}\right)_{\mu}\right.
\end{aligned}
$$




$$
\begin{aligned}
& +\epsilon_{\lambda \alpha \nu \rho}\left(-\Delta+k_{2}\right)_{\alpha}\left(-\Delta+k_{1}\right)_{\mu}\left(-\Delta+k_{3}\right)_{\rho} \\
& +\epsilon_{\lambda \alpha \beta \mu}\left(-\Delta+k_{2}\right)_{\alpha}\left(-\Delta+k_{1}\right)_{\beta}\left(-\Delta+k_{3}\right)_{\nu} \\
& +\epsilon_{\lambda \alpha \mu \rho}\left(-\Delta+k_{2}\right)_{\alpha}\left(-\Delta+k_{1}\right)_{\nu}\left(-\Delta+k_{3}\right)_{\rho} \\
& +\epsilon_{\lambda \nu \beta \rho}\left(-\Delta+k_{2}\right)_{\mu}\left(-\Delta+k_{1}\right)_{\beta}\left(-\Delta+k_{3}\right)_{\rho} \\
& +\epsilon_{\lambda \beta \mu \rho}\left(-\Delta+k_{2}\right)_{\nu}\left(-\Delta+k_{1}\right)_{\beta}\left(-\Delta+k_{3}\right)_{\rho} \\
& +\epsilon_{\alpha \nu \beta \mu}\left(-\Delta+k_{2}\right)_{\alpha}\left(-\Delta+k_{1}\right)_{\beta}\left(-\Delta+k_{3}\right)_{\lambda} \\
& +\epsilon_{\alpha \nu \mu \rho}\left(-\Delta+k_{2}\right)_{\alpha}\left(-\Delta+k_{1}\right)_{\lambda}\left(-\Delta+k_{3}\right)_{\rho} \\
& -\epsilon_{\nu \mu \beta \rho}\left(-\Delta+k_{2}\right)_{\lambda}\left(-\Delta+k_{1}\right)_{\beta}\left(-\Delta+k_{3}\right)_{\rho} \\
& +\frac{\epsilon_{\lambda \alpha \nu \mu}}{2}\left(k_{3}-k_{1}\right)^{2}\left(-\Delta+k_{2}\right)_{\alpha}+\frac{\epsilon_{\lambda \nu \beta \mu}}{2}\left(k_{3}-k_{2}\right)^{2}\left(-\Delta+k_{1}\right)_{\beta} \\
& \left.+\frac{\epsilon_{\lambda \nu \mu \rho}}{2}\left(k_{2}-k_{1}\right)^{2}\left(-\Delta+k_{3}\right)_{\rho}\right\} \frac{-i}{32 \pi^{2}} \frac{1}{M^{2}} \\
& -\frac{i}{16 \pi^{2}} \epsilon_{\lambda \mu \nu \alpha}\left(-k_{3}-k_{2}+2 k_{1}\right)_{\alpha}
\end{aligned}
$$

Here $T_{\|, 0, \lambda \mu \nu}^{(1), A V V}$ and $T_{\|,-2, \lambda \mu \nu}^{R,(1), A V V}$ are corresponding to the logarithemic and convergent terms in (6.13) and (6.14). $x$ and $x_{i}$ are Feynman parameters. The last term in above equation comes from the second term of (6.16).

Using the relations given in App. A. with $\mu_{s}^{2}=0$ and $M_{c}^{2} \rightarrow \infty$, we find that the contributions from the $k_{\|}$have the following forms

$$
\begin{aligned}
\left(k_{3}-k_{1}\right)_{\mu} T_{\|, \lambda \mu \nu}^{R,(1),\{A V V\}} & =-\frac{1}{4} \frac{i}{4 \pi^{2}} \epsilon_{\lambda \mu \nu \alpha}\left(k_{3}-k_{1}\right)_{\mu}\left(k_{1}-k_{2}\right)_{\alpha} \\
\left(k_{1}-k_{2}\right)_{\nu} T_{\|, \lambda \mu \nu}^{R,(1),\{A V V\}} & =\frac{1}{4} \frac{i}{4 \pi^{2}} \epsilon_{\lambda \mu \nu \alpha}\left(k_{1}-k_{2}\right)_{\nu}\left(k_{3}-k_{1}\right)_{\alpha} \\
\left(k_{3}-k_{2}\right)_{\lambda} T_{\|, \lambda \mu \nu}^{R,(1),\{A V V\}} & =\frac{3}{2} \frac{i}{4 \pi^{2}} \epsilon_{\mu \nu \lambda \alpha}\left(k_{1}-k_{2}\right)_{\lambda}\left(k_{3}-k_{1}\right)_{\alpha}
\end{aligned}
$$

Eventually, we yield by combining the two parts the following results

$$
\begin{aligned}
\left(k_{3}-k_{1}\right)_{\mu} T_{\lambda \mu \nu}^{R,(1),\{A V V\}} & =-\frac{7}{12} \frac{i}{4 \pi^{2}} \epsilon_{\mu \nu \lambda \alpha}\left(k_{3}-k_{1}\right)_{\mu}\left(k_{1}-k_{2}\right)_{\alpha} \\
\left(k_{1}-k_{2}\right)_{\nu} T_{\lambda \mu \nu}^{R,(1),\{A V V\}} & =\frac{7}{12} \frac{i}{4 \pi^{2}} \epsilon_{\mu \nu \lambda \alpha}\left(k_{1}-k_{2}\right)_{\nu}\left(k_{3}-k_{1}\right)_{\alpha} \\
\left(k_{3}-k_{2}\right)_{\lambda} T_{\lambda \mu \nu}^{R,(1),\{A V V\}} & =\frac{26}{12} \frac{i}{4 \pi^{2}} \epsilon_{\mu \nu \lambda \beta}\left(k_{1}-k_{2}\right)_{\lambda}\left(k_{3}-k_{1}\right)_{\beta}
\end{aligned}
$$

Including the cross diagrams, we arrive at the final results

$$
\left(k_{3}-k_{1}\right)_{\mu} T_{\lambda \mu \nu}^{R,\{A V V\}}=-\frac{7}{6} \frac{i}{4 \pi^{2}} \epsilon_{\nu \lambda \alpha \beta}\left(k_{1}-k_{2}\right)_{\alpha}\left(k_{3}-k_{1}\right)_{\beta}
$$




$$
\begin{aligned}
\left(k_{1}-k_{2}\right)_{\nu} T_{\lambda \mu \nu}^{R,\{A V V\}} & =\frac{7}{6} \frac{i}{4 \pi^{2}} \epsilon_{\mu \lambda \alpha \beta}\left(k_{1}-k_{2}\right)_{\alpha}\left(k_{3}-k_{1}\right)_{\beta} \\
\left(k_{3}-k_{2}\right)_{\lambda} T_{\lambda \mu \nu}^{R,\{A V V\}} & =\frac{26}{6} \frac{i}{4 \pi^{2}} \epsilon_{\mu \nu \lambda \beta}\left(k_{1}-k_{2}\right)_{\lambda}\left(k_{3}-k_{1}\right)_{\beta}
\end{aligned}
$$

Here both the vector Ward identity and axial-vector identity are violated by quantum anomaly. To keep the vector current be conserved, one can simply make the redefinition for the amplitudes as follows

$$
\widetilde{T}_{\lambda \mu \nu}^{(1),\{A V V\}}\left(k_{3}-k_{1}, k_{1}-k_{2}\right)=T_{\lambda \mu \nu}^{(1),\{A V V\}}\left(k_{3}-k_{1}, k_{1}-k_{2}\right)-T_{\lambda \mu \nu}^{(1),\{A V V\}}(0)
$$

with

$$
T_{\lambda \mu \nu}^{(1),\{A V V\}}(0)=\frac{7}{6} \frac{i}{4 \pi^{2}} \epsilon_{\mu \nu \lambda \alpha}\left[\left(k_{3}-k_{1}\right)_{\alpha}-\left(k_{1}-k_{2}\right)_{\alpha}\right]
$$

As a consequence, the Ward identities of the redefined amplitudes have the standard form of triangle anomaly appearing only in the axial-vector current

$$
\begin{aligned}
& \left(k_{3}-k_{1}\right)_{\mu} \widetilde{T}_{\lambda \mu \nu}^{\{A V V\}}=0 \\
& \left(k_{1}-k_{2}\right)_{\nu} \widetilde{T}_{\lambda \mu \nu}^{\{A V V\}}=0 \\
& \left(k_{3}-k_{2}\right)_{\lambda} \widetilde{T}_{\lambda \mu \nu}^{\{A V V\}}=\frac{i}{2 \pi^{2}} \epsilon_{\mu \nu \lambda \alpha}\left(k_{1}-k_{2}\right)_{\lambda}\left(k_{3}-k_{1}\right)_{\alpha}
\end{aligned}
$$

From the above explicit calculations in the dimensional regularization, we see that, unlike in the loop regularization, the divergences of both the vector and axial-vector currents are violated by quantum corrections. The quantum corrections depend on both the original four dimensions and the extended dimensions. While as shown in[11], if one multiplies the external momentum of the axial-vector current on the AVV diagram before evaluating the integrals, the final results only depend on the extended dimensions and consequently only the axial-vector Ward identity is violated by the anomaly. This implies that in the dimensional regularization the anomaly of vector and axial-vector currents due to quantum loop corrections depends on the procedure of operation although the final anomaly, when normalizing to the conserved vector current, has the same result of the standard form. We shall further discuss the possible ambiguities arising from the dimensional regularization. 


\section{B. Anomaly of massive QED in dimensional regularization}

In the massive QED, with the same reasoning as the massless case, we can write the regularized parallel part of the amplitude as

$$
\begin{aligned}
& T_{\|, \lambda \mu \nu}^{R,(1),\{A V V\}}=T_{\|, 0, \lambda \mu \nu}^{R(1),\{A V V\}}+T_{\|,-2, \lambda \mu \nu}^{R,(1),\{A V V\}} \\
& T_{\|, 0, \lambda \mu \nu}^{R,(1),\{A V V\}}=2 \int_{0}^{1} d x\left\{\epsilon_{\lambda \alpha \mu \nu}\left(k_{2}-k_{1}\right)_{\alpha} \frac{i}{16 \pi^{2}}\left[-\ln \left(M_{1}^{2}+m^{2}\right)\right]\right. \\
& +\epsilon_{\lambda \alpha \nu \mu}(2 x-1)\left(k_{3}-k_{2}\right)_{\alpha} \frac{i}{16 \pi^{2}}\left[-\ln \left(M_{2}^{2}+m^{2}\right)\right] \\
& \left.+\epsilon_{\lambda \mu \alpha \nu}\left(k_{3}-k_{1}\right)_{\alpha} \frac{i}{16 \pi^{2}}\left[-\ln \left(M_{3}^{2}+m^{2}\right)\right]\right\} \\
& -2 \epsilon_{\lambda \mu \nu \alpha} \int_{0}^{1} d x_{1} \int_{0}^{x_{1}} d x_{2}\left(-2 k_{3}-2 k_{2}+4 k_{1}\right)_{\alpha} \frac{i}{16 \pi^{2}}\left[-\ln \left(M^{2}+m^{2}\right)\right] \\
& T_{\|,-2, \lambda \mu \nu}^{R,(1),\{A V V\}}=-8 \int_{0}^{1} d x_{1} \int_{0}^{x_{1}} d x_{2} \\
& \times\left\{\epsilon_{\lambda \alpha \mu \beta}\left(-\Delta+k_{2}\right)_{\alpha}\left(-\Delta+k_{1}\right)_{\beta}\left(-\Delta+k_{3}\right)_{\nu}\right. \\
& +\epsilon_{\lambda \alpha \mu \rho}\left(-\Delta+k_{2}\right)_{\alpha}\left(-\Delta+k_{1}\right)_{\nu}\left(-\Delta+k_{3}\right)_{\rho} \\
& +\epsilon_{\lambda \alpha \beta \nu}\left(-\Delta+k_{2}\right)_{\alpha}\left(-\Delta+k_{1}\right)_{\beta}\left(-\Delta+k_{3}\right)_{\mu} \\
& +\epsilon_{\lambda \alpha \nu \rho}\left(-\Delta+k_{2}\right)_{\alpha}\left(-\Delta+k_{1}\right)_{\mu}\left(-\Delta+k_{3}\right)_{\rho} \\
& +\epsilon_{\lambda \mu \beta \rho}\left(-\Delta+k_{2}\right)_{\nu}\left(-\Delta+k_{1}\right)_{\beta}\left(-\Delta+k_{3}\right)_{\rho} \\
& +\epsilon_{\lambda \beta \nu \rho}\left(-\Delta+k_{2}\right)_{\mu}\left(-\Delta+k_{1}\right)_{\beta}\left(-\Delta+k_{3}\right)_{\rho} \\
& +\epsilon_{\alpha \nu \beta \nu}\left(-\Delta+k_{2}\right)_{\alpha}\left(-\Delta+k_{1}\right)_{\beta}\left(-\Delta+k_{3}\right)_{\lambda} \\
& +\epsilon_{\alpha \mu \nu \rho}\left(-\Delta+k_{2}\right)_{\alpha}\left(-\Delta+k_{1}\right)_{\lambda}\left(-\Delta+k_{3}\right)_{\rho} \\
& -\epsilon_{\alpha \beta \nu \rho}\left(-\Delta+k_{2}\right)_{\alpha}\left(-\Delta+k_{1}\right)_{\beta}\left(-\Delta+k_{3}\right)_{\rho} \\
& -\epsilon_{\mu \nu \beta \rho}\left(-\Delta+k_{2}\right)_{\lambda}\left(-\Delta+k_{1}\right)_{\beta}\left(-\Delta+k_{3}\right)_{\rho} \\
& +\frac{\epsilon_{\lambda \alpha \mu \nu}}{2}\left(k_{3}-k_{1}\right)^{2}\left(-\Delta+k_{2}\right)_{\alpha}+\frac{\epsilon_{\lambda \mu \beta \nu}}{2}\left(k_{3}-k_{2}\right)^{2}\left(-\Delta+k_{1}\right)_{\beta} \\
& \left.+\frac{\epsilon_{\lambda \mu \nu \rho}}{2}\left(k_{2}-k_{1}\right)^{2}\left(-\Delta+k_{3}\right)_{\rho}\right\} \frac{-i}{32 \pi^{2}} \frac{1}{M^{2}+m^{2}} \\
& -\frac{i}{16 \pi^{2}} \epsilon_{\lambda \mu \nu \alpha}\left(-k_{3}-k_{2}+2 k_{1}\right)_{\alpha}
\end{aligned}
$$

Considering the relations given in App.A. and App.C., one can easily get the following results

$$
\begin{aligned}
\left(k_{3}-k_{1}\right)_{\mu} T_{\|, \lambda \mu \nu}^{R,(1),\{A V V\}} & =-\frac{1}{4} \frac{i}{4 \pi^{2}} \epsilon_{\lambda \mu \nu \alpha}\left(k_{3}-k_{1}\right)_{\mu}\left(k_{1}-k_{2}\right)_{\alpha} \\
\left(k_{1}-k_{2}\right)_{\nu} T_{\|, \lambda \mu \nu}^{R,(1),\{A V V\}} & =\frac{1}{4} \frac{i}{4 \pi^{2}} \epsilon_{\lambda \mu \nu \alpha}\left(k_{1}-k_{2}\right)_{\nu}\left(k_{3}-k_{1}\right)_{\alpha}
\end{aligned}
$$




$$
\begin{aligned}
\left(k_{3}-k_{2}\right)_{\lambda} T_{\|, \lambda \mu \nu}^{(1),\{A V V\}}= & -\frac{3}{2} \frac{i}{4 \pi^{2}} \epsilon_{\mu \nu \lambda \alpha}\left(k_{3}-k_{1}\right)_{\lambda}\left(k_{1}-k_{2}\right)_{\alpha} \\
& -16 m^{2} \epsilon_{\mu \nu \lambda \alpha}\left(k_{3}-k_{1}\right)_{\lambda}\left(k_{1}-k_{2}\right)_{\alpha} I_{-2,(00)}
\end{aligned}
$$

By considering the perpendicular contribution and the result of the PVV diagram, the anomaly amplitude has the following form

$$
\begin{aligned}
\left(k_{3}-k_{1}\right)_{\mu} T_{\lambda \mu \nu}^{(1),\{A V V\}} & =-\frac{7}{12} \frac{i}{4 \pi^{2}}\left(k_{3}-k_{1}\right)_{\mu}\left(k_{1}-k_{2}\right)_{\alpha} \\
\left(k_{1}-k_{2}\right)_{\nu} T_{\lambda \mu \nu}^{(1),\{A V V\}} & =\frac{7}{12} \frac{i}{4 \pi^{2}}\left(k_{1}-k_{2}\right)_{\nu}\left(k_{3}-k_{1}\right)_{\alpha} \\
\left(k_{3}-k_{2}\right)_{\lambda} T_{\lambda \mu \nu}^{(1),\{A V V\}} & =2 m T_{\mu \nu}^{(1), P V V}-\frac{26}{12} \frac{i}{4 \pi^{2}} \epsilon_{\mu \nu \lambda \alpha}\left(k_{3}-k_{1}\right)_{\lambda}\left(k_{1}-k_{2}\right)_{\alpha}
\end{aligned}
$$

By considering the cross diagram, we obtain the final results

$$
\begin{aligned}
\left(k_{3}-k_{1}\right)_{\mu} T_{\lambda \mu \nu}^{\{A V V\}} & =-\frac{7}{6} \frac{i}{4 \pi^{2}}\left(k_{3}-k_{1}\right)_{\mu}\left(k_{1}-k_{2}\right)_{\alpha} \\
\left(k_{1}-k_{2}\right)_{\nu} T_{\lambda \mu \nu}^{\{A V V\}} & =\frac{7}{6} \frac{i}{4 \pi^{2}}\left(k_{1}-k_{2}\right)_{\nu}\left(k_{3}-k_{1}\right)_{\alpha} \\
\left(k_{3}-k_{2}\right)_{\lambda} T_{\lambda \mu \nu}^{\{A V V\}} & =2 m T_{\mu \nu}^{P V V}-\frac{26}{6} \frac{i}{4 \pi^{2}} \epsilon_{\mu \nu \lambda \alpha}\left(k_{3}-k_{1}\right)_{\lambda}\left(k_{1}-k_{2}\right)_{\alpha}
\end{aligned}
$$

which shows that in the direct calculation of the triangle diagram by adopting the dimensional regularization, there are anomalies in both vector and axial-vector Ward identities even when we treat the gamma trace with the definition of $\gamma_{5}$.

Again to keep the vector currents conserved, one can make a redefinition as done in (6.26). After that, we have the Ward identities for the redefined amplitude

$$
\begin{aligned}
& \left(k_{3}-k_{1}\right)_{\mu} \tilde{T}_{\lambda \mu \nu}^{\{A V V\}}=0 \\
& \left(k_{1}-k_{2}\right)_{\nu} \tilde{T}_{\lambda \mu \nu}^{\{A V V\}}=0 \\
& \left(k_{3}-k_{2}\right)_{\lambda} \tilde{T}_{\lambda \mu \nu}^{\{A V V\}}=2 m T_{\mu \nu}^{P V V}-\frac{i}{2 \pi^{2}} \epsilon_{\mu \nu \lambda \alpha}\left(k_{3}-k_{1}\right)_{\lambda}\left(k_{1}-k_{2}\right)_{\alpha}
\end{aligned}
$$

In conclusion, by adopting the dimensional regularization, the direct calculation of the triangle diagram shows that even one treat the gamma trace with the definition of $\gamma_{5}$ explicitly, both massless QED and massive QED, both vector and axial-vector Ward identities are violated by quantum corrections. But in this case, the magnitudes of the two vector Ward identities are same and different from that of the axial-vector Ward identity. It is then necessary to make a redefinition for the amplitude in order to keep the vector currents conserved, so that the anomaly lives in the axial-vector Ward identity. It is seen that the situation is quite different from the case in the loop regularization and Pauli-Villars scheme. 


\section{CLARIFICATIONS FOR THE LONG-STANDING AMBIGUITIES IN PER- TURBATION CALCULATIONS OF TRIANGLE ANOMALY}

In above sections, we have investigated anomaly by directly calculating the triangle diagram with the most general trace identity (3.16). As shown in section III that there exist in general three independent treatments for carrying out the trace of gamma matrices appearing in the triangle diagram. For considerations of completeness and clarification, we shall investigate other two treatments. In this section, we shall show how the Ward identity anomaly depends on the treatment for the trace of gamma matrices, which then allows us to make a clarification for the long-standing ambiguities in the perturbation calculations of triangle anomaly.

\section{A. Calculation of anomaly with (3.11) in massless QED}

In this subsection, we consider the case that the trace of gamma matrix was evaluated by using (3.10). By using that identity and performing the Dirac algebra, we can express $T_{\lambda \mu \nu}^{(1), A\{V V\}}$ as

$$
\begin{aligned}
T_{\lambda \mu \nu}^{(1), A\{V V\}}= & T_{L, \lambda \mu \nu}^{(1), A\{V\}}+T_{C, \lambda \mu \nu}^{(1), A\{V V\}} \\
T_{L, \lambda \mu \nu}^{(1), A\{V V\}}= & -4 \int \frac{d^{4} k}{(2 \pi)^{4}}\left\{\left\{g_{\mu \nu} \epsilon_{\alpha \beta \lambda \xi}\left(k+k_{1}\right)_{\alpha}\left(k+k_{2}\right)_{\beta}\left(k+k_{3}\right)_{\xi}\right.\right. \\
& -\epsilon_{\nu \beta \lambda \xi}\left(k+k_{1}\right)_{\mu}\left(k+k_{2}\right)_{\beta}\left(k+k_{3}\right)_{\xi}-\epsilon_{\mu \beta \lambda \xi}\left(k+k_{1}\right)_{\nu}\left(k+k_{2}\right)_{\beta}\left(k+k_{3}\right)_{\xi} \\
& \left.-\epsilon_{\mu \alpha \nu \beta}\left(k+k_{1}\right)_{\alpha}\left(k+k_{2}\right)_{\beta}\left(k+k_{3}\right)_{\lambda}-\epsilon_{\mu \alpha \nu \xi}\left(k+k_{1}\right)_{\alpha}\left(k+k_{3}\right)_{\xi}\left(k+k_{2}\right)_{\lambda}\right\} \\
& \times\left[\frac{1}{\left(k+k_{1}\right)^{2}\left(k+k_{2}\right)^{2}\left(k+k_{3}\right)^{2}}\right] \\
& \left.+\frac{\epsilon_{\mu \alpha \nu \lambda}}{2}\left[\frac{\left(k+k_{1}\right)_{\alpha}}{\left(k+k_{1}\right)^{2}\left(k+k_{2}\right)^{2}}+\frac{\left(k+k_{1}\right)_{\alpha}}{\left(k+k_{1}\right)^{2}\left(k+k_{3}\right)^{2}}\right]\right\} \\
T_{C, \lambda \mu \nu}^{(1), A\{V V\}}= & -2 \epsilon_{\mu \nu \alpha \lambda} \int \frac{d^{4} k}{(2 \pi)^{4}} \frac{\left(k_{2}-k_{3}\right)^{2}\left(k+k_{1}\right)_{\alpha}}{\left(k+k_{1}\right)^{2}\left(k+k_{2}\right)^{2}\left(k+k_{3}\right)^{2}}
\end{aligned}
$$

\section{Calculation of anomaly in loop regularization}

After some basic algebra, the regularized amplitude is found to have the following form

$$
\begin{aligned}
T_{\lambda \mu \nu}^{R(1), A\{V V\}} & =T_{0 \lambda \mu \nu}^{R,(1), A\{V V\}}+T_{-2 \lambda \mu \nu}^{R,(1), A\{V V\}} \\
T_{0 \lambda \mu \nu}^{R,(1), A\{V V\}} & =2 \int_{0}^{1} d x_{1} \int_{0}^{x_{1}} d x_{2}\left\{\epsilon_{\mu \lambda \nu \beta}\left(k_{2}+k_{3}-2 k_{1}\right)_{\beta} I_{0}^{R}\left(x_{i}, \mu\right)\right\}
\end{aligned}
$$




$$
\begin{aligned}
& -2 \epsilon_{\mu \alpha \nu \lambda}\left\{\int_{0}^{1} d x\left(-\Delta_{1}+k_{1}\right)_{\alpha} I_{0}^{R}\left(x, \mu_{1}\right)+\int_{0}^{1} d x\left(-\Delta_{3}+k_{1}\right)_{\alpha} I_{0}^{R}\left(x, \mu_{3}\right)\right\} \\
T_{-2 \lambda \mu \nu}^{R,(1), A\{V V\}}= & -4 \int_{0}^{1} d x_{1} \int_{0}^{x_{1}} d x_{2} \\
& \times\left\{-2\left\{\epsilon_{\nu \beta \lambda \xi}\left[\left(-\Delta_{1}+k_{1}\right)_{\mu}\left(-\Delta_{1}+k_{2}\right)_{\beta}\left(-\Delta_{1}+k_{3}\right)_{\xi}\right]\right.\right. \\
& +\epsilon_{\mu \beta \lambda \alpha}\left[\left(-\Delta_{1}+k_{3}\right)_{\alpha}\left(-\Delta_{1}+k_{2}\right)_{\beta}\left(-\Delta_{1}+k_{1}\right)_{\nu}\right] \\
& +\epsilon_{\mu \alpha \nu \beta}\left[\left(-\Delta_{1}+k_{1}\right)_{\alpha}\left(-\Delta_{1}+k_{2}\right)_{\beta}\left(-\Delta_{1}+k_{3}\right)_{\lambda}\right] \\
& \left.+\epsilon_{\mu \alpha \nu \beta}\left[\left(-\Delta_{1}+k_{1}\right)_{\alpha}\left(-\Delta_{1}+k_{3}\right)_{\beta}\left(-\Delta_{1}+k_{2}\right)_{\lambda}\right]\right\} \\
& \left.+\epsilon_{\mu \nu \alpha \lambda}\left\{\left(k_{2}-k_{3}\right)^{2}\left(-\Delta_{1}+k_{1}\right)_{\alpha}\right\}\right\} I_{-2}^{R}\left(x_{i}, \mu\right)
\end{aligned}
$$

We now first check the Ward identity $\left(k_{1}-k_{2}\right)_{\nu} T_{\lambda \mu \nu}^{R, A\{V V\}}$. By using the definitions of $\Delta_{i}$, we have

$$
\begin{aligned}
\left(k_{1}-k_{2}\right)_{\nu} T_{\lambda \mu \nu}^{R,(1), A\{V V\}}= & -2 \epsilon_{\mu \nu \lambda \beta}\left(k_{1}-k_{2}\right)_{\nu}\left(k_{3}-k_{1}\right)_{\beta} \\
& \times\left\{4\left(k_{3}-k_{1}\right) \cdot\left(k_{1}-k_{2}\right)\left[I_{-2,(11)}+I_{-2,(20)}-I_{-2,10}\right]\right. \\
& \left.-4\left(k_{2}-k_{1}\right)^{2}\left[I_{-2,(02)}+I_{-2,(11)}-I_{-2,(01)}\right]+2\left(k_{2}-k_{3}\right)^{2} I_{-2,(10)}\right\} \\
& -2 \epsilon_{\mu \nu \lambda \beta}\left(k_{1}-k_{2}\right)_{\nu}\left(k_{3}-k_{1}\right)_{\beta} I_{0,(00)}
\end{aligned}
$$

By adopting the explicit relations given in App. A, we then obtain

$$
\begin{aligned}
\left(k_{1}-k_{2}\right)_{\nu} T_{\lambda \mu \nu}^{R,(1), A\{V V\}}= & -2 \epsilon_{\mu \nu \lambda \beta}\left(k_{1}-k_{2}\right)_{\nu}\left(k_{3}-k_{1}\right)_{\beta} \\
& \times\left\{\left\{\frac{i}{16 \pi^{2}}\left[e^{-\mu_{s}^{2} / M_{c}^{2}} \int_{0}^{1} d x_{1} \int_{0}^{x_{1}} d x_{2} e^{-M_{1}^{2} / M_{c}^{2}}-\frac{1}{2} Y\left((p+q)^{2}, q^{2}\right)\right]\right.\right. \\
& \left.-q^{2} I_{-2,(10)}-p^{2} I_{-2,(01)}+2 \mu_{s}^{2} I_{-2,(00)}\right\}+2(p+q)^{2} I_{-2,(10)} \\
& +4\left\{p^{2} I_{-2,(01)}-p \cdot q I_{-2,(10)}\right. \\
& +\frac{i}{64 \pi^{2}} e^{-\mu_{s}^{2} / M_{c}^{2}} \int_{0}^{1} d x_{1} \int_{0}^{x_{1}} d x_{2} e^{-M_{1}^{2} / M_{c}^{2}}+\frac{\mu_{s}^{2}}{2} I_{-2,(00)}-\frac{q^{2}}{4} I_{-2,(10)} \\
& \left.\left.-\frac{3 p^{2}}{4} I_{-2,(01)}+\frac{i}{128 \pi^{2}} Y\left((p+q)^{2}, q^{2}\right)-\frac{p^{2}}{2} I_{-2,(10)}\right\}\right\}
\end{aligned}
$$

where the first part in the bracket comes from the difference of two logarithmically divergent integrals and the others come from the convergent integrals. Many terms cancel each other, the final expression gets the following simple form

$$
\begin{aligned}
\left(k_{1}-k_{2}\right)_{\nu} T_{\lambda \mu \nu}^{R,(1), A\{V V\}}= & 8 \mu_{s}^{2} \epsilon_{\mu \lambda \alpha \beta}\left(k_{1}-k_{2}\right)_{\alpha}\left(k_{3}-k_{1}\right)_{\beta} I_{-2,(00)}\left(0, \mu_{s}^{2}\right) \\
& +\frac{i}{4 \pi^{2}} \epsilon_{\mu \lambda \alpha \beta}\left(k_{1}-k_{2}\right)_{\alpha}\left(k_{3}-k_{1}\right)_{\beta} e^{-\mu_{s}^{2} / M_{c}^{2}} \int_{0}^{1} d x_{1} \int_{0}^{x_{1}} d x_{2} e^{-M^{2} / M_{c}^{2}}
\end{aligned}
$$


which is different from the result (3.37) obtained from the general treatment of the gamma trace, where the vector current is found to be conserved automatically.

Similarly, we have the Ward identity for the second vector current

$$
\begin{aligned}
\left(k_{3}-k_{1}\right)_{\mu} T_{\lambda \mu \nu}^{R,(1), A\{V V\}}= & -8 \mu_{s}^{2} \epsilon_{\nu \lambda \alpha \beta}\left(k_{1}-k_{2}\right)_{\alpha}\left(k_{3}-k_{1}\right)_{\beta} I_{-2,(00)}\left(0, \mu_{s}^{2}\right) \\
& -\frac{i}{4 \pi^{2}} \epsilon_{\nu \lambda \alpha \beta}\left(k_{1}-k_{2}\right)_{\alpha}\left(k_{3}-k_{1}\right)_{\beta} e^{-\mu_{s}^{2} / M_{c}^{2}} \int_{0}^{1} d x_{1} \int_{0}^{x_{1}} d x_{2} e^{-M_{1}^{2} / M_{c}^{2}}
\end{aligned}
$$

which has the same result as the first vector current except a total sign that is expected from the treatment in which the two gamma matrices with the Lorentz indices of vector currents are grouped to reduce the number of gamma matrices.

For the divergence of the axial-vector current, we find that

$$
\begin{aligned}
\left(k_{3}-k_{2}\right)_{\lambda} T_{\lambda \mu \nu}^{R,(1), A\{V V\}}= & 4 \epsilon_{\mu \nu \alpha \beta}\left(k_{2}-k_{1}\right)_{\alpha}\left(k_{3}-k_{1}\right)_{\beta} \\
& \times\left\{2\left(k_{2}-k_{1}\right) \cdot\left(k_{3}-k_{2}\right)\left[I_{-2,(20)}-I_{-2,(02)}+I_{-2,(01)}-I_{-2,(10)}\right]\right. \\
& +2\left(k_{3}-k_{2}\right)^{2}\left[I_{-2,(10)}-I_{-2,(20)}+I_{-2,(11)}\right] \\
& \left.-\left(k_{2}-k_{3}\right)^{2}\left[I_{-2,(01)}+I_{-2,(10)}\right]\right\} \\
& -2 \epsilon_{\mu \nu \lambda \beta}\left(k_{1}-k_{2}\right)_{\lambda}\left(k_{3}-k_{1}\right)_{\beta} I_{0,(00)}^{\prime} \\
& -2 \epsilon_{\mu \nu \lambda \beta}\left(k_{1}-k_{2}\right)_{\lambda}\left(k_{3}-k_{1}\right)_{\beta} I_{0,(00)} \\
= & 0
\end{aligned}
$$

which is identically equal to zero, namely there is no anomaly for axial-vector current in this treatment. It is obviously different from the conclusion give by the general treatment (3.39).

As before, we shall consider two interesting cases with $\mu_{s} \neq 0$ and $\mu_{s}=0$.

Firstly, considering the case that $\mu_{s} \neq 0$. Like section III.A, for on-mass shell condition in the massless QED, i.e., $p^{2}=\left(k_{1}-k_{2}\right)^{2}=0, q^{2}=\left(k_{3}-k_{1}\right)^{2}=0,(p+q)^{2}=2 p \cdot q$ is soft with $\mu_{s}^{2} \gg(p+q)^{2}$ and $\mu_{s}^{2} \ll M_{c}^{2} \rightarrow \infty$, the vector current becomes conserved

$$
\begin{aligned}
& \left(k_{3}-k_{1}\right)_{\mu} T_{\lambda \mu \nu}^{R,(1), A\{V V\}}=0 \\
& \left(k_{1}-k_{2}\right)_{\nu} T_{\lambda \mu \nu}^{R,(1), A\{V V\}}=0
\end{aligned}
$$

which shows that in loop regularization with the introduction of nonzero IR cut-off scale $\mu_{s} \neq 0$ for massless QED, the Ward identities of both vector and axial-vector currents become conserved in the conditions that $p^{2}, q^{2},(p+q)^{2} \ll \mu_{s}^{2} \ll M_{c}^{2} \rightarrow \infty$, which comes to 
the same statement made in section III.A. in which the gamma trace was treated with the definition of $\gamma_{5}$.

Secondly, considering the case that $\mu_{s}=0$, we then arrive at the following results

$$
\begin{aligned}
\left(k_{3}-k_{1}\right)_{\mu} T_{\lambda \mu \nu}^{R,(1), A\{V V\}} & =-\frac{i}{4 \pi^{2}} \epsilon_{\nu \lambda \alpha \beta}\left(k_{1}-k_{2}\right)_{\alpha}\left(k_{3}-k_{1}\right)_{\beta} \int_{0}^{1} d x_{1} \int_{0}^{x_{1}} d x_{2} e^{-M_{1}^{2} / M_{c}^{2}}(7.1 \\
\left(k_{1}-k_{2}\right)_{\nu} T_{\lambda \mu \nu}^{R,(1), A\{V V\}} & =\frac{i}{4 \pi^{2}} \epsilon_{\mu \lambda \alpha \beta}\left(k_{1}-k_{2}\right)_{\alpha}\left(k_{3}-k_{1}\right)_{\beta} \int_{0}^{1} d x_{1} \int_{0}^{x_{1}} d x_{2} e^{-M^{2} / M_{c}^{2}} \\
\left(k_{3}-k_{2}\right)_{\lambda} T_{\lambda \mu \nu}^{R,(1), A\{V V\}} & =0
\end{aligned}
$$

Taking $M_{c} \rightarrow \infty$, we yield

$$
\begin{aligned}
\left(k_{3}-k_{1}\right)_{\mu} T_{\lambda \mu \nu}^{R,(1), A\{V V\}} & =-\frac{i}{8 \pi^{2}} \epsilon_{\nu \lambda \alpha \beta}\left(k_{1}-k_{2}\right)_{\alpha}\left(k_{3}-k_{1}\right)_{\beta} \\
\left(k_{1}-k_{2}\right)_{\nu} T_{\lambda \mu \nu}^{R,(1), A\{V V\}} & =\frac{i}{8 \pi^{2}} \epsilon_{\mu \lambda \alpha \beta}\left(k_{1}-k_{2}\right)_{\alpha}\left(k_{3}-k_{1}\right)_{\beta} \\
\left(k_{3}-k_{2}\right)_{\lambda} T_{\lambda \mu \nu}^{R,(1), A\{V V\}} & =0
\end{aligned}
$$

which is again different from the results (3.42 3.44) which were deduced from the general treatment of the gamma trace. It is explicitly seen that in the loop regularization with $\mu_{s}=0$, when the two gamma matrices with the Lorentz indices of the vector current and one gamma between them are grouped to reduce the number of gamma matrices in the trace, the vector currents in massless QED are no longer conserved. To keep the vector Ward identities be conserved, one can simply redefine the physical amplitude via

$$
\widetilde{T}_{\lambda \mu \nu}^{(1), A\{V V\}}\left(k_{3}-k_{1}, k_{1}-k_{2}\right)=T_{\lambda \mu \nu}^{R,(1), A\{V V\}}\left(k_{3}-k_{1}, k_{1}-k_{2}\right)-T_{\lambda \mu \nu}^{(1), A\{V V\}}(0)
$$

with

$$
T_{\lambda \mu \nu}^{(1), A\{V V\}}(0)=-\frac{i}{8 \pi^{2}} \epsilon_{\mu \nu \lambda \alpha}\left[\left(k_{3}-k_{1}\right)_{\alpha}-\left(k_{1}-k_{2}\right)_{\alpha}\right]
$$

Thus the redefined amplitude has the following Ward identities

$$
\begin{aligned}
& \left(k_{3}-k_{1}\right)_{\mu} \widetilde{T}_{\lambda \mu \nu}^{(1), A\{V V\}}=0 \\
& \left(k_{1}-k_{2}\right)_{\nu} \widetilde{T}_{\lambda \mu \nu}^{(1), A\{V V\}}=0 \\
& \left(k_{3}-k_{2}\right)_{\lambda} \widetilde{T}_{\lambda \mu \nu}^{(1), A\{V V\}}=-\frac{i}{4 \pi^{2}} \epsilon_{\mu \nu \alpha \beta}\left(k_{3}-k_{1}\right)_{\alpha}\left(k_{1}-k_{2}\right)_{\beta}
\end{aligned}
$$

By including the cross diagrams, we finally obtain the Ward identities with anomaly of axial-vector current

$$
\left(k_{3}-k_{1}\right)_{\mu} \widetilde{T}_{\lambda \mu \nu}^{A\{V V\}}=0
$$




$$
\begin{aligned}
& \left(k_{1}-k_{2}\right)_{\nu} \widetilde{T}_{\lambda \mu \nu}^{A\{V V\}}=0 \\
& \left(k_{3}-k_{2}\right)_{\lambda} \widetilde{T}_{\lambda \mu \nu}^{A\{V V\}}=-\frac{i}{2 \pi^{2}} \epsilon_{\mu \nu \alpha \beta}\left(k_{3}-k_{1}\right)_{\alpha}\left(k_{1}-k_{2}\right)_{\beta}
\end{aligned}
$$

Its operator form can be expressed as

$$
\begin{aligned}
\partial_{\mu} V_{\mu}(x) & =0 \\
\partial_{\mu} A_{\mu}(x) & =\frac{e^{2}}{8 \pi^{2}} F^{\mu \nu}(x) \widetilde{F}_{\mu \nu}(x)
\end{aligned}
$$

In conclusion, it is seen from the above analysis that the IR-cut off $\mu_{s}$ still plays an important role for an anomaly-free treatment in the present case. While the Ward identity anomaly of currents with treating the trace of gamma matrices with (3.11) is different from the case with the general relation (3.16). When we treat using the relation (3.11), the two vector Ward identities are violated by quantum corrections, the axial-vector Ward identity becomes conserved. To keep the vector current conserved, one needs to make a redefinition for the amplitude, so that the anomaly appears in the axial-vector Ward identity.

\section{Calculation of anomaly by using Pauli-Villars regularization}

When applying for the Pauli-Villars regularization, the regularized amplitude reads

$$
\begin{aligned}
T_{L, \lambda \mu \nu}^{R,(1), A\{V V\}}= & -4 \sum_{i=0}^{2} C_{i} \int \frac{d^{4} k}{(2 \pi)^{4}}\left\{\left\{g_{\mu \nu} \epsilon_{\alpha \beta \lambda \xi}\left(k+k_{1}\right)_{\alpha}\left(k+k_{2}\right)_{\beta}\left(k+k_{3}\right)_{\xi}\right.\right. \\
& -\epsilon_{\nu \beta \lambda \xi}\left(k+k_{1}\right)_{\mu}\left(k+k_{2}\right)_{\beta}\left(k+k_{3}\right)_{\xi}-\epsilon_{\mu \beta \lambda \xi}\left(k+k_{1}\right)_{\nu}\left(k+k_{2}\right)_{\beta}\left(k+k_{3}\right)_{\xi} \\
& \left.-\epsilon_{\mu \alpha \nu \beta}\left(k+k_{1}\right)_{\alpha}\left(k+k_{3}\right)_{\beta}\left(k+k_{2}\right)_{\lambda}-\epsilon_{\mu \alpha \nu \xi}\left(k+k_{1}\right)_{\alpha}\left(k+k_{2}\right)_{\xi}\left(k+k_{3}\right)_{\lambda}\right\} \\
& \times\left[\frac{1}{\left[\left(k+k_{1}\right)^{2}-m_{i}^{2}\right]\left[\left(k+k_{2}\right)^{2}-m_{i}^{2}\right]\left[\left(k+k_{3}\right)^{2}-m_{i}^{2}\right]}\right] \\
& +\frac{\epsilon_{\mu \alpha \nu \lambda}}{2}\left[\frac{\left(k+k_{1}\right)_{\alpha}}{\left[\left(k+k_{1}\right)^{2}-m_{i}^{2}\right]\left[\left(k+k_{2}\right)^{2}-m_{i}^{2}\right]}\right. \\
& \left.\left.+\frac{\left(k+k_{1}\right)_{\alpha}}{\left[\left(k+k_{1}\right)^{2}-m_{i}^{2}\right]\left[\left(k+k_{3}\right)^{2}-m_{i}^{2}\right]}\right]\right\} \\
T_{C, \lambda \mu \nu}^{(1), A\{V V\}}= & -2 \epsilon_{\mu \nu \alpha \lambda} \sum_{i=0}^{2} C_{i} \int \frac{d^{4} k}{(2 \pi)^{4}} \\
& \times \frac{2 m_{i}^{2}\left(k+k_{2}\right)_{\alpha}-\left[4 m_{i}^{2}-\left(k_{2}-k_{3}\right)^{2}\right]\left(k+k_{1}\right)_{\alpha}+2 m_{i}^{2}\left(k+k_{3}\right)_{\alpha}}{\left[\left(k+k_{1}\right)^{2}-m_{i}^{2}\right]\left[\left(k+k_{2}\right)^{2}-m_{i}^{2}\right]\left[\left(k+k_{3}\right)^{2}-m_{i}^{2}\right]}
\end{aligned}
$$

with the initial condition $c_{0}=1$ and $m_{0}=0$. After adopting Feynman parametrization and shifting the integration variables, we obtain the similar expressions as those in loop 
regularization

$$
\begin{aligned}
T_{\lambda \mu \nu}^{(1), A\{V V\}}= & T_{0 \lambda \mu \nu}^{R,(1), A\{V V\}}+T_{-2 \lambda \mu \nu}^{R,(1), A\{V V\}} \\
T_{0 \lambda \mu \nu}^{R,(1), A\{V V\}}= & -2 \sum_{i=0}^{2} C_{i} \int_{0}^{1} d x_{1} \int_{0}^{x_{1}} d x_{2}\left\{\epsilon_{\mu \lambda \nu \beta}\left(k_{2}+k_{3}-2 k_{1}\right)_{\beta} \int \frac{d^{4} k}{(2 \pi)^{2}} \frac{k^{2}}{\left[k^{2}-M_{i}^{2}\right]^{3}}\right\} \\
& -2 \epsilon_{\mu \alpha \nu \lambda}\left\{\sum_{i=0}^{2} C_{i} \int_{0}^{1} d x\left(-\Delta_{2}+k_{1}\right)_{\alpha} \int \frac{d^{4} k}{(2 \pi)^{2}} \frac{1}{\left[k^{2}-M_{1 i}^{2}\right]^{2}}\right. \\
& \left.+\sum_{i=0}^{2} C_{i} \int_{0}^{1} d x\left(-\Delta_{3}+k_{1}\right)_{\alpha} \int \frac{d^{4} k}{(2 \pi)^{2}} \frac{1}{\left[k^{2}-M_{3 i}^{2}\right]^{2}}\right\} \\
T_{-2 \lambda \mu \nu}^{R,(1), A\{V\}=} & 4 \sum_{i=0}^{2} C_{i} \int_{0}^{1} d x_{1} \int_{0}^{x_{1}} d x_{2} \\
& \times\left\{2 \left\{\epsilon_{\mu \beta \lambda \xi}\left[\left(-\Delta_{1}+k_{1}\right)_{\nu}\left(-\Delta_{1}+k_{2}\right)_{\beta}\left(-\Delta_{1}+k_{3}\right)_{\xi}\right]\right.\right. \\
& +\epsilon_{\nu \beta \lambda \alpha}\left[\left(-\Delta_{1}+k_{3}\right)_{\alpha}\left(-\Delta_{1}+k_{2}\right)_{\beta}\left(-\Delta_{1}+k_{1}\right)_{\mu}\right] \\
& +\epsilon_{\mu \alpha \nu \beta}\left[\left(-\Delta_{1}+k_{1}\right)_{\alpha}\left(-\Delta_{1}+k_{2}\right)_{\beta}\left(-\Delta_{1}+k_{3}\right)_{\lambda}\right] \\
& \left.+\epsilon_{\mu \alpha \nu \beta}\left[\left(-\Delta_{1}+k_{1}\right)_{\alpha}\left(-\Delta_{1}+k_{3}\right)_{\beta}\left(-\Delta_{1}+k_{2}\right)_{\lambda}\right]\right\} \\
& -2 \epsilon_{\mu \nu \alpha \lambda} m_{i}^{2}\left(k_{2}+k_{3}-2 k_{1}\right)_{\alpha} \\
& \left.-\epsilon_{\mu \nu \alpha \lambda}\left(k_{2}-k_{3}\right)^{2}\left(-\Delta_{1}+k_{1}\right)_{\alpha}\right\} \int \frac{d^{4} k}{(2 \pi)^{2}} \frac{1}{\left[k^{2}-M_{i}^{2}\right]^{3}}
\end{aligned}
$$

By taking the useful relations given in App. B and also in App. A with $\mu_{s}=0, m=0$ and $M_{c} \rightarrow \infty$, we have

$$
\begin{aligned}
\left(k_{3}-k_{1}\right)_{\mu} T_{\lambda \mu \nu}^{R,(1), A\{V V\}} & =\sum_{i=0}^{2} C_{i}\left(\frac{-i}{8 \pi^{2}}\right) \epsilon_{\nu \lambda \alpha \beta}\left(k_{1}-k_{2}\right)_{\alpha}\left(k_{3}-k_{1}\right)_{\beta}=0 \\
\left(k_{1}-k_{2}\right)_{\nu} T_{\lambda \mu \nu}^{R,(1), A\{V V\}} & =\sum_{i=0}^{2} C_{i}\left(\frac{i}{8 \pi^{2}}\right) \epsilon_{\mu \lambda \alpha \beta}\left(k_{1}-k_{2}\right)_{\alpha}\left(k_{3}-k_{1}\right)_{\beta}=0 \\
\left(k_{3}-k_{2}\right)_{\lambda} T_{\lambda \mu \nu}^{R,(1), A\{V V\}} & =\sum_{i=0}^{2} C_{i}\left[2 m_{i} T_{\mu \nu}^{(1), P V V}\left(m_{i}\right)\right]
\end{aligned}
$$

which shows that in Pauli-Villars regularization the vector Ward identities are preserved automatically. Although this is the same as eqs.(5.7/5.9) obtained by using the general trace relation (3.16), while the mechanism becomes different. In the present case, there are anomalies in both the original vector currents and the regulator vector currents of superheavy fields, but the sum of their anomalous terms cancel each other. In the previous case, the vector currents of both the original fermion and regulator fermions are conserved separately. 
For axial-vector Ward identity, by using the same condition as (5.11), we have

$$
\sum_{i=0}^{2} C_{i}\left[2 m_{i} T_{\mu \nu}^{(1), P V V}\left(m_{i}\right)\right]=\frac{i}{4 \pi^{2}} \epsilon_{\mu \nu \alpha \beta}\left(k_{1}-k_{2}\right)_{\alpha}\left(k_{3}-k_{1}\right)_{\beta}
$$

It is seen again that the source of anomaly in Pauli-Villars regularization arises from the heavy regulator fermion loops.

\section{Calculation of anomaly by using dimensional regularization}

By using (3.10) and considering the amplitude (6.6), one can write the parallel part of the amplitude (6.7) as

$$
\begin{aligned}
T_{\|, L, \lambda \mu \nu}^{R,(1), A\{V V\}}= & -4 \int \frac{d^{n} k}{(2 \pi)^{n}}\left\{\left\{g_{\mu \nu} \epsilon_{\alpha \beta \lambda \xi}\left(k_{\|}+k_{1}\right)_{\alpha}\left(k_{\|}+k_{2}\right)_{\beta}\left(k_{\|}+k_{3}\right)_{\xi}\right.\right. \\
& -\epsilon_{\nu \beta \lambda \xi}\left(k_{\|}+k_{1}\right)_{\mu}\left(k_{\|}+k_{2}\right)_{\beta}\left(k_{\|}+k_{3}\right)_{\xi}-\epsilon_{\mu \beta \lambda \xi}\left(k_{\|}+k_{1}\right)_{\nu}\left(k_{\|}+k_{2}\right)_{\beta}\left(k_{\|}+k_{3}\right)_{\xi} \\
& \left.-\epsilon_{\mu \alpha \nu \beta}\left(k_{\|}+k_{1}\right)_{\alpha}\left(k_{\|}+k_{2}\right)_{\beta}\left(k_{\|}+k_{3}\right)_{\lambda}-\epsilon_{\mu \alpha \nu \xi}\left(k_{\|}+k_{1}\right)_{\alpha}\left(k_{\|}+k_{3}\right)_{\xi}\left(k_{\|}+k_{2}\right)_{\lambda}\right\} \\
& \times\left[\frac{1}{\left(k+k_{1}\right)^{2}\left(k+k_{2}\right)^{2}\left(k+k_{3}\right)^{2}}\right] \\
& \left.+\frac{\epsilon_{\mu \alpha \nu \lambda}}{2}\left[\frac{\left(k_{\|}+k_{1}\right)_{\alpha}}{\left(k+k_{1}\right)^{2}\left(k+k_{2}\right)^{2}}+\frac{\left(k_{\|}+k_{1}\right)_{\alpha}}{\left(k+k_{1}\right)^{2}\left(k+k_{3}\right)^{2}}\right]\right\} \\
T_{\|, C, \lambda \mu \nu}^{(1), A\{V V\}}= & -2 \epsilon_{\mu \nu \alpha \lambda} \int \frac{d^{n} k}{(2 \pi)^{n}} \frac{\left(k_{2}-k_{3}\right)^{2}\left(k_{\|}+k_{1}\right)_{\alpha}}{\left(k+k_{1}\right)^{2}\left(k+k_{2}\right)^{2}\left(k+k_{3}\right)^{2}}
\end{aligned}
$$

By adopting Feynman parametrization, shifting the integration variables and using App. C., we obtain

$$
\begin{aligned}
T_{\|, \lambda \mu \nu}^{(1), A\{V V\}}= & T_{\|, 0, \lambda \mu \nu}^{(1), A\{V V\}}+T_{\|,-2, \lambda \mu \nu}^{(1), A\{V V\}} \\
T_{\|, 0, \lambda \mu \nu}^{(1), A\{V V\}}= & 2 \int_{0}^{1} d x_{1} \int_{0}^{x_{1}} d x_{2} \epsilon_{\mu \lambda \nu \beta}\left(k_{2}+k_{3}-2 k_{1}\right)_{\beta} \frac{i}{16 \pi^{2}}\left[-\ln M^{2}\right] \\
& -2 \epsilon_{\mu \lambda \nu \alpha}\left\{\int_{0}^{1} d x x\left(k_{2}-k_{1}\right)_{\alpha} \frac{i}{16 \pi^{2}}\left[-\ln M_{1}^{2}\right]\right. \\
& \left.+\int_{0}^{1} d x x\left(k_{3}-k_{1}\right)_{\alpha} \frac{i}{16 \pi^{2}}\left[-\ln M_{3}^{2}\right]\right\} \\
T_{\|,-2, \lambda \mu \nu}^{R,(1), A\{V V\}}= & -4 \int_{0}^{1} d x_{1} \int_{0}^{x_{1}} d x_{2} \\
& \times\left\{-2\left\{\epsilon_{\nu \beta \lambda \xi}\left[\left(-\Delta_{1}+k_{1}\right)_{\mu}\left(-\Delta_{1}+k_{2}\right)_{\beta}\left(-\Delta_{1}+k_{3}\right)_{\xi}\right]\right.\right. \\
& +\epsilon_{\mu \beta \lambda \alpha}\left[\left(-\Delta_{1}+k_{3}\right)_{\alpha}\left(-\Delta_{1}+k_{2}\right)_{\beta}\left(-\Delta_{1}+k_{1}\right)_{\nu}\right] \\
& +\epsilon_{\mu \alpha \nu \beta}\left[\left(-\Delta_{1}+k_{1}\right)_{\alpha}\left(-\Delta_{1}+k_{2}\right)_{\beta}\left(-\Delta_{1}+k_{3}\right)_{\lambda}\right] \\
& \left.+\epsilon_{\mu \alpha \nu \beta}\left[\left(-\Delta_{1}+k_{1}\right)_{\alpha}\left(-\Delta_{1}+k_{3}\right)_{\beta}\left(-\Delta_{1}+k_{2}\right)_{\lambda}\right]\right\}
\end{aligned}
$$




$$
\begin{aligned}
& \left.+\epsilon_{\mu \nu \alpha \lambda}\left\{\left(k_{2}-k_{3}\right)^{2}\left(-\Delta_{1}+k_{1}\right)_{\alpha}\right\}\right\} \frac{-i}{32 \pi^{2}} \frac{1}{M^{2}} \\
& +\frac{i}{32 \pi^{2}} \epsilon_{\mu \lambda \nu \beta}\left(k_{2}+k_{3}-2 k_{1}\right)_{\beta}
\end{aligned}
$$

where $T_{\|, 0, \lambda \mu \nu}^{(1), A V V}$ and $T_{\|,-2, \lambda \mu \nu}^{R,(1), A V V}$ are corresponding to the logarithemic and convergent terms in (7.32) and (7.33). $x$ and $x_{i}$ are Feynman parameters. By using the relations given in App. A. with $\mu_{s}^{2}=0$ and $M_{c}^{2} \rightarrow \infty$, we find that the contributions from the $k_{\|}$have the following forms

$$
\begin{aligned}
\left(k_{3}-k_{1}\right)_{\mu} T_{\|, \lambda \mu \nu}^{R,(1), A\{V V\}} & =\frac{10}{16} \frac{-i}{4 \pi^{2}} \epsilon_{\nu \lambda \alpha \beta}\left(k_{1}-k_{2}\right)_{\alpha}\left(k_{3}-k_{1}\right)_{\beta} \\
\left(k_{1}-k_{2}\right)_{\nu} T_{\|, \lambda \mu \nu}^{R,(1), A\{V V\}} & =\frac{10}{16} \frac{i}{4 \pi^{2}} \epsilon_{\mu \lambda \alpha \beta}\left(k_{1}-k_{2}\right)_{\alpha}\left(k_{3}-k_{1}\right)_{\beta} \\
\left(k_{3}-k_{2}\right)_{\lambda} T_{\|, \lambda \mu \nu}^{R,(1), A\{V V\}} & =\frac{4}{16} \frac{i}{4 \pi^{2}} \epsilon_{\mu \lambda \nu \beta}\left(k_{1}-k_{2}\right)_{\lambda}\left(k_{3}-k_{1}\right)_{\beta}
\end{aligned}
$$

By including the perpendicular part, we yield the following results

$$
\begin{aligned}
\left(k_{3}-k_{1}\right)_{\mu} T_{\lambda \mu \nu}^{R,(1), A\{V V\}} & =\frac{14}{48} \frac{-i}{4 \pi^{2}} \epsilon_{\nu \lambda \alpha \beta}\left(k_{1}-k_{2}\right)_{\alpha}\left(k_{3}-k_{1}\right)_{\beta} \\
\left(k_{1}-k_{2}\right)_{\nu} T_{\lambda \mu \nu}^{R,(1), A\{V V\}} & =\frac{14}{48} \frac{i}{4 \pi^{2}} \epsilon_{\mu \lambda \alpha \beta}\left(k_{1}-k_{2}\right)_{\alpha}\left(k_{3}-k_{1}\right)_{\beta} \\
\left(k_{3}-k_{2}\right)_{\lambda} T_{\lambda \mu \nu}^{R,(1), A\{V V\}} & =\frac{20}{48} \frac{i}{4 \pi^{2}} \epsilon_{\mu \nu \lambda \beta}\left(k_{1}-k_{2}\right)_{\lambda}\left(k_{3}-k_{1}\right)_{\beta}
\end{aligned}
$$

which shows that it is similar to the previous case indicated in (6.22,6.24), namely all three currents have anomalies, but their magnitudes are different as seen from the numerical coefficients.

Including the cross diagrams, we arrive at the final results

$$
\begin{aligned}
\left(k_{3}-k_{1}\right)_{\mu} T_{\lambda \mu \nu}^{R, A\{V V\}} & =\frac{14}{24} \frac{-i}{4 \pi^{2}} \epsilon_{\nu \lambda \alpha \beta}\left(k_{1}-k_{2}\right)_{\alpha}\left(k_{3}-k_{1}\right)_{\beta} \\
\left(k_{1}-k_{2}\right)_{\nu} T_{\lambda \mu \nu}^{R, A\{V V\}} & =\frac{14}{24} \frac{i}{4 \pi^{2}} \epsilon_{\mu \lambda \alpha \beta}\left(k_{1}-k_{2}\right)_{\alpha}\left(k_{3}-k_{1}\right)_{\beta} \\
\left(k_{3}-k_{2}\right)_{\lambda} T_{\lambda \mu \nu}^{R, A\{V V\}} & =\frac{20}{24} \frac{i}{4 \pi^{2}} \epsilon_{\mu \nu \lambda \beta}\left(k_{1}-k_{2}\right)_{\lambda}\left(k_{3}-k_{1}\right)_{\beta}
\end{aligned}
$$

Again both the vector Ward identities and axial-vector identity are violated. To keep the vector current be conserved, the following redefinition for the amplitudes is needed

$$
\widetilde{T}_{\lambda \mu \nu}^{(1), A\{V V\}}\left(k_{3}-k_{1}, k_{1}-k_{2}\right)=T_{\lambda \mu \nu}^{(1), A\{V V\}}\left(k_{3}-k_{1}, k_{1}-k_{2}\right)-T_{\lambda \mu \nu}^{(1), A\{V V\}}(0)
$$

with

$$
T_{\lambda \mu \nu}^{(1), A\{V V\}}(0)=\frac{14}{24} \frac{-i}{4 \pi^{2}} \epsilon_{\mu \nu \lambda \alpha}\left[\left(k_{3}-k_{1}\right)_{\alpha}-\left(k_{1}-k_{2}\right)_{\alpha}\right]
$$


One can check that the redefined amplitude has the Ward identity anomaly only in the axial-vector current

$$
\begin{aligned}
& \left(k_{3}-k_{1}\right)_{\mu} \widetilde{T}_{\lambda \mu \nu}^{A\{V V\}}=0 \\
& \left(k_{1}-k_{2}\right)_{\nu} \widetilde{T}_{\lambda \mu \nu}^{A\{V V\}}=0 \\
& \left(k_{3}-k_{2}\right)_{\lambda} \widetilde{T}_{\lambda \mu \nu}^{A\{V V\}}=-\frac{i}{2 \pi^{2}} \epsilon_{\mu \nu \alpha \beta}\left(k_{3}-k_{1}\right)_{\alpha}\left(k_{1}-k_{2}\right)_{\beta}
\end{aligned}
$$

From the above explicit calculations, it is seen that in the dimensional regularization the divergences of both the vector and axial-vector currents are violated by quantum corrections. The quantum corrections also depend on both the original four dimensions and the extended dimensions. This is similar to the case where the trace was evaluated by using the definition of $\gamma_{5}$ explicitly. However, the magnitudes of anomalous terms are different in these two treatments, so that the redefinitions of the amplitudes are also different, although the final form is the same when normalizing to the conserved vector currents.

\section{B. Calculation of anomaly by using (3.13) in massless QED}

Instead of treating the trace of gamma matrix with the relation (3.13), we now consider in this subsection the case that the trace of gamma matrix is evaluated with the relation (3.13).

After Dirac algebra, we can write $T_{\lambda \mu \nu}^{(1), A V V}$ as

$$
\begin{aligned}
T_{\lambda \mu \nu}^{(1),\{A V\} V}= & T_{L, \lambda \mu \nu}^{(1),\{A V\} V}+T_{C, \lambda \mu \nu}^{(1),\{A V\} V} \\
T_{L, \lambda \mu \nu}^{(1),\{A V\} V}= & -4 \int \frac{d^{4} k}{(2 \pi)^{4}}\left\{\left\{g_{\nu \lambda} \epsilon_{\alpha \beta \mu \xi}\left(k+k_{1}\right)_{\alpha}\left(k+k_{2}\right)_{\beta}\left(k+k_{3}\right)_{\xi}\right.\right. \\
& +\epsilon_{\nu \beta \mu \xi}\left(k+k_{1}\right)_{\beta}\left(k+k_{2}\right)_{\lambda}\left(k+k_{3}\right)_{\xi}+\epsilon_{\lambda \beta \mu \xi}\left(k+k_{1}\right)_{\beta}\left(k+k_{2}\right)_{\nu}\left(k+k_{3}\right)_{\xi} \\
& \left.+\epsilon_{\lambda \alpha \nu \beta}\left(k+k_{1}\right)_{\beta}\left(k+k_{2}\right)_{\alpha}\left(k+k_{3}\right)_{\mu}+\epsilon_{\lambda \alpha \nu \xi}\left(k+k_{1}\right)_{\mu}\left(k+k_{2}\right)_{\alpha}\left(k+k_{3}\right)_{\xi}\right\} \\
& \times\left[\frac{1}{\left(k+k_{1}\right)^{2}\left(k+k_{2}\right)^{2}\left(k+k_{3}\right)^{2}}\right] \\
& \left.-\frac{\epsilon_{\lambda \alpha \nu \mu}}{2}\left[\frac{\left(k+k_{2}\right)_{\alpha}}{\left(k+k_{2}\right)^{2}\left(k+k_{3}\right)^{2}}+\frac{\left(k+k_{2}\right)_{\alpha}}{\left(k+k_{2}\right)^{2}\left(k+k_{1}\right)^{2}}\right]\right\} \\
T_{C, \lambda \mu \nu}^{(1),\{A V\} V}= & -2 \epsilon_{\lambda \alpha \nu \mu} \int \frac{d^{4} k}{(2 \pi)^{4}} \frac{\left(k_{3}-k_{1}\right)^{2}\left(k+k_{2}\right)_{\alpha}}{\left(k+k_{1}\right)^{2}\left(k+k_{2}\right)^{2}\left(k+k_{3}\right)^{2}}
\end{aligned}
$$

Again we will check the Ward identities by using three regularization schemes. 


\section{Calculation of anomaly in the loop regularization}

Applying for the loop regularization, we have after some basic algebra that

$$
\begin{aligned}
T_{\lambda \mu \nu}^{R,(1),\{A V\} V}= & T_{0 \lambda \mu \nu}^{(1),\{A V\} V}+T_{-2 \lambda \mu \nu}^{(1),\{A V\} V} \\
T_{0 \lambda \mu \nu}^{R,(1),\{A V\} V}= & 2 \int_{0}^{1} d x_{1} \int_{0}^{x_{1}} d x_{2}\left\{\epsilon_{\mu \lambda \nu \beta}\left(k_{3}+k_{1}-2 k_{2}\right)_{\beta} I_{0}^{R}\left(x_{i}, \mu\right)\right\} \\
& -2 \epsilon_{\mu \alpha \nu \lambda}\left\{\int_{0}^{1} d x\left(-\Delta_{2}+k_{2}\right)_{\alpha} I_{0}^{R}\left(x, \mu_{2}\right)+\int_{0}^{1} d x\left(-\Delta_{1}+k_{2}\right)_{\alpha} I_{0}^{R}\left(x, \mu_{1}\right)\right\} \\
T_{-2 \lambda \mu \nu}^{R,(1),\{A V\} V}= & -4 \int_{0}^{1} d x_{1} \int_{0}^{x_{1}} d x_{2} \times\left\{-2\left\{\epsilon_{\mu \beta \nu \xi}\left[\left(-\Delta+k_{1}\right)_{\beta}\left(-\Delta+k_{2}\right)_{\lambda}\left(-\Delta+k_{3}\right)_{\xi}\right]\right.\right. \\
& +\epsilon_{\mu \beta \lambda \xi}\left[\left(-\Delta+k_{1}\right)_{\beta}\left(-\Delta+k_{2}\right)_{\nu}\left(-\Delta+k_{3}\right)_{\xi}\right] \\
& +\epsilon_{\nu \alpha \lambda \beta}\left[\left(-\Delta+k_{1}\right)_{\beta}\left(-\Delta+k_{2}\right)_{\alpha}\left(-\Delta+k_{3}\right)_{\mu}\right] \\
& \left.+\epsilon_{\nu \alpha \lambda \xi}\left[\left(-\Delta+k_{1}\right)_{\mu}\left(-\Delta+k_{3}\right)_{\xi}\left(-\Delta+k_{2}\right)_{\alpha}\right]\right\} \\
& \left.+\epsilon_{\lambda \alpha \nu \mu}\left\{\left(k_{3}-k_{1}\right)^{2}\left(-\Delta+k_{2}\right)_{\alpha}\right\}\right\} I_{-2}^{R}\left(x_{i}, \mu\right)
\end{aligned}
$$

From the definitions of $\Delta$ and $\Delta_{i}$, we have

$$
\begin{aligned}
\left(k_{1}-k_{2}\right)_{\nu} T_{\lambda \mu \nu}^{R,(1),\{A V\} V}= & -2 \epsilon_{\nu \lambda \mu \xi}\left(k_{1}-k_{2}\right)_{\nu}\left(k_{3}-k_{1}\right)_{\xi} \\
& \times\left\{4 \left[\left(k_{1}-k_{2}\right)^{2} I_{-2,(01)}-\left(k_{1}-k_{2}\right)^{2} I_{-2,(02)}\right.\right. \\
& \left.\left.+\left(k_{3}-k_{1}\right) \cdot\left(k_{1}-k_{2}\right) I_{-2,(11)}\right]+2\left(k_{3}-k_{1}\right)^{2} I_{-2,(10)}\right\} \\
& -2 \epsilon_{\nu \lambda \mu \xi}\left(k_{1}-k_{2}\right)_{\nu}\left(k_{3}-k_{1}\right)_{\xi}\left\{I_{0,(00)}+\frac{i}{32 \pi^{2}} Y\left((p+q)^{2}, q^{2}\right)\right\} \\
\left(k_{3}-k_{2}\right)_{\lambda} T_{\lambda \mu \nu}^{R,(1),\{A V\} V}= & -2 \epsilon_{\nu \lambda \mu \xi}\left(k_{1}-k_{2}\right)_{\nu}\left(k_{3}-k_{1}\right)_{\xi} \\
& \times\left\{4 \left\{\left(k_{1}-k_{2}\right)^{2}\left[I_{-2,01}-I_{-2,(02)}\right]\right.\right. \\
& +\left(k_{1}-k_{2}\right) \cdot\left(k_{3}-k_{1}\right)\left[I_{-2,(01)}-I_{-2,(02)}+I_{-2,(11)}\right] \\
& \left.+\left(k_{3}-k_{1}\right)^{2} I_{-2,(11)}\right\} \\
& +2\left\{\left(k_{3}-k_{1}\right)^{2}\left[I_{-2,(00)}-I_{-2,(01)}-I_{-2,(10)}\right\}\right\} \\
& -2 \epsilon_{\nu \lambda \mu \xi}\left(k_{1}-k_{2}\right)_{\nu}\left(k_{3}-k_{1}\right)_{\xi} I_{0,(00)}^{\prime}
\end{aligned}
$$

where the factor $\left(i / 32 \pi^{2}\right) Y\left((p+q)^{2}, q^{2}\right)$ in the last term of (17.46) $)$ comes from:

$$
\int_{0}^{1} d x x\left[I_{0}^{R}\left(x, \mu_{3}\right)-I_{0}^{R}\left(x, \mu_{2}\right)\right]=\frac{i}{16 \pi^{2}} \frac{1}{2} Y\left((p+q)^{2}, q^{2}\right)
$$

With the help of the relations given in App.A. at $m^{2}=0$, the above results are simplified to be

$$
\left(k_{1}-k_{2}\right)_{\nu} T_{\lambda \mu \nu}^{R,(1),\{A V\} V}=-8 \mu_{s}^{2} \epsilon_{\nu \lambda \mu \xi}\left(k_{1}-k_{2}\right)_{\nu}\left(k_{3}-k_{1}\right)_{\xi} I_{-2,(00)}
$$




$$
\begin{aligned}
& -\frac{i}{4 \pi^{2}} \epsilon_{\nu \lambda \mu \xi}\left(k_{1}-k_{2}\right)_{\nu}\left(k_{3}-k_{1}\right)_{\xi} e^{-\mu_{s}^{2} / M_{c}^{2}} \int_{0}^{1} d x_{1} \int_{0}^{x_{1}} d x_{2} e^{-M^{2} / M_{c}^{2}} \\
\left(k_{3}-k_{2}\right)_{\lambda} T_{\lambda \mu \nu}^{R,(1),\{A V\} V}= & 8 \mu_{s}^{2} \epsilon_{\nu \lambda \mu \xi}\left(k_{1}-k_{2}\right)_{\lambda}\left(k_{3}-k_{1}\right)_{\xi} I_{-2,(00)} \\
& +\frac{i}{4 \pi^{2}} \epsilon_{\nu \lambda \mu \xi}\left(k_{1}-k_{2}\right)_{\lambda}\left(k_{3}-k_{1}\right)_{\xi} e^{-\mu_{s}^{2} / M_{c}^{2}} \int_{0}^{1} d x_{1} \int_{0}^{x_{1}} d x_{2} e^{-M^{2} / M_{c}^{2}}
\end{aligned}
$$

It is seen that the first identity is the same as (7.6) except a total sign, but different from (3.37). The second identity is different from either eq.(17.8) or eq.(3.39).

By making the similar evaluation, it is easy to check the Ward identity of the remaining vector current. We find that

$$
\left(k_{3}-k_{1}\right)_{\mu} T_{\lambda \mu \nu}^{R,(1),\{A V\} V}=0
$$

which shows that the Ward identity of this vector current is preserved automatically, which is the same as eq. (3.38) but different from eq. (7.7).

Following the previous subsection, we first consider the case with $\mu_{s} \neq 0$ in massless QED. As in section III.A. with the conditions $p^{2}=\left(k_{1}-k_{2}\right)^{2}=0, q^{2}=\left(k_{3}-k_{1}\right)^{2}=0$ and $(p+q)^{2}=2 p \cdot q$ is soft with $\mu_{s}^{2} \gg\left(p+q^{2}\right)$ and $\mu_{s}^{2} \ll M_{c}^{2} \rightarrow \infty$, the vector current and axial-vector current become conserved

$$
\begin{aligned}
& \left(k_{1}-k_{2}\right)_{\nu} T_{\lambda \mu \nu}^{R,(1),\{A V\} V}=0 \\
& \left(k_{3}-k_{2}\right)_{\lambda} T_{\lambda \mu \nu}^{R,(1),\{A V\} V}=0
\end{aligned}
$$

which means that in the calculation with the trace relation (3.13), all the three Ward identities, under the condition that $p^{2}, q^{2},(p+q)^{2} \ll \mu_{s}^{2} \ll M_{c}^{2}$, are conserved by the quantum corrections.

It is noticed that the conclusions under such a condition are the same for all the three treatments. Nevertheless, one may see that there exist subtle differences among the three treatments: in the treatment with trace relation (3.11), the axial-vector is conserved automatically; in the treatment with relation (3.13), one of the vector current becomes conserved automatically; in the treatment with relation (3.16), two vector currents are conserved automatically.

We now consider the second case $\mu_{s}=0$. In this case, three Ward identities of the vector and axial-vector currents are found to have the following forms

$$
\left(k_{3}-k_{1}\right)_{\mu} T_{\lambda \mu \nu}^{R,(1),\{A V\} V}=0
$$




$$
\begin{aligned}
\left(k_{1}-k_{2}\right)_{\nu} T_{\lambda \mu \nu}^{R,(1),\{A V\} V}=-\frac{i}{4 \pi^{2}} \epsilon_{\nu \lambda \mu \xi}\left(k_{1}-k_{2}\right)_{\nu}\left(k_{3}-k_{1}\right)_{\xi} \int_{0}^{1} d x_{1} \int_{0}^{x_{1}} d x_{2} e^{-M^{2} / M_{c}^{2}}(7.5) \\
\left(k_{3}-k_{2}\right)_{\lambda} T_{\lambda \mu \nu}^{R,(1),\{A V\} V}=\frac{i}{4 \pi^{2}} \epsilon_{\nu \lambda \mu \xi}\left(k_{1}-k_{2}\right)_{\lambda}\left(k_{3}-k_{1}\right)_{\xi} \int_{0}^{1} d x_{1} \int_{0}^{x_{1}} d x_{2} e^{-M^{2} / M_{c}^{2}}
\end{aligned}
$$

Taking the UV cut-off $M_{c}$ to be infinity, i.e., $M_{c} \rightarrow \infty$, the Ward identities become

$$
\begin{aligned}
\left(k_{3}-k_{1}\right)_{\mu} T_{\lambda \mu \nu}^{R,(1),\{A V\} V} & =0 \\
\left(k_{1}-k_{2}\right)_{\nu} T_{\lambda \mu \nu}^{R,(1),\{A V\} V} & =-\frac{i}{8 \pi^{2}} \epsilon_{\nu \lambda \mu \xi}\left(k_{1}-k_{2}\right)_{\nu}\left(k_{3}-k_{1}\right)_{\xi} \\
\left(k_{3}-k_{2}\right)_{\lambda} T_{\lambda \mu \nu}^{R,(1),\{A V\} V} & =\frac{i}{8 \pi^{2}} \epsilon_{\nu \lambda \mu \xi}\left(k_{1}-k_{2}\right)_{\lambda}\left(k_{3}-k_{1}\right)_{\xi}
\end{aligned}
$$

which indicates that when taking the IR cut-off scale $\mu_{s}=0$ and UV cut-off scale $M_{c} \rightarrow \infty$ in the loop regularization, and grouping the gamma with the Lorentz indices of one of vector currents and axial-vector current to reduce the number of gamma in the trace, the anomalies appear in the two grouped currents, the remaining vector current is conserved.

To have two vectors conserved, the physical amplitude should be redefined as

$$
\tilde{T}_{\lambda \mu \nu}^{(1),\{A V\} V}\left(k_{3}-k_{1}, k_{1}-k_{2}\right)=T_{\lambda \mu \nu}^{R,(1),\{A V\} V}\left(k_{3}-k_{1}, k_{1}-k_{2}\right)-T_{\lambda \mu \nu}^{(1),\{A V\} V}(0)
$$

with

$$
T_{\lambda \mu \nu}^{(1),\{A V\} V}(0)=-\frac{i}{8 \pi^{2}} \epsilon_{\nu \lambda \mu \xi}\left(k_{3}-k_{1}\right)_{\xi}
$$

Then the redefined amplitude satisfies the following Ward identities

$$
\begin{aligned}
\left(k_{3}-k_{1}\right)_{\mu} \tilde{T}_{\lambda \mu \nu}^{(1),\{A V\} V} & =0 \\
\left(k_{1}-k_{2}\right)_{\nu} \tilde{T}_{\lambda \mu \nu}^{(1),\{A V\} V} & =0 \\
\left(k_{3}-k_{2}\right)_{\lambda} \tilde{T}_{\lambda \mu \nu}^{(1),\{A V\} V} & =\frac{i}{4 \pi^{2}} \epsilon_{\nu \lambda \mu \xi}\left(k_{1}-k_{2}\right)_{\lambda}\left(k_{3}-k_{1}\right)_{\xi}
\end{aligned}
$$

By including the cross diagram, we get the standard form of Ward identity anomaly

$$
\begin{aligned}
\left(k_{3}-k_{1}\right)_{\mu} \tilde{T}_{\lambda \mu \nu}^{(1),\{A V\} V} & =0 \\
\left(k_{1}-k_{2}\right)_{\nu} \tilde{T}_{\lambda \mu \nu}^{(1),\{A V\} V} & =0 \\
\left(k_{3}-k_{2}\right)_{\lambda} \tilde{T}_{\lambda \mu \nu}^{(1),\{A V\} V} & =-\frac{i}{2 \pi^{2}} \epsilon_{\mu \nu \alpha \beta}\left(k_{3}-k_{1}\right)_{\alpha}\left(k_{1}-k_{2}\right)_{\beta}
\end{aligned}
$$

It is seen that in the treatment with relation (3.13), the IR scale $\mu_{s}$ plays the same role as the other two cases. Comparing with the treatment with relation (3.11), one observes 
that the Ward identity anomalies always appear in the currents with the Lorentz indices classified in the group which was treated with relation (3.9), in the perturbative calculations of triangle anomaly, how the Ward identity anomalies depend on the treatments for the trace of gamma matrices. After comparing all the three treatments for the trace of gamma matrices in the loop regularization, it is not difficult to arrive at the conclusion: In order to obtain a unique and right solution for the Ward identity anomaly, one shall make the most general treatment for the trace of gamma matrices, namely, by using the definition of $\gamma_{5}$.

\section{Calculation of anomaly by using Pauli-Villars regularization}

In Pauli-Villars regularization, the regularized version of (17.43) and (7.44) are given by

$$
\begin{aligned}
T_{L, \lambda \mu \nu}^{R,(1),\{A V\} V}= & -4 \sum_{i=0}^{2} C_{i} \int \frac{d^{4} k}{(2 \pi)^{4}}\left\{\left\{g_{\nu \lambda} \epsilon_{\alpha \beta \mu \xi}\left(k+k_{1}\right)_{\alpha}\left(k+k_{2}\right)_{\beta}\left(k+k_{3}\right)_{\xi}\right.\right. \\
& +\epsilon_{\nu \beta \mu \xi}\left(k+k_{1}\right)_{\beta}\left(k+k_{2}\right)_{\lambda}\left(k+k_{3}\right)_{\xi}+\epsilon_{\lambda \beta \mu \xi}\left(k+k_{1}\right)_{\beta}\left(k+k_{2}\right)_{\nu}\left(k+k_{3}\right)_{\xi} \\
& \left.+\epsilon_{\lambda \alpha \nu \beta}\left(k+k_{1}\right)_{\beta}\left(k+k_{2}\right)_{\alpha}\left(k+k_{3}\right)_{\mu}+\epsilon_{\lambda \alpha \nu \xi}\left(k+k_{1}\right)_{\mu}\left(k+k_{2}\right)_{\alpha}\left(k+k_{3}\right)_{\xi}\right\} \\
& \times\left[\frac{1}{\left[\left(k+k_{1}\right)^{2}-m_{i}^{2}\right]\left[\left(k+k_{2}\right)^{2}-m_{i}^{2}\right]\left[\left(k+k_{3}\right)^{2}-m_{i}^{2}\right]}\right] \\
& -\frac{\left(k+k_{2}\right)_{\alpha}}{2}\left[\frac{(k \alpha \mu \mu}{\left[\left(k+k_{2}\right)^{2}-m_{i}^{2}\right]\left[\left(k+k_{3}\right)^{2}-m_{i}^{2}\right]}\right. \\
& \left.\left.\quad+\frac{\left(k+k_{2}\right)_{\alpha}}{\left[\left(k+k_{2}\right)^{2}-m_{i}^{2}\right]\left[\left(k+k_{1}\right)^{2}-m_{i}^{2}\right]}\right]\right\} \\
T_{C, \lambda \mu \nu}^{R,(1),\{A V\} V}= & -2 \epsilon_{\lambda \alpha \nu \mu} \sum_{i=0}^{2} C_{i} \int \frac{d^{4} k}{(2 \pi)^{4}} \frac{1}{\left[\left(k+k_{1}\right)^{2}-m_{i}^{2}\right]\left[\left(k+k_{2}\right)^{2}-m_{i}^{2}\right]\left[\left(k+k_{3}\right)^{2}-m_{i}^{2}\right]} \\
& \times\left\{2 m_{i}^{2}\left(k+k_{3}\right)_{\alpha}-\left[4 m_{i}^{2}-\left(k_{3}-k_{1}\right)^{2}\right]\left(k+k_{2}\right)_{\alpha}+2 m_{i}^{2}\left(k+k_{1}\right)_{\alpha}\right\}
\end{aligned}
$$

Following the same evaluation as done in the previous subsection, we obtain the following Ward identities

$$
\begin{aligned}
\left(k_{3}-k_{1}\right)_{\mu} T_{\lambda \mu \nu}^{R,(1),\{A V\} V} & =0 \\
\left(k_{1}-k_{2}\right)_{\nu} T_{\lambda \mu \nu}^{R,(1),\{A V\} V} & =-\frac{i}{8 \pi^{2}} \sum_{i=0}^{2} C_{i} \epsilon_{\nu \lambda \mu \xi}\left(k_{1}-k_{2}\right)_{\nu}\left(k_{3}-k_{1}\right)_{\xi}=0 \\
\left(k_{3}-k_{2}\right)_{\lambda} T_{\lambda \mu \nu}^{R,(1),\{A V\} V} & =-\frac{i}{8 \pi^{2}} \sum_{i=0}^{2} C_{i} \epsilon_{\nu \lambda \mu \xi}\left(k_{1}-k_{2}\right)_{\xi}\left(k_{3}-k_{1}\right)_{\lambda}+\sum_{i=0}^{2} C_{i}\left[2 m_{i} T_{\mu \nu}^{(1), P V V}\left(m_{i}\right)\right] \\
& =\sum_{i=0}^{2} C_{i}\left[2 m_{i} T_{\mu \nu}^{(1), P V V}\left(m_{i}\right)\right]
\end{aligned}
$$

which shows that in the treatment with relation (3.13), the Ward identities are the same as the ones in the treatment with relation (3.11). Although the final results are the same 
in these two treatments, while the anomalies in the two vector currents are cancelled by the heavy regulator field in latter case. Here the remaining vector current is conserved automatically.

Taking the masses of regulator fermions to be infinity large, we have

$$
\sum_{i=0}^{2} C_{i}\left[2 m_{i} T_{\mu \nu}^{(1), P V V}\left(m_{i}\right)\right]=-\frac{i}{4 \pi^{2}} \epsilon_{\mu \nu \alpha \beta}\left(k_{3}-k_{1}\right)_{\alpha}\left(k_{1}-k_{2}\right)_{\beta}
$$

Here the source of anomaly remains arising from the heavy regulator fermion loops.

\section{Calculation of anomaly by using dimensional regularization}

By using eq. (3.13), the regularized version for the parallel part of the amplitude can be written as

$$
\begin{aligned}
T_{\|, \lambda \mu \nu}^{R,(1),\{A V\} V}= & T_{\|, 0 \lambda \mu \nu}^{R,(1),\{A V\} V}+T_{\|,-2 \lambda \mu \nu}^{R,(1),\{A V\} V} \\
T_{\|, 0 \lambda \mu \nu}^{R,(1),\{A V\} V}= & 2 \int_{0}^{1} d x_{1} \int_{0}^{x_{1}} d x_{2}\left\{\epsilon_{\mu \lambda \nu \beta}\left(k_{3}+k_{1}-2 k_{2}\right)_{\beta} \frac{i}{16 \pi^{2}}\left[-\ln M^{2}\right]\right\} \\
& -2 \epsilon_{\mu \alpha \nu \lambda}\left\{\int_{0}^{1} d x\left(-\Delta_{2}+k_{2}\right)_{\alpha} \frac{i}{16 \pi^{2}}\left[-\ln M_{2}^{2}\right]\right. \\
& \left.+\int_{0}^{1} d x\left(-\Delta_{1}+k_{2}\right)_{\alpha} \frac{i}{16 \pi^{2}}\left[-\ln M_{1}^{2}\right]\right\} \\
T_{\|,-2 \lambda \mu \nu}^{R,(1),\{A V\} V}= & -4 \int_{0}^{1} d x_{1} \int_{0}^{x_{1}} d x_{2} \\
& \times\left\{-2\left\{\epsilon_{\mu \beta \nu \xi}\left[\left(-\Delta+k_{1}\right)_{\beta}\left(-\Delta+k_{2}\right)_{\lambda}\left(-\Delta+k_{3}\right)_{\xi}\right]\right.\right. \\
& +\epsilon_{\mu \beta \lambda \xi}\left[\left(-\Delta+k_{1}\right)_{\beta}\left(-\Delta+k_{2}\right)_{\nu}\left(-\Delta+k_{3}\right)_{\xi}\right] \\
& +\epsilon_{\nu \alpha \lambda \beta}\left[\left(-\Delta+k_{1}\right)_{\beta}\left(-\Delta+k_{2}\right)_{\alpha}\left(-\Delta+k_{3}\right)_{\mu}\right] \\
& \left.+\epsilon_{\nu \alpha \lambda \xi}\left[\left(-\Delta+k_{1}\right)_{\mu}\left(-\Delta+k_{3}\right)_{\xi}\left(-\Delta+k_{2}\right)_{\alpha}\right]\right\} \\
& \left.+\epsilon_{\lambda \alpha \nu \mu}\left\{\left(k_{3}-k_{1}\right)^{2}\left(-\Delta+k_{2}\right)_{\alpha}\right\}\right\} \frac{-i}{32 \pi^{2}} \frac{1}{M^{2}} \\
& +\frac{i}{32 \pi^{2}} \epsilon_{\mu \lambda \nu \beta}\left(k_{3}+k_{1}-2 k_{2}\right)_{\beta}
\end{aligned}
$$

From the definitions of $\Delta$ and $\Delta_{i}$ and the relations given in App.A. and App.C., we obtain the following Ward identities

$$
\begin{aligned}
\left(k_{3}-k_{1}\right)_{\mu} T_{\|, \lambda \mu \nu}^{(1),\{A V\} V} & =-\frac{4}{16} \frac{i}{4 \pi^{2}} \epsilon_{\mu \nu \lambda \alpha}\left(k_{3}-k_{1}\right)_{\mu}\left(k_{1}-k_{2}\right)_{\alpha} \\
\left(k_{1}-k_{2}\right)_{\nu} T_{\|, \lambda \mu \nu}^{(1),\{A V\} V} & =-\frac{10}{16} \frac{i}{4 \pi^{2}} \epsilon_{\nu \lambda \mu \xi}\left(k_{1}-k_{2}\right)_{\nu}\left(k_{3}-k_{1}\right)_{\xi} \\
\left(k_{3}-k_{2}\right)_{\lambda} T_{\|, \lambda \mu \nu}^{(1),\{A V\} V} & =\frac{10}{16} \frac{i}{4 \pi^{2}} \epsilon_{\nu \lambda \mu \xi}\left(k_{1}-k_{2}\right)_{\lambda}\left(k_{3}-k_{1}\right)_{\xi}
\end{aligned}
$$


Combining the result in eq. (6.9), we have

$$
\begin{aligned}
\left(k_{3}-k_{1}\right)_{\mu} T_{\lambda \mu \nu}^{(1),\{A V\} V} & =-\frac{14}{24} \frac{i}{4 \pi^{2}} \epsilon_{\mu \nu \lambda \alpha}\left(k_{3}-k_{1}\right)_{\mu}\left(k_{1}-k_{2}\right)_{\alpha} \\
\left(k_{1}-k_{2}\right)_{\nu} T_{\lambda \mu \nu}^{(1),\{A V\} V} & =-\frac{7}{24} \frac{i}{4 \pi^{2}} \epsilon_{\nu \lambda \mu \xi}\left(k_{1}-k_{2}\right)_{\nu}\left(k_{3}-k_{1}\right)_{\xi} \\
\left(k_{3}-k_{2}\right)_{\lambda} T_{\lambda \mu \nu}^{(1),\{A V\} V} & =\frac{31}{24} \frac{i}{4 \pi^{2}} \epsilon_{\nu \lambda \mu \xi}\left(k_{1}-k_{2}\right)_{\lambda}\left(k_{3}-k_{1}\right)_{\xi}
\end{aligned}
$$

By including the cross diagram, we arrive at the final results

$$
\begin{aligned}
\left(k_{3}-k_{1}\right)_{\mu} T_{\lambda \mu \nu}^{R,\{A V\} V} & =-\frac{14}{12} \frac{i}{4 \pi^{2}} \epsilon_{\nu \lambda \mu \xi}\left(k_{1}-k_{2}\right)_{\xi}\left(k_{3}-k_{1}\right)_{\mu} \\
\left(k_{1}-k_{2}\right)_{\nu} T_{\lambda \mu \nu}^{R,\{A V\} V} & =-\frac{7}{12} \frac{i}{4 \pi^{2}} \epsilon_{\nu \lambda \mu \xi}\left(k_{1}-k_{2}\right)_{\nu}\left(k_{3}-k_{1}\right)_{\xi} \\
\left(k_{3}-k_{2}\right)_{\lambda} T_{\lambda \mu \nu}^{R,\{A V\} V} & =\frac{31}{12} \frac{i}{4 \pi^{2}} \epsilon_{\nu \lambda \mu \xi}\left(k_{1}-k_{2}\right)_{\lambda}\left(k_{3}-k_{1}\right)_{\xi}
\end{aligned}
$$

which indicates that when treating the gamma trace with relation (3.13) in the dimensional regularization, anomalies remain appearing in all the three currents. But the explicit forms of anomaly are different from the case in which the gamma trace is treated with relation (3.11) and all the amplitudes are different from the conclusion based on the relation (3.11). In addition, in the present case, the magnitudes of all the three currents are different while in the latter case the magnitudes of the two vector currents are same. To require two vectors conserved, the physical amplitude should be redefined as

$$
\tilde{T}_{\lambda \mu \nu}^{R,\{A V\} V}\left(k_{3}-k_{1}, k_{1}-k_{2}\right)=T_{\lambda \mu \nu}^{R,\{A V\} V}\left(k_{3}-k_{1}, k_{1}-k_{2}\right)-T_{\lambda \mu \nu}^{\{A V\} V}(0)
$$

with

$$
T_{\lambda \mu \nu}^{\{A V\} V}(0)=-\frac{7}{12} \frac{i}{4 \pi^{2}} \epsilon_{\nu \lambda \mu \xi}\left(k_{3}-k_{1}\right)_{\xi}-\frac{14}{12} \frac{i}{4 \pi^{2}} \epsilon_{\nu \lambda \mu \xi}\left(k_{1}-k_{2}\right)_{\xi}
$$

With the above redefinition, one then gets the standard form of anomaly

$$
\begin{aligned}
& \left(k_{3}-k_{1}\right)_{\mu} \tilde{T}_{\lambda \mu \nu}^{R,\{A V\} V}=0 \\
& \left(k_{1}-k_{2}\right)_{\nu} \tilde{T}_{\lambda \mu \nu}^{R,\{A V\} V}=0 \\
& \left(k_{3}-k_{2}\right)_{\lambda} \tilde{T}_{\lambda \mu \nu}^{R,\{A V\} V}=-\frac{i}{2 \pi^{2}} \epsilon_{\mu \nu \alpha \beta}\left(k_{3}-k_{1}\right)_{\alpha}\left(k_{1}-k_{2}\right)_{\beta}
\end{aligned}
$$

In conclusion, in the treatment of the gamma trace with relation (3.13) in dimensional regularization, the anomalies still appear in both vector and axial-vector Ward identities. But the anomalies in the three Ward identities are different from that in treatment with relation (3.11), so that the redefinition of the amplitude is also different although both treatments can make the vector currents conserved and the axial-vector Ward identity is violated by quantum corrections. 


\section{Calculation of anomaly with relation (3.11) in massive QED}

\section{Calculation of anomaly in the loop regularization}

As in the massless case, we first consider the case that the trace of gamma matrix is calculated by using the relation (3.10) and repeating the same calculations as done for massless QED, we yield

$$
\begin{aligned}
T_{0 \lambda \mu \nu}^{R,(1), A\{V V\}}= & 2 \int_{0}^{1} d x_{1} \int_{0}^{x_{1}} d x_{2}\left\{\epsilon_{\mu \lambda \nu \beta}\left(k_{2}+k_{3}-2 k_{1}\right)_{\beta} I_{0}^{R}\left(x_{i}, \mu\right)\right\} \\
& -2 \epsilon_{\mu \alpha \nu \lambda}\left\{\int_{0}^{1} d x\left(-\Delta_{1}+k_{1}\right)_{\alpha} I_{0}^{R}\left(x, \mu_{1}\right)+\int_{0}^{1} d x\left(-\Delta_{3}+k_{1}\right)_{\alpha} I_{0}^{R}\left(x, \mu_{3}\right)\right\} \\
T_{-2 \lambda \mu \nu}^{R,(1), A\{V V\}}= & -4 \int_{0}^{1} d x_{1} \int_{0}^{x_{1}} d x_{2} \\
& \times\left\{-2\left\{\epsilon_{\nu \beta \lambda \xi}\left[\left(-\Delta_{1}+k_{1}\right)_{\mu}\left(-\Delta_{1}+k_{2}\right)_{\beta}\left(-\Delta_{1}+k_{3}\right)_{\xi}\right]\right.\right. \\
& +\epsilon_{\mu \beta \lambda \alpha}\left[\left(-\Delta_{1}+k_{3}\right)_{\alpha}\left(-\Delta_{1}+k_{2}\right)_{\beta}\left(-\Delta_{1}+k_{1}\right)_{\nu}\right] \\
& +\epsilon_{\mu \alpha \nu \beta}\left[\left(-\Delta_{1}+k_{1}\right)_{\alpha}\left(-\Delta_{1}+k_{2}\right)_{\beta}\left(-\Delta_{1}+k_{3}\right)_{\lambda}\right] \\
& \left.+\epsilon_{\mu \alpha \nu \beta}\left[\left(-\Delta_{1}+k_{1}\right)_{\alpha}\left(-\Delta_{1}+k_{3}\right)_{\beta}\left(-\Delta_{1}+k_{2}\right)_{\lambda}\right]\right\} \\
& +2 \epsilon_{\mu \nu \alpha \lambda}\left\{m^{2}\left(k_{2}+k_{3}-2 k_{1}\right)_{\alpha}\right\} \\
& \left.+\epsilon_{\mu \nu \alpha \lambda}\left\{\left(k_{2}-k_{3}\right)^{2}\left(-\Delta_{1}+k_{1}\right)_{\alpha}\right\}\right\} I_{-2}^{R}\left(x_{i}, \mu\right)
\end{aligned}
$$

The Ward identity for vector current is given by

$$
\begin{aligned}
\left(k_{1}-k_{2}\right)_{\nu} T_{\lambda \mu \nu}^{R,(1), A\{V V\}}= & -2 \epsilon_{\mu \nu \lambda \beta}\left(k_{1}-k_{2}\right)_{\nu}\left(k_{3}-k_{1}\right)_{\beta} \\
& \times\left\{4\left(k_{3}-k_{1}\right) \cdot\left(k_{1}-k_{2}\right)\left[I_{-2,(11)}+I_{-2,(20)}-I_{-2,10}\right]\right. \\
& -4\left(k_{2}-k_{1}\right)^{2}\left[I_{-2,(02)}+I_{-2,(11)}-I_{-2,(01)}\right] \\
& \left.-4 m^{2} I_{-2,(00)}+2\left(k_{2}-k_{3}\right)^{2} I_{-2,(10)}\right\} \\
& -2 \epsilon_{\mu \nu \lambda \beta}\left(k_{1}-k_{2}\right)_{\nu}\left(k_{3}-k_{1}\right)_{\beta} I_{0,(00)}
\end{aligned}
$$

By adopting the relations given in App. A, we have

$$
\begin{aligned}
\left(k_{1}-k_{2}\right)_{\nu} T_{\lambda \mu \nu}^{R,(1), A\{V V\}}= & -2 \epsilon_{\mu \nu \lambda \beta}\left(k_{1}-k_{2}\right)_{\nu}\left(k_{3}-k_{1}\right)_{\beta} \\
& \times\left\{\left\{\frac{i}{16 \pi^{2}}\left[e^{-\left(m^{2}+\mu_{s}^{2}\right) / M_{c}^{2}} \int_{0}^{1} d x_{1} \int_{0}^{x_{1}} d x_{2} e^{-M^{2} / M_{c}^{2}}-\frac{1}{2} Y\left((p+q)^{2}, q^{2}\right)\right]\right.\right. \\
& \left.-q^{2} I_{-2,(10)}-p^{2} I_{-2,(01)}+2\left(m^{2}+\mu_{s}^{2}\right) I_{-2,(00)}\right\} \\
& -4 m^{2} I_{-2,(00)}+2(p+q)^{2} I_{-2,(10)}
\end{aligned}
$$




$$
\begin{aligned}
& +4\left\{p^{2} I_{-2,(01)}-p \cdot q I_{-2,(10)}\right. \\
& +\frac{i}{64 \pi^{2}} e^{-\left(m^{2}+\mu_{s}^{2}\right) / M_{c}^{2}} \int_{0}^{1} d x_{1} \int_{0}^{x_{1}} d x_{2} e^{-M^{2} / M_{c}^{2}}+\frac{m^{2}+\mu_{s}^{2}}{2} I_{-2,(00)} \\
& \left.\left.-\frac{q^{2}}{4} I_{-2,(10)}-\frac{3 p^{2}}{4} I_{-2,(01)}+\frac{i}{128 \pi^{2}} Y\left((p+q)^{2}, q^{2}\right)-\frac{p^{2}}{2} I_{-2,(10)}\right\}\right\}
\end{aligned}
$$

With simple algebraic evaluation, the above expression is simplified to be

$$
\begin{aligned}
\left(k_{1}-k_{2}\right)_{\nu} T_{\lambda \mu \nu}^{R,(1), A\{V V\}}= & 8 \mu_{s}^{2} \epsilon_{\mu \lambda \alpha \beta}\left(k_{1}-k_{2}\right)_{\alpha}\left(k_{3}-k_{1}\right)_{\beta} I_{-2,(00)} \\
& +\frac{i}{4 \pi^{2}} \epsilon_{\mu \lambda \alpha \beta}\left(k_{1}-k_{2}\right)_{\alpha}\left(k_{3}-k_{1}\right)_{\beta} \\
& \times e^{-\left(m^{2}+\mu_{s}^{2}\right) / M_{c}^{2}} \int_{0}^{1} d x_{1} \int_{0}^{x_{1}} d x_{2} e^{-M^{2} / M_{c}^{2}}
\end{aligned}
$$

which is different from (4.13) and the two superficially logarithemically divergent integrals cancel each other. Similarly, one has

$$
\begin{aligned}
\left(k_{3}-k_{1}\right)_{\mu} T_{\lambda \mu \nu}^{R,(1), A\{V V\}}= & -8 \mu_{s}^{2} \epsilon_{\nu \lambda \alpha \beta}\left(k_{1}-k_{2}\right)_{\alpha}\left(k_{3}-k_{1}\right)_{\beta} I_{-2,(00)} \\
& -\frac{i}{4 \pi^{2}} \epsilon_{\nu \lambda \alpha \beta}\left(k_{1}-k_{2}\right)_{\alpha}\left(k_{3}-k_{1}\right)_{\beta} \\
& \times e^{-\left(m^{2}+\mu_{s}^{2}\right) / M_{c}^{2}} \int_{0}^{1} d x_{1} \int_{0}^{x_{1}} d x_{2} e^{-M^{2} / M_{c}^{2}}
\end{aligned}
$$

which is different from (4.14).

For the Ward identity of axial-vector current, we have

$$
\begin{aligned}
\left(k_{3}-k_{2}\right)_{\lambda} T_{\lambda \mu \nu}^{R,(1), A\{V V\}}= & 4 \epsilon_{\mu \nu \alpha \beta}\left(k_{2}-k_{1}\right)_{\alpha}\left(k_{3}-k_{1}\right)_{\beta} \\
& \times\left\{2\left(k_{2}-k_{1}\right) \cdot\left(k_{3}-k_{2}\right)\left[I_{-2,(20)}-I_{-2,(02)}+I_{-2,(01)}-I_{-2,(10)}\right]\right. \\
+ & 2\left(k_{3}-k_{2}\right)^{2}\left[I_{-2,(10)}-I_{-2,(20)}+I_{-2,(11)}\right] \\
+ & \left.4 m^{2} I_{-2,(00)}-\left(k_{2}-k_{3}\right)^{2}\left[I_{-2,(01)}+I_{-2,(10)}\right]\right\} \\
& -2 \epsilon_{\mu \nu \lambda \beta}\left(k_{1}-k_{2}\right)_{\lambda}\left(k_{3}-k_{1}\right)_{\beta} I_{0,(00)}^{\prime} \\
& -2 \epsilon_{\mu \nu \lambda \beta}\left(k_{1}-k_{2}\right)_{\lambda}\left(k_{3}-k_{1}\right)_{\beta} I_{0,(00)} \\
= & 16 m^{2} \epsilon_{\mu \nu \alpha \beta}\left(k_{2}-k_{1}\right)_{\alpha}\left(k_{3}-k_{1}\right)_{\beta} I_{-2,(00)}
\end{aligned}
$$

which is different from (4.18).

In comparison with eq.(4.21), we obtain the following relation between two amplitudes

$$
\left(k_{3}-k_{2}\right)_{\lambda} T_{\lambda \mu \nu}^{R,(1), A\{V V\}}=2 m T_{\mu \nu}^{R,(1), P V V}
$$

which shows that the classical axial-vector Ward identity is still preserved by quantum corrections. 
To further evaluate the Ward identity of vector current, we take the limit $M_{c}^{2} \rightarrow \infty$ allowed for the renormalizable massive QED. Under this limit, the Ward identities are simplified to be

$$
\begin{aligned}
\left(k_{3}-k_{1}\right)_{\mu} T_{\lambda \mu \nu}^{R,(1), A\{V V\}=} & -8 \mu_{s}^{2} \epsilon_{\nu \lambda \alpha \beta}\left(k_{1}-k_{2}\right)_{\alpha}\left(k_{3}-k_{1}\right)_{\beta} I_{-2,(00)} \\
& -\frac{i}{8 \pi^{2}} \epsilon_{\nu \lambda \alpha \beta}\left(k_{1}-k_{2}\right)_{\alpha}\left(k_{3}-k_{1}\right)_{\beta} \\
\left(k_{1}-k_{2}\right)_{\nu} T_{\lambda \mu \nu}^{R,(1), A\{V V\}=}= & 8 \mu_{s}^{2} \epsilon_{\mu \lambda \alpha \beta}\left(k_{1}-k_{2}\right)_{\alpha}\left(k_{3}-k_{1}\right)_{\beta} I_{-2,(00)} \\
& +\frac{i}{8 \pi^{2}} \epsilon_{\mu \lambda \alpha \beta}\left(k_{1}-k_{2}\right)_{\alpha}\left(k_{3}-k_{1}\right)_{\beta} \\
\left(k_{3}-k_{2}\right)_{\lambda} T_{\lambda \mu \nu}^{R,(1), A\{V V\}=}= & 2 m T_{\mu \nu}^{R,(1), P V V}=16 m^{2} \epsilon_{\mu \nu \alpha \beta}\left(k_{1}-k_{2}\right)_{\alpha}\left(k_{3}-k_{1}\right)_{\beta} I_{-2,(00)}
\end{aligned}
$$

Firstly, consider the case $\mu_{s} \neq 0$. Similar to the case in massless QED, if taking the two vector states state to be massless with the conditions $p^{2}=q^{2}=0$ and the axial-vector state $(p+q)^{2}$ to be soft, we have

$$
\begin{aligned}
& \left(k_{3}-k_{1}\right)_{\mu} T_{\lambda \mu \nu}^{R,(1), A\{V V\}}=0 \\
& \left(k_{1}-k_{2}\right)_{\nu} T_{\lambda \mu \nu}^{R,(1), A\{V V\}}=0 \\
& \left(k_{3}-k_{2}\right)_{\lambda} T_{\lambda \mu \nu}^{R,(1), A\{V V\}}=2 m T_{\mu \nu}^{R,(1), P V V}
\end{aligned}
$$

which indicates that the Ward identities are well preserved in this case.

In the case that $\mu_{s} \gg m$, the axial-vector current also approaches to anomaly free

$$
\begin{aligned}
\left(k_{3}-k_{2}\right)_{\lambda} T_{\lambda \mu \nu}^{R,(1), A\{V V\}} & =2 m T_{\mu \nu}^{(1), P V V} \\
& =-\frac{i}{4 \pi^{2}} \frac{m^{2}}{\mu_{s}^{2}+m^{2}} \epsilon_{\mu \nu \alpha \beta}\left(k_{3}-k_{1}\right)_{\alpha}\left(k_{1}-k_{2}\right)_{\beta} \rightarrow 0
\end{aligned}
$$

Generally speaking, in the limit $p^{2}, q^{2},(p+q)^{2}, m^{2} \ll \mu_{s}^{2} \ll M_{c}^{2} \rightarrow \infty$, the Ward identities for both currents are preserved in the quantum corrections. Obviously, to arrive at this conclusion, the IR cut-off scale $\mu_{s}$ plays an important role.

Secondly, consider the case that $\mu_{s}^{2}=0$, the relevant Ward identities have the following forms

$$
\begin{aligned}
\left(k_{3}-k_{1}\right)_{\mu} T_{\lambda \mu \nu}^{R,(1), A\{V V\}} & =-\frac{i}{4 \pi^{2}} \epsilon_{\nu \lambda \alpha \beta}\left(k_{1}-k_{2}\right)_{\alpha}\left(k_{3}-k_{1}\right)_{\beta} \int_{0}^{1} d x_{1} \int_{0}^{x_{1}} d x_{2} e^{-\left(m^{2}+M^{2}\right) / \lambda f_{i}^{2}} \\
\left(k_{1}-k_{2}\right)_{\nu} T_{\lambda \mu \nu}^{R,(1), A\{V V\}} & =\frac{i}{4 \pi^{2}} \epsilon_{\mu \lambda \alpha \beta}\left(k_{1}-k_{2}\right)_{\alpha}\left(k_{3}-k_{1}\right)_{\beta} \int_{0}^{1} d x_{1} \int_{0}^{x_{1}} d x_{2} e^{-\left(m^{2}+M^{2}\right) / M^{2}}(7.93 \\
\left(k_{3}-k_{2}\right)_{\lambda} T_{\lambda \mu \nu}^{R,(1), A\{V V\}} & =2 m T_{\mu \nu}^{R,(1), P V V}
\end{aligned}
$$


which indicates that in the loop regularization with $\mu_{s}=0$ the vector current is no longer conserved, while the axial-vector Ward identity is still preserved.

Taking the limit $M_{c}^{2} \rightarrow \infty$, the above results are simplified to be

$$
\begin{aligned}
\left(k_{3}-k_{1}\right)_{\mu} T_{\lambda \mu \nu}^{R,(1), A\{V V\}} & =-\frac{i}{4 \pi^{2}} \epsilon_{\nu \lambda \alpha \beta}\left(k_{1}-k_{2}\right)_{\alpha}\left(k_{3}-k_{1}\right)_{\beta} \\
\left(k_{1}-k_{2}\right)_{\nu} T_{\lambda \mu \nu}^{R,(1), A\{V V\}} & =\frac{i}{4 \pi^{2}} \epsilon_{\mu \lambda \alpha \beta}\left(k_{1}-k_{2}\right)_{\alpha}\left(k_{3}-k_{1}\right)_{\beta} \\
\left(k_{3}-k_{2}\right)_{\lambda} T_{\lambda \mu \nu}^{R,(1), A\{V V\}} & =2 m T_{\mu \nu}^{R,(1), P V V}
\end{aligned}
$$

which shows that in this case the well-known anomaly appears in the vector Ward identity.

Again, by using the redefinition (7.17), we get the amplitude which makes the vector current conserved and anomaly lives in the axial-vector Ward identity

$$
\begin{aligned}
& \left(k_{3}-k_{1}\right)_{\mu} \widetilde{T}_{\lambda \mu \nu}^{(1), A\{V V\}}=0 \\
& \left(k_{1}-k_{2}\right)_{\nu} \widetilde{T}_{\lambda \mu \nu}^{(1), A\{V V\}}=0 \\
& \left(k_{3}-k_{2}\right)_{\lambda} \widetilde{T}_{\lambda \mu \nu}^{(1), A\{V V\}}=(I(m, p, q)-1) \frac{i}{4 \pi^{2}} \epsilon_{\mu \nu \alpha \beta}\left(k_{3}-k_{1}\right)_{\alpha}\left(k_{1}-k_{2}\right)_{\beta}
\end{aligned}
$$

where the integral $I(m, p, q)$ is defined by (4.36).

In the case that the external vector states are massless with conditions: $p^{2}=0, q^{2}=0$ and the axial-vector state be soft, using (4.37), we have

$$
\left(k_{3}-k_{2}\right)_{\lambda} \widetilde{T}_{\lambda \mu \nu}^{(1), A\{V V\}}=0
$$

Namely both vector and axial-vector receive no contribution from quantum corrections for massive QED with general conditions $m^{2} \gg p^{2}, q^{2}, p \cdot q$.

In an alternative case that $p^{2}=0, q^{2}=0$, and $(p+q)^{2} \gg m^{2}$, by using (4.39), the axial-vector gets anomaly.

By including the cross diagrams, the Ward identities are given by

$$
\begin{aligned}
& \left(k_{3}-k_{1}\right)_{\mu} \widetilde{T}_{\lambda \mu \nu}^{A\{V V\}}=0 \\
& \left(k_{1}-k_{2}\right)_{\nu} \widetilde{T}_{\lambda \mu \nu}^{A\{V V\}}=0 \\
& \left(k_{3}-k_{2}\right)_{\lambda} \widetilde{T}_{\lambda \mu \nu}^{A\{V V\}}=2 m T_{\mu \nu}^{R, P V V}-\frac{i}{2 \pi^{2}} \epsilon_{\mu \nu \alpha \beta}\left(k_{3}-k_{1}\right)_{\alpha}\left(k_{1}-k_{2}\right)_{\beta}
\end{aligned}
$$

with the operator forms

$$
\begin{aligned}
\partial_{\mu} V_{\mu}(x) & =0 \\
\partial_{\mu} A_{\mu}(x) & =2 i m P(x)+\frac{e^{2}}{8 \pi^{2}} F^{\mu \nu}(x) \widetilde{F}_{\mu \nu}(x)
\end{aligned}
$$


which is the standard form of triangle anomaly.

It is seen that for massive QED the IR scale $\mu_{s}^{2}$ plays the same role for two treatments.

\section{Calculation of anomaly by using Pauli-Villars regularization}

With a similar evaluation as the massless case, the regularized versions are given by

$$
\begin{aligned}
T_{L, \lambda \mu \nu}^{R,(1), A\{V V\}}= & -4 \sum_{i=0}^{2} C_{i} \int \frac{d^{4} k}{(2 \pi)^{4}}\left\{\left\{g_{\mu \nu} \epsilon_{\alpha \beta \lambda \xi}\left(k+k_{1}\right)_{\alpha}\left(k+k_{2}\right)_{\beta}\left(k+k_{3}\right)_{\xi}\right.\right. \\
& -\epsilon_{\nu \beta \lambda \xi}\left(k+k_{1}\right)_{\mu}\left(k+k_{2}\right)_{\beta}\left(k+k_{3}\right)_{\xi}-\epsilon_{\mu \beta \lambda \xi}\left(k+k_{1}\right)_{\nu}\left(k+k_{2}\right)_{\beta}\left(k+k_{3}\right)_{\xi} \\
& \left.-\epsilon_{\mu \alpha \nu \beta}\left(k+k_{1}\right)_{\alpha}\left(k+k_{3}\right)_{\beta}\left(k+k_{2}\right)_{\lambda}-\epsilon_{\mu \alpha \nu \xi}\left(k+k_{1}\right)_{\alpha}\left(k+k_{2}\right)_{\xi}\left(k+k_{3}\right)_{\lambda}\right\} \\
& \times\left[\frac{1}{\left[\left(k+k_{1}\right)^{2}-m_{i}^{2}\right]\left[\left(k+k_{2}\right)^{2}-m_{i}^{2}\right]\left[\left(k+k_{3}\right)^{2}-m_{i}^{2}\right]}\right] \\
& +\frac{\epsilon_{\mu \alpha \nu \lambda}}{2}\left[\frac{\left(k+k_{1}\right)_{\alpha}}{\left[\left(k+k_{1}\right)^{2}-m_{i}^{2}\right]\left[\left(k+k_{2}\right)^{2}-m_{i}^{2}\right]}\right. \\
& \left.\left.+\frac{\left(k+k_{1}\right)_{\alpha}}{\left[\left(k+k_{1}\right)^{2}-m_{i}^{2}\right]\left[\left(k+k_{3}\right)^{2}-m_{i}^{2}\right]}\right]\right\} \\
T_{C, \lambda \mu \nu}^{(1), A\{V V\}}= & -2 \epsilon_{\mu \nu \alpha \lambda} \sum_{i=0}^{2} C_{i} \int \frac{d^{4} k}{(2 \pi)^{4}} \\
& \times \frac{2 m_{i}^{2}\left(k+k_{2}\right)_{\alpha}-\left[4 m_{i}^{2}-\left(k_{2}-k_{3}\right)^{2}\right]\left(k+k_{1}\right)_{\alpha}+2 m_{i}^{2}\left(k+k_{3}\right)_{\alpha}}{\left[\left(k+k_{1}\right)^{2}-m_{i}^{2}\right]\left[\left(k+k_{2}\right)^{2}-m_{i}^{2}\right]\left[\left(k+k_{3}\right)^{2}-m_{i}^{2}\right]}
\end{aligned}
$$

with the initial condition $c_{0}=1$ and $m_{0}=m$. After adopting Feynman parametrization and shifting the integration variables, we have

$$
\begin{aligned}
T_{\lambda \mu \nu}^{R,(1), A\{V V\}}= & T_{0 \lambda \mu \nu}^{R,(1), A\{V V\}}+T_{-2 \lambda \mu \nu}^{R,(1), A\{V V\}} \\
T_{0 \lambda \mu \nu}^{R,(1), A\{V V\}}= & -2 \sum_{i=0}^{2} C_{i} \int_{0}^{1} d x_{1} \int_{0}^{x_{1}} d x_{2}\left\{\epsilon_{\mu \lambda \nu \beta}\left(k_{2}+k_{3}-2 k_{1}\right)_{\beta} \int \frac{d^{4} k}{(2 \pi)^{2}} \frac{k^{2}}{\left[k^{2}-M_{i}^{2}\right]^{3}}\right\} \\
& -2 \epsilon_{\mu \alpha \nu \lambda}\left\{\sum_{i=0}^{2} C_{i} \int_{0}^{1} d x\left(-\Delta_{2}+k_{1}\right)_{\alpha} \int \frac{d^{4} k}{(2 \pi)^{4}} \frac{1}{\left[k^{2}-M_{1 i}^{2}\right]^{2}}\right. \\
& \left.+\sum_{i=0}^{2} C_{i} \int_{0}^{1} d x\left(-\Delta_{3}+k_{1}\right)_{\alpha} \int \frac{d^{4} k}{(2 \pi)^{4}} \frac{1}{\left[k^{2}-M_{3 i}^{2}\right]^{2}}\right\} \\
T_{-2 \lambda \mu \nu}^{R,(1), A\{V V\}=} & 4 \sum_{i=0}^{2} C_{i} \int_{0}^{1} d x_{1} \int_{0}^{x_{1}} d x_{2} \\
& \times\left\{2 \left\{\epsilon_{\mu \beta \lambda \xi}\left[\left(-\Delta_{1}+k_{1}\right)_{\nu}\left(-\Delta_{1}+k_{2}\right)_{\beta}\left(-\Delta_{1}+k_{3}\right)_{\xi}\right]\right.\right. \\
& +\epsilon_{\nu \beta \lambda \alpha}\left[\left(-\Delta_{1}+k_{3}\right)_{\alpha}\left(-\Delta_{1}+k_{2}\right)_{\beta}\left(-\Delta_{1}+k_{1}\right)_{\mu}\right] \\
& +\epsilon_{\mu \alpha \nu \beta}\left[\left(-\Delta_{1}+k_{1}\right)_{\alpha}\left(-\Delta_{1}+k_{2}\right)_{\beta}\left(-\Delta_{1}+k_{3}\right)_{\lambda}\right]
\end{aligned}
$$




$$
\begin{aligned}
& \left.+\epsilon_{\mu \alpha \nu \beta}\left[\left(-\Delta_{1}+k_{1}\right)_{\alpha}\left(-\Delta_{1}+k_{3}\right)_{\beta}\left(-\Delta_{1}+k_{2}\right)_{\lambda}\right]\right\} \\
& -2 \epsilon_{\mu \nu \alpha \lambda} m_{i}^{2}\left(k_{2}+k_{3}-2 k_{1}\right)_{\alpha} \\
& \left.-\epsilon_{\mu \nu \alpha \lambda}\left(k_{2}-k_{3}\right)^{2}\left(-\Delta_{1}+k_{1}\right)_{\alpha}\right\} \int \frac{d^{4} k}{(2 \pi)^{4}} \frac{1}{\left[k^{2}-M_{i}^{2}\right]^{3}}
\end{aligned}
$$

After some algebra and again adopting the useful relations presented in App. B and App. A, we have

$$
\begin{aligned}
\left(k_{3}-k_{1}\right)_{\mu} T_{\lambda \mu \nu}^{R,(1), A\{V V\}} & =\sum_{i=0}^{2} C_{i}\left(\frac{-i}{8 \pi^{2}}\right) \epsilon_{\lambda \alpha \beta}\left(k_{1}-k_{2}\right)_{\alpha}\left(k_{3}-k_{1}\right)_{\beta}=0 \\
\left(k_{1}-k_{2}\right)_{\nu} T_{\lambda \mu \nu}^{R,(1), A\{V V\}} & =\sum_{i=0}^{2} C_{i}\left(\frac{i}{8 \pi^{2}}\right) \epsilon_{\mu \lambda \alpha \beta}\left(k_{1}-k_{2}\right)_{\alpha}\left(k_{3}-k_{1}\right)_{\beta}=0 \\
\left(k_{3}-k_{2}\right)_{\lambda} T_{\lambda \mu \nu}^{R,(1), A\{V V\}} & =\sum_{i=0}^{2} C_{i}\left[2 m_{i} T_{\mu \nu}^{(1), P V V}\left(m_{i}\right)\right]
\end{aligned}
$$

which is the same as the massless case that the vector ward identity is automatically preserved. For axial-vector current, using (5.11), we have

$$
\left(k_{3}-k_{2}\right)_{\lambda} \widetilde{T}_{\lambda \mu \nu}^{(1), A\{V V\}}=(I(m, p, q)-1) \frac{i}{4 \pi^{2}} \epsilon_{\mu \nu \alpha \beta}\left(k_{3}-k_{1}\right)_{\alpha}\left(k_{1}-k_{2}\right)_{\beta}
$$

For the external states with conditions: $p^{2}=0, q^{2}=0$, and $(p+q)^{2}=2 p \cdot q$ being soft with $m^{2} \gg p \cdot q$, one has from eq.(4.37) both vector and axial-vector become anomaly free in massive QED.

Considering the alternative case that $p^{2}=0, q^{2}=0$, and $(p+q)^{2} \gg m^{2}$ and eq.(4.39), then the axial-vector gets anomaly. It is seen that Pauli-Villars scheme leads to the same conclusions as loop regularization with $\mu_{s}=0$.

\section{Calculation of anomaly by using dimensional regularization}

As seen from eq. (6.6), in the dimensional regularization the amplitude $T_{\lambda \mu \nu}^{(1), A\{V V\}}$ can be decomposed in terms of two parts $T_{\|, \lambda \mu \nu}^{(1), A\{V V\}}$ and $T_{\perp, \lambda \mu \nu}^{(1), A\{V V\}}$. For the part $T_{\perp, \lambda \mu \nu}^{(1), A\{V V\}}$, the result is the same as the one given in eq.(6.9). To evaluate the part $T_{\|, \lambda \mu \nu}^{(1), A\{V V\}}$, we can adopt the integration relations presented in App. C. and consider the relations given in App. A.. The final results with the cross diagram are found to be

$$
\begin{aligned}
\left(k_{3}-k_{1}\right)_{\mu} T_{\lambda \mu \nu}^{R, A\{V V\}} & =\frac{14}{24} \frac{-i}{4 \pi^{2}} \epsilon_{\nu \lambda \alpha \beta}\left(k_{1}-k_{2}\right)_{\alpha}\left(k_{3}-k_{1}\right)_{\beta} \\
\left(k_{1}-k_{2}\right)_{\nu} T_{\lambda \mu \nu}^{R, A\{V V\}} & =\frac{14}{24} \frac{i}{4 \pi^{2}} \epsilon_{\mu \lambda \alpha \beta}\left(k_{1}-k_{2}\right)_{\alpha}\left(k_{3}-k_{1}\right)_{\beta} \\
\left(k_{3}-k_{2}\right)_{\lambda} T_{\lambda \mu \nu}^{R, A\{V V\}} & =32 m^{2} \epsilon_{\alpha \mu \beta \nu}\left(k_{3}-k_{1}\right)_{\alpha}\left(k_{1}-k_{2}\right)_{\beta} I_{-2,(00)}+\frac{20}{24} \frac{i}{4 \pi^{2}} \epsilon_{\mu \nu \lambda \beta}\left(k_{1}-k_{2}\right)_{\lambda}\left(k_{3}-k_{1}\right)_{\beta}
\end{aligned}
$$


Consider the result eq.(4.21), the three Ward identities can be rewritten as

$$
\begin{aligned}
\left(k_{3}-k_{1}\right)_{\mu} T_{\lambda \mu \nu}^{R, A\{V V\}} & =\frac{14}{24} \frac{-i}{4 \pi^{2}} \epsilon_{\nu \lambda \alpha \beta}\left(k_{1}-k_{2}\right)_{\alpha}\left(k_{3}-k_{1}\right)_{\beta} \\
\left(k_{1}-k_{2}\right)_{\nu} T_{\lambda \mu \nu}^{R, A\{V V\}} & =\frac{14}{24} \frac{i}{4 \pi^{2}} \epsilon_{\mu \lambda \alpha \beta}\left(k_{1}-k_{2}\right)_{\alpha}\left(k_{3}-k_{1}\right)_{\beta} \\
\left(k_{3}-k_{2}\right)_{\lambda} T_{\lambda \mu \nu}^{R, A\{V V\}} & =2 m T_{\mu \nu}^{R, P V V}+\frac{20}{24} \frac{i}{4 \pi^{2}} \epsilon_{\mu \nu \lambda \beta}\left(k_{1}-k_{2}\right)_{\lambda}\left(k_{3}-k_{1}\right)_{\beta}
\end{aligned}
$$

To keep the conservation of vector current, making a similar redefinition for the physical amplitude as in eq.(17.38), we then obtain for the redefined amplitude the standard form of Ward identities

$$
\begin{aligned}
& \left(k_{3}-k_{1}\right)_{\mu} \widetilde{T}_{\lambda \mu \nu}^{A\{V V\}}\left(k_{3}-k_{1}, k_{1}-k_{2}\right)=0 \\
& \left(k_{1}-k_{2}\right)_{\nu} \widetilde{T}_{\lambda \mu \nu}^{A\{V V\}}\left(k_{3}-k_{1}, k_{1}-k_{2}\right)=0 \\
& \left(k_{3}-k_{2}\right)_{\nu} \widetilde{T}_{\lambda \mu \nu}^{A\{V V\}}\left(k_{3}-k_{1}, k_{1}-k_{2}\right)=2 m T_{\mu \nu}^{R, P V V}-\frac{i}{2 \pi^{2}} \epsilon_{\mu \nu \alpha \beta}\left(k_{3}-k_{1}\right)_{\alpha}\left(k_{1}-k_{2}\right)(\widetilde{\beta} .11
\end{aligned}
$$

The discussions given in the previous sections are applicable to the above results.

\section{Calculation of anomaly with relation (3.13) in massive QED}

\section{Calculation of anomaly in the loop regularization}

In this subsection, we will investigate the anomaly by evaluate the trace of gamma matix by using the relation (3.13). Repeating the calculations done in the massless case, the massive AVV amplitude can be expressed as

$$
\begin{aligned}
T_{\lambda \mu \nu}^{R,(1),\{A V\} V}= & T_{0 \lambda \mu \nu}^{R,(1),\{A V\} V}+T_{-2 \lambda \mu \nu}^{R,(1),\{A V\} V} \\
T_{0 \lambda \mu \nu}^{R,(1),\{A V\} V}= & 2 \int_{0}^{1} d x_{1} \int_{0}^{x_{1}} d x_{2}\left\{\epsilon_{\mu \lambda \nu \beta}\left(k_{3}+k_{1}-2 k_{2}\right)_{\beta} I_{0}^{R}\left(x_{i}, \mu\right)\right\} \\
& -2 \epsilon_{\mu \alpha \nu \lambda}\left\{\int_{0}^{1} d x\left(-\Delta_{2}+k_{2}\right)_{\alpha} I_{0}^{R}\left(x, \mu_{2}\right)+\int_{0}^{1} d x\left(-\Delta_{1}+k_{2}\right)_{\alpha} I_{0}^{R}\left(x, \mu_{1}\right)\right\} \\
T_{-2 \lambda \mu \nu}^{R,(1),\{A V\} V}= & -4 \int_{0}^{1} d x_{1} \int_{0}^{x_{1}} d x_{2} \\
& \times\left\{-2\left\{\epsilon_{\mu \beta \nu \xi}\left[\left(-\Delta+k_{1}\right)_{\beta}\left(-\Delta+k_{2}\right)_{\lambda}\left(-\Delta+k_{3}\right)_{\xi}\right]\right.\right. \\
& +\epsilon_{\mu \beta \lambda \xi}\left[\left(-\Delta+k_{1}\right)_{\beta}\left(-\Delta+k_{2}\right)_{\nu}\left(-\Delta+k_{3}\right)_{\xi}\right] \\
& +\epsilon_{\nu \alpha \lambda \beta}\left[\left(-\Delta+k_{1}\right)_{\beta}\left(-\Delta+k_{2}\right)_{\alpha}\left(-\Delta+k_{3}\right)_{\mu}\right] \\
& \left.+\epsilon_{\nu \alpha \lambda \xi}\left[\left(-\Delta+k_{1}\right)_{\mu}\left(-\Delta+k_{3}\right)_{\xi}\left(-\Delta+k_{2}\right)_{\alpha}\right]\right\}
\end{aligned}
$$




$$
\begin{aligned}
& +\epsilon_{\lambda \alpha \nu \mu}\left[\left(k_{3}-k_{1}\right)^{2}\left(-\Delta+k_{2}\right)_{\alpha}\right] \\
& \left.+2 m^{2} \epsilon_{\lambda \nu \mu \alpha}\left(k_{3}-k_{1}\right)_{\alpha}\right\} I_{-2}^{R}\left(x_{i}, \mu\right)
\end{aligned}
$$

For three Ward identities, by using the definitions of $\Delta$ and $\Delta_{i}$, we have

$$
\begin{aligned}
& \left(k_{1}-k_{2}\right)_{\nu} T_{\lambda \mu \nu}^{R,(1),\{A V\} V}=-2 \epsilon_{\nu \lambda \mu \xi}\left(k_{1}-k_{2}\right)_{\nu}\left(k_{3}-k_{1}\right)_{\xi} \\
& \times\left\{4 \left[\left(k_{1}-k_{2}\right)^{2} I_{-2,(01)}-\left(k_{1}-k_{2}\right)^{2} I_{-2,(02)}\right.\right. \\
& \left.+\left(k_{3}-k_{1}\right) \cdot\left(k_{1}-k_{2}\right) I_{-2,(11)}\right]+2\left(k_{3}-k_{1}\right)^{2} I_{-2,(10)} \\
& \left.+2 m^{2} \epsilon_{\lambda \nu \mu \alpha}\left(k_{1}-k_{2}\right)_{\nu}\left(k_{3}-k_{1}\right)_{\alpha} I_{-2,(00)}\right\} \\
& -2 \epsilon_{\nu \lambda \mu \xi}\left(k_{1}-k_{2}\right)_{\nu}\left(k_{3}-k_{1}\right)_{\xi}\left\{I_{0,(00)}+\frac{i}{32 \pi^{2}} Y\left((p+q)^{2}, q^{2}\right)\right\} \\
& \left(k_{3}-k_{2}\right)_{\lambda} T_{\lambda \mu \nu}^{R,(1),\{A V\} V}=-2 \epsilon_{\nu \lambda \mu \xi}\left(k_{1}-k_{2}\right)_{\xi}\left(k_{3}-k_{1}\right)_{\lambda} \\
& \times\left\{4 \left[\left(k_{1}-k_{2}\right)^{2} I_{-2,(01)}-\left(k_{1}-k_{2}\right)^{2} I_{-2,(02)}\right.\right. \\
& +\left(k_{1}-k_{2}\right) \cdot\left(k_{3}-k_{1}\right) I_{-2,(01)}-\left(k_{1}-k_{2}\right) \cdot\left(k_{3}-k_{1}\right) I_{-2,(02)} \\
& \left.+\left(k_{1}-k_{2}\right) \cdot\left(k_{3}-k_{1}\right) I_{-2,(11)}+\left(k_{3}-k_{1}\right)^{2} I_{-2,(11)}\right] \\
& +2\left[\left(k_{3}-k_{1}\right)^{2} I_{-2,(00)}-\left(k_{3}-k_{1}\right)^{2} I_{-2,(01)}-\left(k_{3}-k_{1}\right)^{2} I_{-2,(10)}\right] \\
& \left.++2 m^{2} \epsilon_{\lambda \nu \mu \alpha}\left(k_{1}-k_{2}\right)_{\lambda}\left(k_{3}-k_{1}\right)_{\alpha}\right\} \\
& -2 \epsilon_{\nu \lambda \mu \xi}\left(k_{1}-k_{2}\right)_{\xi}\left(k_{3}-k_{1}\right)_{\lambda} I_{0,(00)}^{\prime} \\
& \left(k_{3}-k_{1}\right)_{\mu} T_{\lambda \mu \nu}^{R,(1),\{A V\} V}=-2 \epsilon_{\nu \lambda \mu \xi}\left(k_{3}-k_{1}\right)_{\mu}\left(k_{1}-k_{2}\right)_{\xi} \\
& \times\left\{-4\left[-\left(k_{1}-k_{2}\right) \cdot\left(k_{3}-k_{1}\right) I_{-2,(01)}+\left(k_{1}-k_{2}\right) \cdot\left(k_{3}-k_{1}\right) I_{-2,(02)}\right.\right. \\
& +2\left(k_{1}-k_{2}\right) \cdot\left(k_{3}-k_{1}\right) I_{-2,(11)}+\left(k_{3}-k_{1}\right)^{2} I_{-2,(10)}-\left(k_{3}-k_{1}\right)^{2} I_{-2,(11)} \\
& \left.-2\left(k_{3}-k_{1}\right)^{2} I_{-2,(20)}+\left(k_{3}-k_{1}\right)^{2} I_{-2,(10)}\right] \\
& \left.+2\left[\left(k_{3}-k_{1}\right)^{2} I_{-2,(00)}-\left(k_{3}-k_{1}\right)^{2} I_{-2,(01)}\right]\right\} \\
& -2 \epsilon_{\nu \lambda \mu \xi}\left(k_{3}-k_{1}\right)_{\mu}\left(k_{1}-k_{2}\right)_{\xi}\left\{I_{0,(00)}+\frac{i}{32 \pi^{2}} Y\left((p+q)^{2}, q^{2}\right)\right\} \\
& -2 \epsilon_{\nu \lambda \mu \xi}\left(k_{3}-k_{1}\right)_{\mu}\left(k_{1}-k_{2}\right)_{\xi} I_{0,(00)}^{\prime}
\end{aligned}
$$

Using the massive relations given in App.A., we can rewrite the above Ward identities as

$$
\begin{aligned}
\left(k_{3}-k_{1}\right)_{\mu} T_{\lambda \mu \nu}^{R,(1),\{A V\} V}= & 0 \\
\left(k_{1}-k_{2}\right)_{\nu} T_{\lambda \mu \nu}^{R,(1),\{A V\} V}= & -8 \mu_{s}^{2} \epsilon_{\nu \lambda \mu \xi}\left(k_{1}-k_{2}\right)_{\nu}\left(k_{3}-k_{1}\right)_{\xi} I_{-2,(00)} \\
& -\frac{i}{4 \pi^{2}} \epsilon_{\nu \lambda \mu \xi}\left(k_{1}-k_{2}\right)_{\nu}\left(k_{3}-k_{1}\right)_{\xi} e^{-\left(m^{2}+\mu_{s}^{2}\right) / M_{c}^{2}} \int_{0}^{1} d x_{1} \int_{0}^{x_{1}} d x_{2} e^{-M^{2} / M_{c}^{2}}
\end{aligned}
$$




$$
\begin{aligned}
\left(k_{3}-k_{2}\right)_{\lambda} T_{\lambda \mu \nu}^{R,(1),\{A V\} V}= & -8 \mu_{s}^{2} \epsilon_{\nu \lambda \mu \xi}\left(k_{1}-k_{2}\right)_{\xi}\left(k_{3}-k_{1}\right)_{\lambda} I_{-2,(00)} \\
& -\frac{i}{4 \pi^{2}} \epsilon_{\nu \lambda \mu \xi}\left(k_{1}-k_{2}\right)_{\xi}\left(k_{3}-k_{1}\right)_{\lambda} e^{-\left(m^{2}+\mu_{s}^{2}\right) / M_{c}^{2}} \int_{0}^{1} d x_{1} \int_{0}^{x_{1}} d x_{2} e^{-M^{2} / M_{c}^{2}} \\
& +16 m^{2} \epsilon_{\nu \lambda \mu \xi}\left(k_{1}-k_{2}\right)_{\nu}\left(k_{3}-k_{1}\right)_{\xi} I_{-2,(00)}
\end{aligned}
$$

It is noticed that in the treatment with the trace relation (3.13), one of the vectors is conserved. This result is not the same as the one considered in the previous subsection, where the relation (3.11) was used in the calculation and anomalies appear in two vector Ward identities.

By considering the PVV amplitude result (4.21), the axial-vector Ward identity can be reexpressed as:

$$
\begin{aligned}
\left(k_{3}-k_{2}\right)_{\lambda} T_{\lambda \mu \nu}^{R,(1),\{A V\} V}= & -8 \mu_{s}^{2} \epsilon_{\nu \lambda \mu \xi}\left(k_{1}-k_{2}\right)_{\xi}\left(k_{3}-k_{1}\right)_{\lambda} I_{-2,(00)} \\
& -\frac{i}{4 \pi^{2}} \epsilon_{\nu \lambda \mu \xi}\left(k_{1}-k_{2}\right)_{\xi}\left(k_{3}-k_{1}\right)_{\lambda} e^{-\left(m^{2}+\mu_{s}^{2}\right) / M_{c}^{2}} \int_{0}^{1} d x_{1} \int_{0}^{x_{1}} d x_{2} e^{-M^{2} / M_{c}^{2}} \\
& +2 m T_{\mu \nu}^{R,(1), P V V}
\end{aligned}
$$

As the massive QED is renormalizable, we take the limit $M_{c} \rightarrow \infty$, the Ward identities become

$$
\begin{aligned}
\left(k_{3}-k_{1}\right)_{\mu} T_{\lambda \mu \nu}^{R,(1),\{A V\} V}= & 0 \\
\left(k_{1}-k_{2}\right)_{\nu} T_{\lambda \mu \nu}^{R,(1),\{A V\} V=}= & -8 \mu_{s}^{2} \epsilon_{\nu \lambda \mu \xi}\left(k_{1}-k_{2}\right)_{\nu}\left(k_{3}-k_{1}\right)_{\xi} I_{-2,(00)} \\
& -\frac{i}{4 \pi^{2}} \epsilon_{\nu \lambda \mu \xi}\left(k_{1}-k_{2}\right)_{\nu}\left(k_{3}-k_{1}\right)_{\xi} \\
\left(k_{3}-k_{2}\right)_{\lambda} T_{\lambda \mu \nu}^{R,(1),\{A V\} V=}= & -8 \mu_{s}^{2} \epsilon_{\nu \lambda \mu \xi}\left(k_{1}-k_{2}\right)_{\xi}\left(k_{3}-k_{1}\right)_{\lambda} I_{-2,(00)} \\
& -\frac{i}{4 \pi^{2}} \epsilon_{\nu \lambda \mu \xi}\left(k_{1}-k_{2}\right)_{\xi}\left(k_{3}-k_{1}\right)_{\lambda} \\
& +2 m T_{\mu \nu}^{R,(1), P V V}
\end{aligned}
$$

As in section III.B, we now consider two cases. Firstly, consider the case that $\mu_{s} \neq 0$, taking the external vector states be massless and on their mass shell $p^{2}=\left(k_{1}-k_{2}\right)^{2}=$ $0, q^{2}=\left(k_{3}-k_{1}\right)^{2}=0$ and the axial-vector state be soft, we then have

$$
\begin{aligned}
& \left(k_{3}-k_{1}\right)_{\mu} T_{\lambda \mu \nu}^{R,(1),\{A V\} V}=0 \\
& \left(k_{1}-k_{2}\right)_{\nu} T_{\lambda \mu \nu}^{R,(1),\{A V\} V}=0 \\
& \left(k_{3}-k_{2}\right)_{\lambda} T_{\lambda \mu \nu}^{R,(1),\{A V\} V}=2 m T_{\mu \nu}^{R,(1), P V V}
\end{aligned}
$$


which means that with the above conditions both the vector and axial-vector Ward identities are preserved. In fact, in the case $\mu_{s} \gg m$, the quantum corrections to the axial-vector current Ward identity also approach to zero as shown in eq. (17.91).

In conclusion, in the treatment with relation (3.13), the anomaly free conditions are the same as the case in the treatment with relation (3.11).

We now come to consider the case with $\mu_{s}=0$, the massive Ward identities eqs.17.121. 7.123) can be rewritten as

$$
\begin{aligned}
\left(k_{3}-k_{1}\right)_{\mu} T_{\lambda \mu \nu}^{R,(1),\{A V\} V}= & 0 \\
\left(k_{1}-k_{2}\right)_{\nu} T_{\lambda \mu \nu}^{R,(1),\{A V\} V}= & -\frac{i}{4 \pi^{2}} \epsilon_{\nu \lambda \mu \xi}\left(k_{1}-k_{2}\right)_{\nu}\left(k_{3}-k_{1}\right)_{\xi} \int_{0}^{1} d x_{1} \int_{0}^{x_{1}} d x_{2} e^{-\left(M^{2}+m^{2}\right) / M_{c}^{2}} \\
\left(k_{3}-k_{2}\right)_{\lambda} T_{\lambda \mu \nu}^{R,(1),\{A V\} V=}= & -\frac{i}{4 \pi^{2}} \epsilon_{\nu \lambda \mu \xi}\left(k_{1}-k_{2}\right)_{\xi}\left(k_{3}-k_{1}\right)_{\lambda} \int_{0}^{1} d x_{1} \int_{0}^{x_{1}} d x_{2} e^{-\left(M^{2}+m^{2}\right) / M_{c}^{2}} \\
& +2 m T_{\mu \nu}^{R,(1), P V V}
\end{aligned}
$$

Taking the limit $M_{c} \rightarrow \infty$ since the massive QED is renormalizable, the results can be expressed as

$$
\begin{aligned}
\left(k_{3}-k_{1}\right)_{\mu} T_{\lambda \mu \nu}^{R,(1),\{A V\} V} & =0 \\
\left(k_{1}-k_{2}\right)_{\nu} T_{\lambda \mu \nu}^{R,(1),\{A V\} V} & =-\frac{i}{8 \pi^{2}} \epsilon_{\nu \lambda \mu \xi}\left(k_{1}-k_{2}\right)_{\nu}\left(k_{3}-k_{1}\right)_{\xi} \\
\left(k_{3}-k_{2}\right)_{\lambda} T_{\lambda \mu \nu}^{R,(1),\{A V\} V} & =2 m T_{\mu \nu}^{R,(1), P V V}-\frac{i}{8 \pi^{2}} \epsilon_{\nu \lambda \mu \xi}\left(k_{1}-k_{2}\right)_{\xi}\left(k_{3}-k_{1}\right)_{\lambda}
\end{aligned}
$$

which are the Ward identities when the trace of gamma matrices is manipulated with relation (3.13) where the gamma matrices of the Lorentz indices of the axial-vector current and one of the vector currents are grouped to reduce the number of gamma matrices.

Again to keep both the vector currents conserved, we can use eq. (17.60) to redefine the physical amplitude. The Ward identities for the redefined amplitude become

$$
\begin{aligned}
& \left(k_{3}-k_{1}\right)_{\mu} \tilde{T}_{\lambda \mu \nu}^{(1),\{A V\} V}=0 \\
& \left(k_{1}-k_{2}\right)_{\nu} \tilde{T}_{\lambda \mu \nu}^{(1),\{A V\} V}=0 \\
& \left(k_{3}-k_{2}\right)_{\lambda} \tilde{T}_{\lambda \mu \nu}^{(1),\{A V\} V}=(I(m, p, q)-1) \frac{i}{4 \pi^{2}} \epsilon_{\mu \nu \alpha \beta}\left(k_{3}-k_{1}\right)_{\alpha}\left(k_{1}-k_{2}\right)_{\beta}
\end{aligned}
$$

where the function $I(m, p, q)$ was defined in eq. (4.36). In the case that the external vector are massless with conditions: $p^{2}=0, q^{2}=0$, and the axial-vector state $(p+q)^{2}=2 p \cdot q$ is soft and using eq. (4.39), we have

$$
\left(k_{3}-k_{2}\right)_{\lambda} \widetilde{T}_{\lambda \mu \nu}^{(1),\{A V\} V}=0
$$


Namely both vector and axial-vector become anomaly free for massive QED with the general conditions $m^{2} \gg p^{2}, q^{2},(p+q)^{2}$.

While in an alternative case that $p^{2}=0, q^{2}=0$ and $(p+q)^{2} \gg m^{2}$, we have

$$
\begin{aligned}
\left(k_{3}-k_{1}\right)_{\mu} \widetilde{T}_{\lambda \mu \nu}^{(1)\{A V\} V} & =0 \\
\left(k_{1}-k_{2}\right)_{\nu} \widetilde{T}_{\lambda \mu \nu}^{(1),\{A V\} V} & =0 \\
\left(k_{3}-k_{2}\right)_{\lambda} \widetilde{T}_{\lambda \mu \nu}^{(1),\{A V\} V} & =2 m T_{\mu \nu}^{R,(1), P V V}-\frac{i}{4 \pi^{2}} \epsilon_{\mu \nu \alpha \beta}\left(k_{3}-k_{1}\right)_{\alpha}\left(k_{1}-k_{2}\right)_{\beta}
\end{aligned}
$$

which can be regarded as a consistent check to the massless QED at $\mu_{s}=0$. In this case, the divergence of the axial-vector current gets anomaly.

When including the cross diagrams, the Ward identities are given by

$$
\begin{aligned}
& \left(k_{3}-k_{1}\right)_{\mu} \widetilde{T}_{\lambda \mu \nu}^{\{A V\} V}=0 \\
& \left(k_{1}-k_{2}\right)_{\nu} \widetilde{T}_{\lambda \mu \nu}^{\{A V\} V}=0 \\
& \left(k_{3}-k_{2}\right)_{\lambda} \widetilde{T}_{\lambda \mu \nu}^{\{A V\} V}=2 m T_{\mu \nu}^{R, P V V}-\frac{i}{2 \pi^{2}} \epsilon_{\mu \nu \alpha \beta}\left(k_{3}-k_{1}\right)_{\alpha}\left(k_{1}-k_{2}\right)_{\beta}
\end{aligned}
$$

\section{Calculation of anomaly by using Pauli-Villars regularization}

In Pauli-Villars regularization, the regularized version of (17.43) and (7.44) have the following forms

$$
\begin{aligned}
T_{L, \lambda \mu \nu}^{R,(1),\{A V\} V}= & -4 \sum_{i=0}^{2} C_{i} \int \frac{d^{4} k}{(2 \pi)^{4}}\left\{\left\{g_{\nu \lambda} \epsilon_{\alpha \beta \mu \xi}\left(k+k_{1}\right)_{\alpha}\left(k+k_{2}\right)_{\beta}\left(k+k_{3}\right)_{\xi}\right.\right. \\
& +\epsilon_{\nu \beta \mu \xi}\left(k+k_{1}\right)_{\beta}\left(k+k_{2}\right)_{\lambda}\left(k+k_{3}\right)_{\xi}+\epsilon_{\lambda \beta \mu \xi}\left(k+k_{1}\right)_{\beta}\left(k+k_{2}\right)_{\nu}\left(k+k_{3}\right)_{\xi} \\
& \left.+\epsilon_{\lambda \alpha \nu \beta}\left(k+k_{1}\right)_{\beta}\left(k+k_{2}\right)_{\alpha}\left(k+k_{3}\right)_{\mu}+\epsilon_{\lambda \alpha \nu \xi}\left(k+k_{1}\right)_{\mu}\left(k+k_{2}\right)_{\alpha}\left(k+k_{3}\right)_{\xi}\right\} \\
& \times\left[\frac{1}{\left[\left(k+k_{1}\right)^{2}-m_{i}^{2}\right]\left[\left(k+k_{2}\right)^{2}-m_{i}^{2}\right]\left[\left(k+k_{3}\right)^{2}-m_{i}^{2}\right]}\right] \\
& -\frac{\left(k+k_{2}\right)_{\alpha}}{2}\left[\frac{\left(k+k_{2}\right)_{\alpha}}{\left[\left(k+k_{2}\right)^{2}-m_{i}^{2}\right]\left[\left(k+k_{3}\right)^{2}-m_{i}^{2}\right]}\right. \\
& \left.\left.\quad+\frac{\left(k+k_{i}\right.}{\left[\left(k+k_{2}\right)^{2}-m_{i}^{2}\right]\left[\left(k+k_{1}\right)^{2}-m_{i}^{2}\right]}\right]\right\} \\
T_{C, \lambda \mu \nu}^{R,(1),\{A V\} V}= & -2 \epsilon_{\lambda \alpha \nu \mu} \sum_{i=0}^{2} C_{i} \int \frac{d^{4} k}{(2 \pi)^{4}} \frac{1}{\left[\left(k+k_{1}\right)^{2}-m_{i}^{2}\right]\left[\left(k+k_{2}\right)^{2}-m_{i}^{2}\right]\left[\left(k+k_{3}\right)^{2}-m_{i}^{2}\right]} \\
& \times\left\{2 m_{i}^{2}\left(k+k_{3}\right)_{\alpha}-\left[4 m_{i}^{2}-\left(k_{3}-k_{1}\right)^{2}\right]\left(k+k_{2}\right)_{\alpha}+2 m_{i}^{2}\left(k+k_{1}\right)_{\alpha}\right\}
\end{aligned}
$$

By shifting the integration variable and making some algebra, we have

$$
T_{\lambda \mu \nu}^{R,(1),\{A V\} V}=T_{0 \lambda \mu \nu}^{R,(1),\{A V\} V}+T_{-2 \lambda \mu \nu}^{R,(1),\{A V\} V}
$$




$$
\begin{aligned}
T_{0 \lambda \mu \nu}^{R,(1),\{A V\} V}= & 2 \sum_{i=0}^{2} C_{i} \int_{0}^{1} d x_{1} \int_{0}^{x_{1}} d x_{2}\left\{\epsilon_{\mu \lambda \nu \beta}\left(k_{3}+k_{1}-2 k_{2}\right)_{\beta} \int \frac{d^{4} k}{(2 \pi)^{4}} \frac{k^{2}}{\left[k^{2}-M_{i}^{2}\right]^{3}}\right\} \\
& -2 \epsilon_{\mu \alpha \nu \lambda}\left\{\sum_{i=0}^{2} C_{i} \int_{0}^{1} d x\left(-\Delta_{2}+k_{2}\right)_{\alpha} \int \frac{d^{4} k}{(2 \pi)^{4}} \frac{1}{\left[k^{2}-M_{2 i}^{2}\right]^{2}}\right. \\
& \left.+\sum_{i=0}^{2} C_{i} \int_{0}^{1} d x\left(-\Delta_{1}+k_{2}\right)_{\alpha} \int \frac{d^{4} k}{(2 \pi)^{4}} \frac{1}{\left[k^{2}-M_{1 i}^{2}\right]^{2}}\right\} \\
T_{-2 \lambda \mu \nu}^{R,(1),\{A V\} V}= & -4 \sum_{i=0}^{2} C_{i} \int_{0}^{1} d x_{1} \int_{0}^{x_{1}} d x_{2} \\
& \times\left\{-2\left\{\epsilon_{\mu \beta \nu \xi}\left[\left(-\Delta+k_{1}\right)_{\beta}\left(-\Delta+k_{2}\right)_{\lambda}\left(-\Delta+k_{3}\right)_{\xi}\right]\right.\right. \\
& +\epsilon_{\mu \beta \lambda \xi}\left[\left(-\Delta+k_{1}\right)_{\beta}\left(-\Delta+k_{2}\right)_{\nu}\left(-\Delta+k_{3}\right)_{\xi}\right] \\
& +\epsilon_{\nu \alpha \lambda \beta}\left[\left(-\Delta+k_{1}\right)_{\beta}\left(-\Delta+k_{2}\right)_{\alpha}\left(-\Delta+k_{3}\right)_{\mu}\right] \\
& \left.+\epsilon_{\nu \alpha \lambda \xi}\left[\left(-\Delta+k_{1}\right)_{\mu}\left(-\Delta+k_{3}\right)_{\xi}\left(-\Delta+k_{2}\right)_{\alpha}\right]\right\} \\
& +\epsilon_{\lambda \alpha \nu \mu}\left[\left(k_{3}-k_{1}\right)^{2}\left(-\Delta+k_{2}\right)_{\alpha}\right] \\
& \left.+2 m^{2} \epsilon_{\lambda \nu \mu \alpha}\left(k_{3}-k_{1}\right)_{\alpha}\right\} \int \frac{d^{4} k}{(2 \pi)^{4}} \frac{1}{\left[k^{2}-M_{i}^{2}\right]^{3}}
\end{aligned}
$$

From the relations given in App.B and also in App.A., we arrive at the following results

$$
\begin{aligned}
\left(k_{3}-k_{1}\right)_{\mu} T_{\lambda \mu \nu}^{(1),\{A V\} V} & =0 \\
\left(k_{1}-k_{2}\right)_{\nu} T_{\lambda \mu \nu}^{R,(1),\{A V\} V} & =-\frac{i}{8 \pi^{2}} \sum_{i=0}^{2} C_{i} \epsilon_{\nu \lambda \mu \xi}\left(k_{1}-k_{2}\right)_{\nu}\left(k_{3}-k_{1}\right)_{\xi}=0 \\
\left(k_{3}-k_{2}\right)_{\lambda} T_{\lambda \mu \nu}^{R,(1),\{A V\} V} & =-\frac{i}{8 \pi^{2}} \sum_{i=0}^{2} C_{i} \epsilon_{\nu \lambda \mu \xi}\left(k_{1}-k_{2}\right)_{\xi}\left(k_{3}-k_{1}\right)_{\lambda}+\sum_{i=0}^{2} C_{i}\left[2 m_{i} T_{\mu \nu}^{(1), P V V}\left(m_{i}\right)\right] \\
& =\sum_{i=0}^{2} C_{i}\left[2 m_{i} T_{\mu \nu}^{(1), P V V}\left(m_{i}\right)\right]
\end{aligned}
$$

Note that as in the massless case, although both two vectors are conserved, while one is caused by the cancellation of the heavy fermion fields and another is conserved automatically. In the infinity mass limit for regulator heavy fermions, we have

$$
\left(k_{3}-k_{2}\right)_{\lambda} T_{\lambda \mu \nu}^{R,(1),\{A V\} V}=2 m T_{\mu \nu}^{(1), P V V}-\frac{i}{4 \pi^{2}} \epsilon_{\mu \nu \alpha \beta}\left(k_{3}-k_{1}\right)_{\alpha}\left(k_{2}-k_{1}\right)_{\beta}
$$

\section{Calculation of anomaly by using dimensional regularization}

In the massive QED, the parallel part of the amplitude is given by

$$
T_{\|, \lambda \mu \nu}^{R,(1),\{A V\} V}=T_{\|, 0 \lambda \mu \nu}^{R,(1),\{A V\} V}+T_{\|,-2 \lambda \mu \nu}^{R,(1),\{A V\} V}
$$




$$
\begin{aligned}
T_{\|, 0 \lambda \mu \nu}^{R,(1),\{A V\} V}= & 2 \int_{0}^{1} d x_{1} \int_{0}^{x_{1}} d x_{2}\left\{\epsilon_{\mu \lambda \nu \beta}\left(k_{3}+k_{1}-2 k_{2}\right)_{\beta} \frac{i}{16 \pi^{2}}\left[-\ln \left(M^{2}+m^{2}\right)\right]\right\} \\
& -2 \epsilon_{\mu \alpha \nu \lambda}\left\{\int_{0}^{1} d x\left(-\Delta_{1}+k_{2}\right)_{\alpha} \frac{i}{16 \pi^{2}}\left[-\ln \left(M_{2}^{2}+m^{2}\right)\right]\right. \\
& \left.+\int_{0}^{1} d x\left(-\Delta+k_{2}\right)_{\alpha} \frac{i}{16 \pi^{2}}\left[-\ln \left(M_{1}^{2}+m^{2}\right)\right]\right\} \\
T_{\|,-2 \lambda \mu \nu}^{R,(1),\{A V\} V}= & -4 \int_{0}^{1} d x_{1} \int_{0}^{x_{1}} d x_{2} \\
& +\left\{-2\left\{\epsilon_{\mu \beta \nu \xi}\left[\left(-\Delta+k_{1}\right)_{\beta}\left(-\Delta+k_{2}\right)_{\lambda}\left(-\Delta+k_{3}\right)_{\xi}\right]\right.\right. \\
& +\epsilon_{\mu \beta \lambda \xi}\left[\left(-\Delta+k_{1}\right)_{\beta}\left(-\Delta+k_{2}\right)_{\nu}\left(-\Delta+k_{3}\right)_{\xi}\right] \\
& +\epsilon_{\nu \alpha \lambda \beta}\left[\left(-\Delta+k_{1}\right)_{\beta}\left(-\Delta+k_{2}\right)_{\alpha}\left(-\Delta+k_{3}\right)_{\mu}\right] \\
& \left.+\epsilon_{\nu \alpha \lambda \xi}\left[\left(-\Delta+k_{1}\right)_{\mu}\left(-\Delta+k_{3}\right)_{\xi}\left(-\Delta+k_{2}\right)_{\alpha}\right]\right\} \\
& +\epsilon_{\lambda \alpha \nu \mu}\left[\left(k_{3}-k_{1}\right)^{2}\left(-\Delta+k_{2}\right)_{\alpha}\right] \\
& \left.+2 m^{2} \epsilon_{\lambda \nu \mu \alpha}\left(k_{3}-k_{1}\right)_{\alpha}\right\} \frac{-i}{32 \pi^{2}} \frac{1}{M^{2}+m^{2}}
\end{aligned}
$$

Using the definitions of $\Delta$ and $\Delta_{i}$, as well as the relations given in App.C. and App.A., we can express the Ward identities as

$$
\begin{aligned}
\left(k_{3}-k_{1}\right)_{\mu} T_{\lambda \mu \nu}^{R,(1),\{A V\} V}= & 0 \\
\left(k_{1}-k_{2}\right)_{\nu} T_{\|, \lambda \mu \nu}^{R,(1),\{A V\} V}= & -\frac{10}{16} \frac{i}{4 \pi^{2}} \epsilon_{\nu \lambda \mu \xi}\left(k_{1}-k_{2}\right)_{\nu}\left(k_{3}-k_{1}\right)_{\xi} \\
\left(k_{3}-k_{2}\right)_{\lambda} T_{\|, \lambda \mu \nu}^{R,(1),\{A V\} V}= & \frac{10}{16} \frac{i}{4 \pi^{2}} \epsilon_{\nu \lambda \mu \xi}\left(k_{1}-k_{2}\right)_{\lambda}\left(k_{3}-k_{1}\right)_{\xi} \\
& +16 m^{2} \epsilon_{\nu \lambda \mu \xi}\left(k_{1}-k_{2}\right)_{\nu}\left(k_{3}-k_{1}\right)_{\xi} I_{-2,(00)}
\end{aligned}
$$

Noticing the result for the PVV diagram and the conclusion of the perpendicular part of the amplitude, we have

$$
\begin{aligned}
\left(k_{3}-k_{1}\right)_{\mu} T_{\lambda \mu \nu}^{(1),\{A V\} V} & =-\frac{14}{24} \frac{i}{4 \pi^{2}} \epsilon_{\mu \nu \lambda \alpha}\left(k_{3}-k_{1}\right)_{\mu}\left(k_{1}-k_{2}\right)_{\alpha} \\
\left(k_{1}-k_{2}\right)_{\nu} T_{\lambda \mu \nu}^{(1),\{A V\} V} & =-\frac{7}{24} \frac{i}{4 \pi^{2}} \epsilon_{\nu \lambda \mu \xi}\left(k_{1}-k_{2}\right)_{\nu}\left(k_{3}-k_{1}\right)_{\xi} \\
\left(k_{3}-k_{2}\right)_{\lambda} T_{\lambda \mu \nu}^{(1),\{A V\} V} & =\frac{31}{24} \frac{i}{4 \pi^{2}} \epsilon_{\nu \lambda \mu \xi}\left(k_{1}-k_{2}\right)_{\lambda}\left(k_{3}-k_{1}\right)_{\xi}+2 m T_{\mu \nu}^{(1), P V V}
\end{aligned}
$$

To keep the vector currents conserved, making again the redefinition (7.76), we then yield the familiar anomaly

$$
\begin{aligned}
& \left(k_{3}-k_{1}\right)_{\mu} \tilde{T}_{\lambda \mu \nu}^{(1),\{A V\} V}=0 \\
& \left(k_{1}-k_{2}\right)_{\nu} \tilde{T}_{\lambda \mu \nu}^{(1),\{A V\} V}=0 \\
& \left(k_{3}-k_{2}\right)_{\lambda} \tilde{T}_{\lambda \mu \nu}^{(1),\{A V\} V}=2 m T_{\mu \nu}^{(1), P V V}-\frac{i}{4 \pi^{2}} \epsilon_{\mu \nu \alpha \beta}\left(k_{3}-k_{1}\right)_{\alpha}\left(k_{1}-k_{2}\right)_{\beta}
\end{aligned}
$$


Including the cross diagram, we arrive at the final results

$$
\begin{aligned}
& \left(k_{3}-k_{1}\right)_{\mu} \tilde{T}_{\lambda \mu \nu}^{\{A V\} V}=0 \\
& \left(k_{1}-k_{2}\right)_{\nu} \tilde{T}_{\lambda \mu \nu}^{\{A V\} V}=0 \\
& \left(k_{3}-k_{2}\right)_{\lambda} \tilde{T}_{\lambda \mu \nu}^{\{A V\} V}=2 m T_{\mu \nu}^{P V V}-\frac{i}{2 \pi^{2}} \epsilon_{\mu \nu \alpha \beta}\left(k_{3}-k_{1}\right)_{\alpha}\left(k_{1}-k_{2}\right)_{\beta}
\end{aligned}
$$

\section{E. On ambiguities of reducing the triangle diagram by acting external momentum}

In some literatures, it is often to demonstrate the calculations of anomaly via the effective PVV amplitude instead of calculating the AVV triangle diagram directly. Here the PVV amplitude is obtained by acting the external momentum of currents on the AVV amplitude. In the following we will show within the framework of massless QED how some ambiguities arise from such kind of approaches in the dimensional regularization and Pauli-Villars scheme. In the loop regularization, as the regularization prescription operates on the irreducible loop integrals (ILIs) that are evaluated from Feynman loop integrals, the action of external momentum on the ILIs does not change the structure of Ward identities, so that there exist no such ambiguities.

\section{The calculation with dimensional regularization}

Following ref. [11], acting the external momentum of axial-vector current $\left(k_{3}-k_{2}\right)_{\lambda}$ on the amplitude (3.8) and using the relation

$$
\left(k_{3}-k_{2}\right)_{\lambda} \gamma_{\lambda} \gamma_{5}=(\not k+\not k / 3) \gamma_{5}+\gamma_{5}(\not k+\not k 2)-\left.2 \gamma_{5} k\right|_{\perp}
$$

we then have

$$
\begin{aligned}
& \left(k_{3}-k_{2}\right)_{\lambda} T_{\lambda \mu \nu}^{(1), A V V}=i \int \frac{d^{n} k}{(2 \pi)^{n}} \operatorname{tr}\left\{\gamma_{5} \frac{1}{(\not k+\not k 2)} \gamma_{\nu} \frac{1}{\left(\not k+\not k_{1}\right)} \gamma_{\mu}\right\} \\
& +i \int \frac{d^{n} k}{(2 \pi)^{n}} \operatorname{tr}\left\{\gamma_{5} \gamma_{\nu} \frac{1}{(\not k+\not k 1)} \gamma_{\mu} \frac{1}{\left(\not k+\not k k_{3}\right)}\right\} \\
& -2 i \int \frac{d^{n} k}{(2 \pi)^{n}} \operatorname{tr}\left\{\gamma_{5} k / \frac{1}{\left(\not k+\not k_{2}\right)} \gamma_{\nu} \frac{1}{\left(\not k+\not k k_{1}\right)} \gamma_{\mu} \frac{1}{\left(\not k+\not k k_{3}\right)}\right\}
\end{aligned}
$$

An explicit calculation shows that the first two terms vanish separately, then the above amplitude is simplified to be

$$
\left.\left(k_{3}-k_{2}\right)_{\lambda} T_{\lambda \mu \nu}^{(1), A V V}=-2 i \int \frac{d^{n} k}{(2 \pi)^{n}} \operatorname{tr}\left\{\gamma_{5} k / \frac{1}{(\not k+\not k 2)} \gamma_{\nu} \frac{1}{(\not k+\not k+k} \gamma_{\mu} \frac{1}{(\not k+\not k+k}\right)\right\}
$$


Using the relation in the dimensional regularization that

$$
\left(\not k_{\perp}\right)^{2}=k_{\perp}^{2} \rightarrow \frac{(d-4)}{d} l^{2}
$$

and considering the results in App.C, we have

$$
\left(k_{3}-k_{2}\right)_{\lambda} T_{\lambda \mu \nu}^{(1), A V V}=-\frac{i}{4 \pi^{2}} \epsilon_{\mu \nu \alpha \beta}\left(k_{3}-k_{1}\right)_{\alpha}\left(k_{1}-k_{2}\right)_{\beta}
$$

Considering the cross diagram, the last form of anomaly is

$$
\left(k_{3}-k_{2}\right)_{\lambda} T_{\lambda \mu \nu}^{A V V}=-\frac{i}{2 \pi^{2}} \epsilon_{\mu \nu \alpha \beta}\left(k_{3}-k_{1}\right)_{\alpha}\left(k_{1}-k_{2}\right)_{\beta}
$$

However, if the external momentum acting on the amplitude is not of the axial-vector current but of vector currents, which results will results will be obtain. To check that, using the relation

$$
\left(k_{3}-k_{1}\right)_{\mu} \gamma_{\mu} \gamma_{5}=(\not k+\not k / 3) \gamma_{5}+\gamma_{5}(\not k+\not k 1)-\left.2 \gamma_{5} k\right|_{\perp}
$$

and moving $\gamma_{5}$ behind $\gamma_{\mu}$ before analytically extending the dimension, namely writing the amplitude as

$$
T_{\lambda \mu \nu}^{(1), A V V}=(-1) \int \frac{d^{4} k}{(2 \pi)^{4}} \operatorname{tr}\left\{\gamma_{\lambda} \frac{i}{\left(\not k+\not k k_{2}\right)} \gamma_{\nu} \frac{i}{\left(\not k+\not k k_{1}\right)} \gamma_{\mu} \gamma_{5} \frac{i}{\left(\not k+\not k k_{3}\right)}\right\}
$$

we then get, along the same calculations as the above, that

$$
\left(k_{3}-k_{1}\right)_{\mu} T_{\lambda \mu \nu}^{(1), A V V}=-\frac{i}{4 \pi^{2}} \epsilon_{\mu \nu \alpha \beta}\left(k_{3}-k_{1}\right)_{\alpha}\left(k_{1}-k_{2}\right)_{\beta}
$$

with the cross diagram, one obtains

$$
\left(k_{3}-k_{1}\right)_{\mu} T_{\lambda \mu \nu}^{A V V}=-\frac{i}{2 \pi^{2}} \epsilon_{\mu \nu \alpha \beta}\left(k_{3}-k_{1}\right)_{\alpha}\left(k_{1}-k_{2}\right)_{\beta}
$$

which shows that in such an evaluation one can not distinguish with currents which is the axial-vector current and where the anomaly lives.

\section{The calculation with Pauli-Villars regularization}

In Pauli-Villars regularization[10], the ambiguity mentioned above becomes manifest. After the action of the external momentum of axial-vector current, we have

$$
\left(k_{3}-k_{2}\right)_{\lambda} T_{\lambda \mu \nu}^{R,(1), A V V}=i \sum_{i=0}^{2} \int \frac{d^{4} k}{(2 \pi)^{4}} \operatorname{tr}\left\{\gamma_{5} \frac{1}{(\not k+\not k 2)-m_{i}} \gamma_{\nu} \frac{1}{(\not k+\not k 1)-m_{i}} \gamma_{\mu}\right\}
$$




$$
\begin{aligned}
& +i \sum_{i=0}^{2} \int \frac{d^{4} k}{(2 \pi)^{4}} \operatorname{tr}\left\{\gamma_{5} \gamma_{\nu} \frac{1}{(\not k+\not k 1)-m_{i}} \gamma_{\mu} \frac{1}{\left(\not k+\not k k_{3}\right)-m_{i}}\right\} \\
& +2 i \sum_{i=0}^{2} m_{i} \int \frac{d^{4} k}{(2 \pi)^{4}}
\end{aligned}
$$

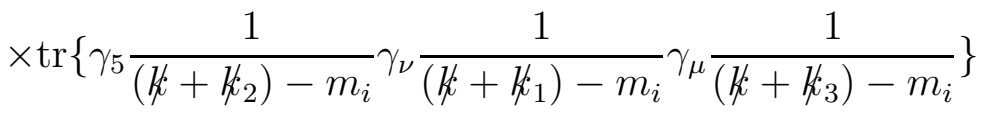

Again the explicit calculation shows that the first two terms are zero separately and we obtain the result

$$
\begin{aligned}
\left(k_{3}-k_{2}\right)_{\lambda} T_{\lambda \mu \nu}^{R,(1), A V V} & =2 \sum_{i=0}^{2} m_{i} T_{\mu \nu}^{(1), P V V}\left(m_{i}\right) \\
& =-\frac{i}{4 \pi^{2}} \epsilon_{\mu \nu \alpha \beta}\left(k_{3}-k_{1}\right)_{\alpha}\left(k_{1}-k_{2}\right)_{\beta}
\end{aligned}
$$

Considering the cross diagram, we have

$$
\begin{aligned}
\left(k_{3}-k_{2}\right)_{\lambda} T_{\lambda \mu \nu}^{R, A V V} & =2 \sum_{i=0}^{2} m_{i} T_{\mu \nu}^{P V V}\left(m_{i}\right) \\
& =-\frac{i}{2 \pi^{2}} \epsilon_{\mu \nu \alpha \beta}\left(k_{3}-k_{1}\right)_{\alpha}\left(k_{1}-k_{2}\right)_{\beta}
\end{aligned}
$$

which shows that anomaly lives in the axial-vector Ward identity.

However, if the external momentum acting on the amplitude is not of the axial-vector momentum but the vector momentum $\left(k_{3}-k_{1}\right)_{\mu}$. Then by using the same method, we get

$$
\begin{aligned}
\left(k_{3}-k_{1}\right)_{\mu} T_{\lambda \mu \nu}^{R, A V V} & =2 \sum_{i=0}^{2} m_{i} T_{\lambda \nu}^{P V V}\left(m_{i}\right) \\
& =-\frac{i}{2 \pi^{2}} \epsilon_{\lambda \nu \alpha \beta}\left(k_{3}-k_{1}\right)_{\alpha}\left(k_{1}-k_{2}\right)_{\beta}
\end{aligned}
$$

which indicates that anomaly lives in the vector Ward identity.

It is demonstrated from the above evaluations that one can not determine in Pauli-Villars regularization wether anomaly lives in the axial-vector Ward identity or in the vector Ward identity.

\section{CONCLUSIONS AND REMARKS}

We have investigated the triangle anomaly by using the symmetry-preserving loop regularization method developed recently in refs. 1]. As the loop regularization is realized in the initial dimension of theory without modifying the original theory, it has no $\gamma_{5}$ problem faced 
in the dimensional regularization. Also the loop regularization preserves non-Abelian gauge symmetry and satisfies a set of consistency conditions, that clearly distinguishes with the Pauli-Villars scheme. Especially, the loop regularization allows us to introduce two intrinsic mass scales without destroying any symmetries of original theory. The two intrinsic mass scales are corresponding to the characterizing energy scale $M_{c}$ and sliding energy scale $\mu_{s}$ which actually play the roles of ultra-violet and infrared cut-off energy scales respectively. It has been shown that when $k^{2} \ll \mu_{s}^{2}, m^{2} \ll M_{c}^{2} \rightarrow \infty$ with $k$ the momentum of external axialvector state and $m$ the mass of loop fermions, then both massless and massive spinor quantum gauge theories become anomaly free. It implies that the loop regularization proposed in refs. 1] can be regarded as a fully symmetry-preserving regularization method by keeping a sufficiently large IR cut-off scale $\mu_{s}$. On the other hand, it also becomes clear from loop regularization that in general the triangle anomaly appears when $m^{2}, \mu_{s}^{2} \ll k^{2} \ll M_{c}^{2} \rightarrow \infty$. The typical case of triangle anomaly occurs in the massless QED $(m=0)$ with $\mu_{s}=0$ and $M_{c} \rightarrow \infty$. Namely, the symmetry-preserving loop regularization can consistently deal with quantum anomaly and meanwhile allows an anomaly-free treatment.

Comparing loop regularization with Pauli-Villars scheme, it has been seen that the triangle anomaly in the two regularization schemes arises from different sources. In loop regularization, the direct calculation shows that, with the general conclusion of gamma trace, the triangle anomaly appears only in the axial-vector Ward identity, that should be the intrinsic property of original theory as the loop regularization is carried out without modifying the original theory. When treating the gamma trace with relation (3.11) in which the Lorentz indices of the two vector currents were classified in a group, the anomaly appears only in the two vector currents, while the Ward identity of the remaining current is still preserved. Such an anomaly can easily be shifted to the axial-vector Ward identity by a redefinition of AVV amplitudes. While in the treatment with relation (3.13) in which the gamma matrices with the Lorentz indices of a vector and the axial-vector currents are grouped to simplify the gamma trace, anomalies live in the axial-vector Ward identity and the grouped vector Ward identity while the other vector Ward identity still preserved. This anomaly can also be shifted to the axial-vector Ward identity and keep both the vector Ward identities by redefining the physical amplitude. In Pauli-Villars regularization, in all the three treatments, the vector Ward identity is preserved since the anomaly of original theory is cancelled by the heavy regulator spinors introduced in the modified lagrangian of original theory, its effect 
is equivalent to make the redefinition of AVV amplitudes in loop regularization. Although anomaly only rises in the axial-vector Ward identity, the cancellation mechanism is different in different treatments. It is then not difficult to understand why anomaly in Pauli-Villars scheme appears directly in the axial-vector Ward identity. But the anomaly is caused by the regulator spinors, which becomes unclear whether anomaly is the intrinsic property of the original theory or due to the introduction of regular fields.

In comparison with dimensional regularization, the explicit calculation shows that, in all the three treatments, the triangle anomaly in dimensional regularization receives contributions from both the $n-4$ dimensions and the original four dimensions. Consequently, both the vector and axial-vector Ward identities are violated. Nevertheless, if acting the external momentum of the axial-vector current on the AVV diagram before evaluating the integrals, the resulting triangle anomaly only depends on the extended dimensions and appears in the axial-vector Ward identity. Namely, in dimensional regularization the triangle anomaly of vector and axial-vector currents due to quantum loop corrections depends on the procedures of operation although the total anomaly when normalizing to the conserved vector current has the same standard form. Besides this ambiguity, if the acting external momentum is not operating on the axial-vector current momentum but on the vector current momentum, the same calculation gives the anomaly in the vector Ward identity. In this sense, we may state that the conventional method by acting the external momentum to reduce the triangle diagram is not a reasonable method for correctly obtaining the Ward identity anomaly.

In addition, there are also some discussions on the anomaly based on some models in which the triangle diagram with three axial-vector current couplings are considered[18]. Those results can be obtained from ours by making a redefinition for the amplitude, this is because we can always contract two $\gamma_{5}$ and reduce the AAA triangle diagram to the AVV one which has been considered above. Then one can also make a redefinition for the amplitude so that all three currents have the same amplitudes.

In conclusion, the symmetry-preserving loop regularization described in refs. [1] has been shown to be a useful regularization method for consistently dealing with anomaly of theory and providing an anomaly-free treatment of quantum field theories by keeping a sufficient large infrared cut-off scale $\mu_{s}$. A unique solution for the Ward identity anomaly of axialvector current is obtained by eliminating the ambiguity caused by the trace of gamma matrices with $\gamma_{5}$ through treating all the three currents symmetrically, which is simply 
realized by using the definition of $\gamma_{5}$ in the trace of gamma matrices.

\section{Acknowledgments}

One of authors (YLW) would like to express his thanks to David Gross for valuable discussions on anomaly-free treatment based on the loop regularization, and Roman Jackiw for stimulating and helpful discussions on the treatment of clarifying the long-standing ambiguities in the perturbative calculations of triangle anomaly. We would like to thank both Stephen Adler and Roman Jackiw for reading the manuscript and for their encouragements. This work was supported in part by the National Science Foundation of China (NSFC) under the grant 10475105, 10491306, and the Project of Knowledge Innovation Program (PKIP) of Chinese Academy of Sciences.

\section{APPENDIX A: USEFUL RELATIONS OF FEYNMAN PARAMETER INTE-} GRALS IN LOOP REGULARIZATION

Here we first introduce the definitions

$$
\begin{aligned}
Y\left(p^{2}, q^{2} ; m^{2}, \mu_{s}^{2}\right)= & \int_{0}^{1} d z \ln \left[\frac{p^{2} z(1-z)-\hat{m}^{2}}{q^{2} z(1-z)-\hat{m}^{2}}\right] \\
& +\int_{0}^{1} d z \int_{0}^{1} \frac{d \sigma}{\sigma}\left\{\exp \left\{\sigma\left[\frac{p^{2} z(1-z)-\hat{m}^{2}}{-M_{c}^{2}}\right]\right\}-\exp \left\{\sigma\left[\frac{q^{2} z(1-z)-\hat{m}^{2}}{-M_{c}^{2}}\right]\right\}\right\} \\
I_{-2,(i j)}\left(m^{2}, \mu_{s}^{2}\right)= & \frac{i}{32 \pi^{2}} \int_{0}^{1} d x_{1} \int_{0}^{x_{1}} d x_{2} \frac{x_{2}^{i}\left(x_{1}-x_{2}\right)^{j}}{-M^{2}-\hat{m}^{2}}\left[1-y_{-2}\left(\frac{\hat{m}^{2}+M^{2}}{M_{c}^{2}}\right)\right]
\end{aligned}
$$

with $p_{\mu}=\left(k_{1}-k_{2}\right)_{\mu}, \quad q_{\nu}=\left(k_{3}-k_{1}\right)_{\nu}, \hat{m}^{2}=m^{2}+\mu_{s}^{2}$ and $m$ the mass of loop fermions. It is easy to see that $I_{-2,(00)}$ is symmetric under the interchange of $p$ and $q$.

The difference between two logarithemic divergence integrals can be expressed in terms of the above integrals

$$
\begin{aligned}
& I_{0,(00)}\left(m^{2}, \mu_{s}^{2}\right) \equiv \frac{i}{16 \pi^{2}}\left\{\int_{0}^{1} d x_{1} \int_{0}^{x_{1}} d x_{2}\left[\ln \left(\frac{M_{c}^{2}}{M^{2}+\hat{m}^{2}}\right)-\gamma_{\omega}+y_{0}\left(\frac{\hat{m}^{2}+M^{2}}{M_{c}^{2}}\right)\right]\right. \\
&\left.\quad-\int_{0}^{1} d x x\left[\ln \left(\frac{M_{c}^{2}}{M_{3}^{2}+\hat{m}^{2}}\right)-\gamma_{\omega}+y_{0}\left(\frac{\hat{m}^{2}+M_{3}^{2}}{M_{c}^{2}}\right)\right]\right\} \\
&=\frac{i}{16 \pi^{2}}\left\{e^{-\hat{m}^{2} / M_{c}^{2}} \int_{0}^{1} d x_{1} \int_{0}^{x_{1}} d x_{2} e^{-M^{2} / M_{c}^{2}}-\frac{1}{2} Y\left((p+q)^{2}, q^{2} ; m^{2}, \mu_{s}^{2}\right)\right\} \\
&-q^{2} I_{-2,(10)}\left(m^{2}, \mu_{s}^{2}\right)-p^{2} I_{-2,(01)}\left(m^{2}, \mu_{s}^{2}\right)+2 \hat{m}^{2} I_{-2,(00)}\left(m^{2}, \mu_{s}^{2}\right)
\end{aligned}
$$




$$
\begin{aligned}
& I_{0,(00)}^{\prime}\left(m^{2}, \mu_{s}^{2}\right) \equiv \frac{i}{16 \pi^{2}}\left\{\int_{0}^{1} d x_{1} \int_{0}^{x_{1}} d x_{2}\left[\ln \left(\frac{M_{c}^{2}}{M^{2}+\hat{m}^{2}}\right)-\gamma_{\omega}+y_{0}\left(\frac{\hat{m}^{2}+M^{2}}{M_{c}^{2}}\right)\right]\right. \\
&\left.\quad-\int_{0}^{1} d x x\left[\ln \left(\frac{M_{c}^{2}}{M_{1}^{2}+\hat{m}^{2}}\right)-\gamma_{\omega}+y_{0}\left(\frac{\hat{m}^{2}+M_{1}^{2}}{M_{c}^{2}}\right)\right]\right\} \\
&=\frac{i}{16 \pi^{2}}\left\{e^{-\hat{m}^{2} / M_{c}^{2}} \int_{0}^{1} d x_{1} \int_{0}^{x_{1}} d x_{2} e^{-M^{2} / M_{c}^{2}}-\frac{1}{2} Y\left((p+q)^{2}, p^{2} ; m^{2}, \mu_{s}^{2}\right)\right\} \\
& \quad-q^{2} I_{-2,(10)}\left(m^{2}, \mu_{s}^{2}\right)-p^{2} I_{-2,(01)}\left(m^{2}, \mu_{s}^{2}\right)+2 \hat{m}^{2} I_{-2,(00)}\left(m^{2}, \mu_{s}^{2}\right)
\end{aligned}
$$

It should be noticed that although $I_{0,(00)}$ and $I_{0,(00)}^{\prime}$ are UV scale dependence, it is actually finite and independent of $M_{c}^{2}$ in the limit $M_{c} \rightarrow \infty$.

Using the tricks of partial integral and changing the integrating variable, one can easily get the following useful forms

$$
\begin{aligned}
& I_{-2,(01)}\left(m^{2}, \mu_{s}^{2}\right)=\frac{-i}{64 \pi^{2}} \frac{(p \cdot q) q^{2}}{p^{2} q^{2}-(p \cdot q)^{2}} \\
& \times\left\{\frac{1}{q^{2}} Y\left((p+q)^{2}, p^{2} ; m^{2}, \mu_{s}^{2}\right)+\frac{1}{p \cdot q} Y\left((p+q)^{2}, q^{2} ; m^{2}, \mu_{s}^{2}\right)\right\} \\
& +\frac{q^{2}\left[(p \cdot q)+p^{2}\right]}{2\left[p^{2} q^{2}-(p \cdot q)^{2}\right]} I_{-2,(00)}\left(m^{2}, \mu_{s}^{2}\right) \\
& I_{-2,(10)}\left(m^{2}, \mu_{s}^{2}\right)=\left.I_{-2,(01)}\left(m^{2}, \mu_{s}^{2}\right)\right|_{p \leftrightarrow q} \\
& I_{-2,(11)}\left(m^{2}, \mu_{s}^{2}\right)=\frac{(p \cdot q) q^{2}}{p^{2} q^{2}-(p \cdot q)^{2}}\left\{-\frac{i}{64 \pi^{2}} \frac{1}{q^{2}} e^{-\hat{m}^{2} / M_{c}^{2}} \int_{0}^{1} d x_{1} \int_{0}^{x_{1}} d x_{2} e^{-M^{2} / M_{c}^{2}}\right. \\
& -\frac{i}{128 \pi^{2}} \frac{\left[2 p^{4} q^{2}+3 p^{2} q^{2} p \cdot q+p^{2}(p \cdot q)^{2}\right]}{2 q^{2} p \cdot q\left[p^{2} q^{2}-(p \cdot q)^{2}\right]} Y\left((p+q)^{2}, p^{2} ; m^{2}, \mu_{s}^{2}\right) \\
& -\frac{i}{128 \pi^{2}} \frac{2 p^{2} q^{2}+3 p^{2} p \cdot q+(p \cdot q)^{2}}{2 p \cdot q\left[p^{2} q^{2}-(p \cdot q)^{2}\right]} Y\left((p+q)^{2}, q^{2} ; m^{2}, \mu_{s}^{2}\right) \\
& \left.+\left\{\frac{p^{2}\left[4(p \cdot q)^{2}+3\left(p^{2}+q^{2}\right) p \cdot q+2 p^{2} q^{2}\right]}{8 p \cdot q\left[p^{2} q^{2}-(p \cdot q)^{2}\right]}-\frac{\hat{m}^{2}}{2 q^{2}}\right\} I_{-2,(00)}\left(m^{2}, \mu_{s}^{2}\right)\right\} \\
& I_{-2,(20)}\left(m^{2}, \mu_{s}^{2}\right)=\frac{p^{2}}{p^{2} q^{2}-(p \cdot q)^{2}}\left\{-\frac{i}{64 \pi^{2}} \frac{1}{p \cdot q} e^{-\hat{m}^{2} / M_{c}^{2}} \int_{0}^{1} d x_{1} \int_{0}^{x_{1}} d x_{2} e^{-M^{2} / M_{c}^{2}}\right. \\
& -\frac{i}{128 \pi^{2}} \frac{3 p^{4} q^{2}+3 p^{4}(p \cdot q)+2 p^{2} q^{2} p \cdot q-2(p \cdot q)^{2}}{2 p^{2}\left[p^{2} q^{2}-(p \cdot q)^{2}\right]} Y\left((p+q)^{2}, p^{2} ; m^{2}, \mu_{s}^{2}\right) \\
& -\frac{i}{128 \pi^{2}} \frac{p^{2} q^{2}+p \cdot q\left[3 q^{2}+2 p \cdot q\right]}{2\left[p^{2} q^{2}-(p \cdot q)^{2}\right]} Y\left((p+q)^{2}, q^{2} ; m^{2}, \mu_{s}^{2}\right) \\
& \left.+\left\{\frac{p^{2}\left[3 q^{4}+q^{2} p^{2}+6 q^{2}(p \cdot q)+2(p \cdot q)^{2}\right]}{8\left[p^{2} q^{2}-(p \cdot q)^{2}\right]}-\frac{\hat{m}^{2}}{2}\right\} I_{-2,(00)}\left(m^{2}, \mu_{s}^{2}\right)\right\} \\
& I_{-2,(02)}\left(m^{2}, \mu_{s}^{2}\right)=\left.I_{-2,(20)}\left(m^{2}, \mu_{s}^{2}\right)\right|_{p \leftrightarrow q}
\end{aligned}
$$

With the above expressions, we can arrive at the following useful relations

$$
q^{2} I_{-2,(11)}\left(m^{2}, \mu_{s}^{2}\right)-(p \cdot q) I_{-2,(02)}\left(m^{2}, \mu_{s}^{2}\right)=\frac{-i}{128 \pi^{2}} Y\left((p+q)^{2}, p^{2} ; m^{2}, \mu_{s}^{2}\right)
$$




$$
\begin{aligned}
& +\frac{q^{2}}{2} I_{-2,(01)}\left(m^{2}, \mu_{s}^{2}\right) \\
& q^{2} I_{-2,(20)}\left(m^{2}, \mu_{s}^{2}\right)-(p \cdot q) I_{-2,(11)}\left(m^{2}, \mu_{s}^{2}\right)=\frac{-i}{64 \pi^{2}} e^{-\hat{m}^{2} / M_{c}^{2}} \int_{0}^{1} d x_{1} \int_{0}^{x_{1}} d x_{2} e^{-M^{2} / M_{c}^{2}} \\
& -\frac{\hat{m}^{2}}{2} I_{-2,(00)}\left(m^{2}, \mu_{s}^{2}\right)+\frac{p^{2}}{4} I_{-2,(01)}\left(m^{2}, \mu_{s}^{2}\right) \\
& +\frac{3 q^{2}}{4} I_{-2,(10)}\left(m^{2}, \mu_{s}^{2}\right) \\
& p^{2} I_{-2,(02)}\left(m^{2}, \mu_{s}^{2}\right)-(p \cdot q) I_{-2,(11)}\left(m^{2}, \mu_{s}^{2}\right)=\frac{-i}{64 \pi^{2}} e^{-\hat{m}^{2} / M_{c}^{2}} \int_{0}^{1} d x_{1} \int_{0}^{x_{1}} d x_{2} e^{-M^{2} / M_{c}^{2}} \\
& -\frac{\hat{m}^{2}}{2} I_{-2,(00)}\left(m^{2}, \mu_{s}^{2}\right)+\frac{q^{2}}{4} I_{-2,(10)}\left(m^{2}, \mu_{s}^{2}\right) \\
& +\frac{3 p^{2}}{4} I_{-2,(01)}\left(m^{2}, \mu_{s}^{2}\right) \\
& p^{2} I_{-2,(11)}\left(m^{2}, \mu_{s}^{2}\right)-(p \cdot q) I_{-2,(20)}\left(m^{2}, \mu_{s}^{2}\right)=\frac{-i}{128 \pi^{2}} Y\left((p+q)^{2}, q^{2} ; m^{2}, \mu_{s}^{2}\right) \\
& +\frac{p^{2}}{2} I_{-2,(10)}\left(m^{2}, \mu_{s}^{2}\right) \\
& q^{2} I_{-2,(10)}\left(m^{2}, \mu_{s}^{2}\right)-(p \cdot q) I_{-2,(01)}\left(m^{2}, \mu_{s}^{2}\right)=\frac{-i}{64 \pi^{2}} Y\left((p+q)^{2}, p^{2} ; m^{2}, \mu_{s}^{2}\right) \\
& +\frac{q^{2}}{2} I_{-2,(00)}\left(m^{2}, \mu_{s}^{2}\right) \\
& p^{2} I_{-2,(01)}\left(m^{2}, \mu_{s}^{2}\right)-(p \cdot q) I_{-2,(10)}\left(m^{2}, \mu_{s}^{2}\right)=\frac{-i}{64 \pi^{2}} Y\left((p+q)^{2}, q^{2} ; m^{2}, \mu_{s}^{2}\right) \\
& +\frac{p^{2}}{2} I_{-2,(00)}\left(m^{2}, \mu_{s}^{2}\right)
\end{aligned}
$$

\section{APPENDIX B: USEFUL RELATIONS IN PAULI-VILLARS REGULARIZA-}

\section{TION.}

$$
\begin{aligned}
\sum_{i=0}^{2} C_{i} \int \frac{d^{4} k}{(2 \pi)^{4}} \frac{m_{i}^{2}}{\left[k^{2}-M_{1 i}^{2}\right]^{3}} & =-i \sum_{i=0}^{2} C_{i} \int \frac{d^{4} k_{E}}{(2 \pi)^{4}} \frac{m_{i}^{2}}{\left[k_{E}^{2}+M_{i}^{2}\right]^{3}}=\frac{-i}{32 \pi^{2}} \sum_{i=0}^{2} C_{i} \frac{m_{i}^{2}}{M_{i}^{2}} \\
\sum_{i=0}^{2} C_{i} \int \frac{d^{4} k}{(2 \pi)^{4}} \frac{k^{2}}{\left[k^{2}-M_{1 i}^{2}\right]^{3}} & =\frac{-i}{8 \pi^{2}} \int_{0}^{1} d z\left\{\frac{\left(M_{12}^{2}-M_{10}^{2}\right)}{M_{12}^{2} z+M_{10}^{2}(1-z)}+\frac{\left(M_{12}^{2}-M_{11}^{2}\right)}{M_{12}^{2} z+M_{11}^{2}(1-z)}\right\} \\
& =\frac{-i}{8 \pi^{2}}\left\{\ln \left(\frac{M_{12}^{2}}{M_{10}^{2}}\right)+\ln \left(\frac{M_{12}^{2}}{M_{11}^{2}}\right)\right\}=\frac{i}{8 \pi^{2}} \sum_{i=0}^{2} \ln \left(\frac{M_{1 i}^{2}}{\mu^{\prime 2}}\right) \\
\sum_{i=0}^{2} C_{i} \int \frac{d^{4} k}{(2 \pi)^{4}} \frac{1}{\left[k^{2}-M_{2 i}^{2}\right]^{2}} & =\frac{i}{16 \pi^{2}} \int_{0}^{1} d z\left\{\frac{\left(M_{22}^{2}-M_{20}^{2}\right)}{M_{22}^{2} z+M_{20}^{2}(1-z)}+\frac{\left(M_{22}^{2}-M_{21}^{2}\right)}{M_{22}^{2} z+M_{21}^{2}(1-z)}\right\} \\
& =\frac{i}{16 \pi^{2}}\left\{\ln \left(\frac{M_{22}^{2}}{M_{20}^{2}}\right)+\ln \left(\frac{M_{22}^{2}}{M_{21}^{2}}\right)\right\}=\frac{-i}{16 \pi^{2}} \sum_{i=0}^{2} \ln \left(\frac{M_{2 i}^{2}}{\mu^{\prime 2}}\right)
\end{aligned}
$$




$$
\begin{aligned}
\sum_{i=0}^{2} C_{i} \int \frac{d^{4} k}{(2 \pi)^{4}} \frac{1}{\left[k^{2}-M_{3 i}^{2}\right]^{2}} & =\frac{i}{16 \pi^{2}} \int_{0}^{1} d z\left\{\frac{\left(M_{32}^{2}-M_{30}^{2}\right)}{M_{32}^{2} z+M_{30}^{2}(1-z)}+\frac{\left(M_{32}^{2}-M_{32}^{2}\right)}{M_{32}^{2} z+M_{31}^{2}(1-z)}\right\} \\
& =\frac{i}{16 \pi^{2}}\left\{\ln \left(\frac{M_{32}^{2}}{M_{30}^{2}}\right)+\ln \left(\frac{M_{32}^{2}}{M_{31}^{2}}\right)\right\}=\frac{-i}{16 \pi^{2}} \sum_{i=0}^{2} \ln \left(\frac{M_{3 i}^{2}}{\mu^{\prime 2}}\right)
\end{aligned}
$$

\section{APPENDIX C: MOMENTUM INTEGRAL IN DIMENSIONAL REGUALAR-}

\section{IZATION}

$$
\begin{aligned}
\int \frac{d^{n} k}{(2 \pi)^{n}} \frac{1}{\left(k^{2}-\Delta\right)^{3}} & =-i \int \frac{d^{n} k_{E}}{(2 \pi)^{n}} \frac{1}{\left(k_{E}^{2}+\Delta\right)^{3}} \\
& =\frac{-i}{(4 \pi)^{n / 2}} \frac{\Gamma\left(3-\frac{n}{2}\right)}{\Gamma(3)}\left(\frac{1}{\Delta}\right)^{3-\frac{n}{2}}=\frac{-i}{32 \pi^{2}} \frac{1}{\Delta} \\
\int \frac{d^{n} k}{(2 \pi)^{n}} \frac{1}{\left(k^{2}-\Delta\right)^{2}} & =i \int \frac{d^{n} k_{E}}{(2 \pi)^{n}} \frac{1}{\left(k_{E}^{2}+\Delta\right)^{2}} \\
& =\frac{i}{(4 \pi)^{n / 2}} \frac{\Gamma\left(2-\frac{n}{2}\right)}{\Gamma(2)}\left(\frac{1}{\Delta}\right)^{2-\frac{n}{2}}=\frac{i}{16 \pi^{2}}[-\ln \Delta+C] \\
\int \frac{d^{n} k}{(2 \pi)^{n}} \frac{k^{2}}{\left(k^{2}-\Delta\right)^{3}} & =\int \frac{d^{n} k}{(2 \pi)^{n}}\left[\frac{1}{\left(k^{2}-\Delta\right)^{2}}+\frac{\Delta}{\left(k^{2}-\Delta\right)^{3}}\right] \\
\int \frac{d^{n} k}{(2 \pi)^{n}} \frac{\left(k_{\perp}\right)^{2}}{\left[k^{2}-M_{1}^{2}\right]^{3}} & =\frac{-i}{32 \pi^{2}}
\end{aligned}
$$

[1] Y.L.Wu, Int. J. Mod. Phys. A18 (2003) 5363-5419, hep-th/0209021;

Y.L.Wu, Mod. Phys. Lett. A19 (2004) 2191, hep-th/0311082.

[2] D.G.Sutherland, Phys.Lett.23,384(1966); Nucl.Phys.B2,433(1967);

M.Veltman, Proc.R.Soc.London,A301,107(1967).

[3] J.S. Bell and R. Jackiw, Nuovo Cimento A 6047 (1969).

[4] S.L. Adler, Phys. Rev. 1772426 (1969).

[5] S.L. Adler and W.A. Bardeen, Phys. Rev. 1821517 (1969);

[6] W. Bardeen, Phys. Rev. 1841848 (1969).

[7] I.S. Gerstein and R. Jackiw, Phys. Rev. 1811955 (1969).

[8] S.L. Adler, R.W. Brown, T.F. Wong, and B.L. Young, Phys. Rev. D 41787 (1971). 
[9] For a Review see, e.g., S.L. Adler, hep-th/0405040, 2004, and references therein.

[10] W. Pauli and F. Villars, Rev. Mod. Phys. 21, 434 (1949); see also C. Itzykson, J.B. Zuber, QUANTUM FIELD THEORY. New York, Usa: Mcgraw-hill (1980) 705 P.(Intern. Series In Pure and Applied Physics).

[11] G.'t Hooft and M. Veltman, Nucl. Phys. B44, 189 (1972).

[12] R.Jackiw and K.Johnson, Phys.Rev. 182, 1459 (1969).

[13] K.Fujikawa, Phys.Rev.Lett.42, 1195(1979).K.Fujikawa, Phys.Rev.D 21,2848(1980).

[14] E. Witten, Nucl.Phys.B 223, 422, 433 (1983);

B. Zumino, UCB-PTH-83/16, LBL-16747, 1983;

K.C. Chou, H.Y. Guo, K. Wu, X.C. Song, Phys.Lett.B134, 67(1984);

L.Alvarez-Gaume and P.Ginsparg, Nucl.Phys.B 449,(1984);

K.C. Chou, Y.L. Wu and Y.B. Xie, Mod. Phys. Lett. A1 23(1986).

[15] Y.B. Dai and Y.L. Wu, Eur. Phys. J. C39 s1 (2004), (DOI) 10.1140/epjcd/s2004-01-001-3, hep-ph/0304075.

[16] W.Siegel, Phys.Lett.B94, 37(1980).

[17] see e.g., C.Itzykson and J.B.Zuber, Quantum Field Theory. New York : McGraw-Hill International Book Co., 1980.

[18] D.J.Gross and R.Jackiw, Phys.Rev.D 6, 477(1972) ;

D.W.McKay and B.L. Young, Phys.Rev. D 28, 1039(1983). 